\title{
Distributed Field Reconstruction in Wireless Sensor Networks Based on Hybrid Shift-Invariant Spaces
}

ausgeführt zum Zwecke der Erlangung des akademischen Grades eines

Doktors der technischen Wissenschaften

\author{
unter der Leitung von \\ Ao. Univ.-Prof. Dr. G. Matz \\ Institute of Telecommunications
}

eingereicht an der Technischen Universität Wien

Fakultät für Elektrotechnik und Informationstechnik

von

Dipl.-Ing. Günter Reise

Hirschengasse $21 / 14$

1060 Wien

Wien, im September 2011 

Die Begutachtung dieser Arbeit erfolgte durch:

1. Ao. Univ.-Prof. Dipl.-Ing. Dr. Gerald Matz Institute of Telecommunications

Technische Universität Wien

2. Carles Antón-Haro, PhD

Centre Tecnològic de Telecomunicacions de Catalunya (CTTC)

Castelldefels, Spanien 

"Das Glück des Forschers besteht nicht darin, eine Wahrheit zu besitzen, sondern die Wahrheit zu erringen.” — MAX PLANCK (1858-1947) 

The work presented in this dissertation was funded by the Austrian Science Fund (FWF) under Grants N10602 and N10606 and by the Vienna Science and Technology Fund (WWTF) under Grant ICT08-044.

This document has been typeset using $\mathrm{IET}_{\mathrm{E}} \mathrm{X}$. Numerical simulations have been carried out using MATLAB ${ }^{\circledR}$ by MathWorks ${ }^{\circledR}$. The resulting plots and figures have been enhanced and prepared using OmniGraffle Professional for Mac by The OmniGroup. 



\section{Abstract}

The interest in wireless sensor networks as solution to distributed inference problems in quite diverse monitoring applications has been steadily increasing in the last years. The basic idea is to use a large number of wirelessly connected sensor nodes, spatially distributed over the region to be monitored, that collaborate in order to collect, process, and communicate information about the phenomenon of interest. In general, wireless sensor networks are deployed in remote regions so that the sensor nodes are difficult to access. This complicates or even prevents their maintenance, e.g., recharging sensor node batteries, and makes it desirable that the wireless sensor network works with a minimum of supervision. The remote sensor node deployment and the unsupervised nature of the wireless sensor network fuel the interest in a high network lifetime, i.e., the time the network is capable to perform the desired tasks, regardless of failures of some sensor nodes. The sensor nodes are therefore required to be durable and to withstand harsh environmental conditions and the wireless sensor network has to operate at low computational complexity and with low communication overhead.

In this dissertation we are specifically concerned with the reconstruction of physical fields from irregular samples provided by the sensor nodes of a wireless sensor network. The sampling and reconstruction problem has been previously addressed in the literature under the often unrealistic assumption that the field is spatially strictly bandlimited. This assumption is too restrictive in many physical scenarios, such as for non-wave fields like electrostatic fields or diffusion fields in liquids and gases. Moreover, in the context of wireless sensor networks, it is inherently impossible to pre-process the analog field as it is done using anti-aliasing filters in conventional temporal sampling applications.

We use the theory of shift-invariant spaces to model physical fields and to develop a scheme based on this model for sampling and reconstruction of time-varying, non-bandlimited physical fields. Our field reconstruction is based on the least-squares estimation of the model coefficients. As generator functions that span the shift-invariant spaces we use such of compact support, in particular Basis-splines, that provide excellent interpolation properties and allow us to reduce the computational complexity of the reconstruction, leading to a complexity that scales linearly with the number of sensor nodes. Moreover, the use of compactly supported generator functions admits the partitioning of the sensor nodes into clusters, thereby splitting up the field reconstruction into smaller problems that can be independently solved by the sensor node clusters. Within the overlapping subregions corresponding to the clusters, the estimates for the field are averaged for better reconstruction quality. We extend and generalize shift-invariant spaces by using different generator functions within the clusters, thereby introducing hybrid shift-invariant spaces. These allow better adaption of the field model to local smoothness properties of the field to be reconstructed. For time-varying fields, we show that the use 
of iterative solvers initialized with the solution from the previous time-step yields large savings in the computational efforts necessary for the reconstruction. We furthermore analyze the impact of sensor localization errors on the mean square error of the reconstructed field. Numerical simulations illustrate the performance of our proposed field reconstruction scheme and show that it is less sensitive to sensor localization errors than bandlimited reconstruction while providing a significant reduction in computational complexity.

In the context of our field reconstruction scheme, we also introduce an amplify-and-forward scheme for the transmission of sensor node measurements to the fusion center (or cluster head). We derive the optimal sensor node transmit power allocation for minimal mean square error and minimal transmit sum power respectively. In both cases the power allocation problem is convex and can be solved numerically in an efficient manner. For the special case of critical sampling we derive closed-form solutions for these convex problems. In numerical simulations we compare the performance of the proposed power allocation schemes with uniform power allocation.

In summary, we present a robust wireless sensor network architecture for sampling and reconstruction of non-bandlimited time-varying fields that provides excellent reconstruction quality with low computational complexity and low communication overhead. The corresponding optimal transmit power allocation scheme takes into account the sensor node positions and thereby preserves a stable reconstruction. 


\section{Kurzfassung}

Das Interesse an drahtlosen Sensornetzen zur Lösung von verteilten Inferenzproblemen in verschiedenen Überwachungsanwendungen stieg in den letzten Jahren kontinuierlich an. Die grundsätzliche Idee liegt in der Verwendung einer großen Anzahl von drahtlos verbundenen Sensorknoten, die räumlich in einem zu überwachenden Gebiet verteilt sind. Die Sensorknoten kooperieren bei der Datensammlung, -verarbeitung und -übertragung, um Informationen über das Phänomen von Interesse zu erhalten. Im Allgemeinen werden drahtlose Sensornetze in abgelegenen Gebieten verwendet. Das erschwert oder verhindert die Wartung der Sensorknoten, z.B. das Laden der Akkumulatoren, und macht es wünschenswert, drahtlose Sensornetze mit einem Minimum an Überwachung und Betreuung betreiben zu können. Die Verwendung in entlegenen Gebieten und der unbeaufsichtigte Betrieb von drahtlosen Sensornetzen motivieren das Interesse an einer langen Betriebsdauer, wobei die Betriebsdauer jene Zeit ist, die das Sensornetz seine Aufgabe erfüllt, trotz eventuellen Ausfalls einzelner Sensoren. Die Sensorknoten müssen daher schwierigen Umweltbedingungen widerstehen und das Sensornetz muss mit geringem Berechnungs- und Kommunikationsaufwand betrieben werden können.

In dieser Dissertation beschäftigen wir uns mit der Rekonstruktion von physikalischen Feldern aus unregelmäßigen Abtastwerten, die von Sensorknoten eines drahtlosen Sensornetzes bereitgestellt werden. Das Abtastungs- und Rekonstruktionsproblem wurde in der Literatur unter der oft unrealistischen Annahme behandelt, dass das Feld räumlich streng bandbegrenzt ist. Diese Annahme ist für viele physikalischen Szenarien zu restriktiv, z.B. für Nicht-Wellenfelder wie elektrostatische Felder oder Diffusionsfelder in Flüssigkeiten und Gasen. Darüber hinaus ist bei drahtlosen Sensornetzen die Vorbearbeitung des analogen Feldes mittels eines Anti-Aliasing Filters, wie es bei herkömmlichen zeitlichen Abtastungsproblemen gemacht wird, nicht möglich.

Wir verwenden verschiebungsinvariante Räume als Modell für physikalische Felder. Basierend auf diesem Modell entwickeln wir ein System für die Abtastung- und Rekonstruktion von zeitvarianten, nicht bandbegrenzten Feldern aus unregelmäßigen Abtastwerten. Unsere Rekonstruktion basiert auf einer Least-SquaresSchätzung der Modellkoeffizienten. Als Generatorfunktionen für die verschiebungsinvariante Räume verwenden wir solche mit kompaktem Träger, insbesondere verwenden wir B-splines. Diese bieten exzellente Interpolationseigenschaften und erlauben eine Rekonstruktion des Feldes mit reduziertem Berechnungsaufwand, d.h. mit einer Komplexität, die linear mit der Anzahl der Knoten zunimmt. Zudem erlaubt die Verwendung von Generatorfunktionen mit kompaktem Träger die Partitionierung der Sensorknoten in Cluster und die Unterteilung der Rekonstruktion in kleinere Probleme, die voneinander unabhängig innerhalb der einzelnen Cluster gelöst werden können. Innerhalb von zu Clustern gehörenden überlappenden Teilgebieten können Schätzwerte für das Feld für eine bessere Rekonstruktionsqualität gemittelt werden. Wir erweitern und verallgemeinern verschiebungsinvariante Räume durch die Verwendung von unterschiedlichen Generatorfunktionen innerhalb der 
einzelnen Cluster und führen damit hybride verschiebungsinvariante Räume ein. Diese erlauben eine bessere Anpassung des Feldmodells an die lokalen Eigenschaften des zu rekonstruierenden Feldes. Für zeitvariante Felder zeigen wir, dass die Verwendung von iterativen Lösungsverfahren, die mit der Lösung aus dem vorhergehenden Zeitschritt initialisiert werden, große Einsparungen beim Berechnungsaufwand erlauben. Wir untersuchen außerdem die Auswirkungen von Lokalisierungsfehlern der Sensorknoten auf den mittleren quadratischen Fehler des rekonstruierten Feldes. Numerische Simulationen veranschaulichen die Leistungsfähigkeit unseres Rekonstruktionssystems und zeigen, dass es gegenüber Fehlern bei der Lokalisierung von Sensorknoten weniger empfindlich ist als bandbegrenzte Rekonstruktionsverfahren und den Berechnungsaufwand für die Rekonstruktion erheblich reduziert.

Für das von uns vorgestellte Rekonstruktionssystem führen wir ein auf Amplify-and-Forward basierendes Verfahren für die Übertragung der Messwerte der Sensorknoten zum Fusion Center (oder Cluster Head) ein. Wir leiten die optimale Zuteilung der Sendeleistung an die Sensorknoten für den minimalen quadratischen Fehler bzw. die minimale Gesamtsendeleistung her. In beiden Fällen zeigt sich, dass das optimale Zuteilungsproblem ein konvexes Optimierungsproblem darstellt, das sich numerisch effizient lösen lässt. Für den Spezialfall von kritischer Abtastung leiten wir geschlossene Lösungen für die konvexen Optimierungsprobleme her. Mittels numerischer Simulationen vergleichen wir die Leistungsfähigkeit der hergeleiteten $\mathrm{Zu}-$ teilungsverfahren miteinander sowie mit einer gleichförmigen Zuteilung der Sendeleistung an die Sensorknoten.

Zusammengefasst präsentieren wir eine robuste drahtlose Sensornetzarchitektur für die Abtastung und Rekonstruktion von nicht bandbegrenzten, zeitvarianten Feldern, das ausgezeichnete Rekonstruktionsqualität bei gleichzeitiger geringer Berechnungskomplexität und geringem Kommunikationsaufwand erlaubt. Die entsprechende optimale Zuteilung der Sendeleistung an die Sensorknoten nimmt Rücksicht auf die Positionen der Sensorknoten und bewahrt dadurch eine stabile Rekonstruktion. 


\section{Acknowledgments}

I would like to thank the people who made the last years an amazing and unforgettable experience. My supervisor, Gerald Matz, deserves particular attention and many thanks for his continuous support. He provided me with a lot of freedom in my research and our numerous creative discussions have been essential for my professional, intellectual, and personal development. I have benefited tremendously from his efforts and dedication which helped me to reach several milestones in my academic career.

Furthermore, I want to thank Carles Antón-Haro and Javier Matamoros Morcillo for the support of my work during my visit at the Centre Tecnològic de Telecomunicacions de Catalunya (CTTC) in Castelldefels, Spain. I thank them very much for the friendly and generous reception as well as for the kind working environment that allowed me to make giant leaps in my work in little time. My gratitude especially goes to Carles Antón-Haro for agreeing to act as a referee and for contributing his broad perspective in refining the ideas in this dissertation.

I am grateful to all my former and current colleagues at the Institute of Telecommunications of the Vienna University of Technology, in particular the people of my research group, for providing a truly amicable and enriching environment and for helping me to solve occasional technical and mathematical problems. They made my days at the institute interesting, exciting, and enjoyable.

Moreover I want to thank my friends outside of the University environment for keeping me in the mood for my work and for being there to help me whenever I needed them.

Last, but far from least, I would like to express my deep gratitude to my parents and siblings. Their encouragement, support, and confidence in me through all the years of my life have meant more than I could ever express. 



\section{Contents}

Abstract

Kurzfassung

Acknowledgments xiii

1 Introduction 1

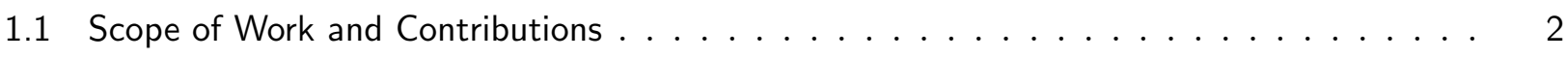

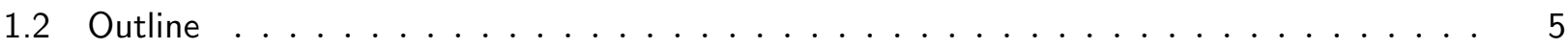

2 Preliminaries $\quad 7$

2.1 Wireless Sensor Networks $\ldots \ldots \ldots \ldots . \ldots \ldots \ldots$

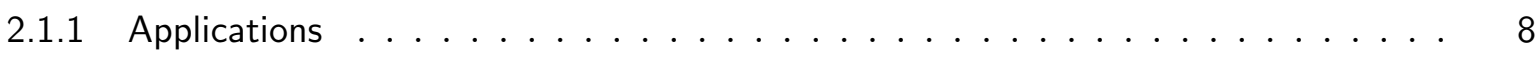

2.1 .2 Sensor Hardware . . . . . . . . . . . . . . . . . . . . . . . . 8

2.1.3 Sensor Node Deployment and Network Topology . . . . . . . . . . . . . . 10

2.1 .4 Sensor Node Clustering . . . . . . . . . . . . . . . . . . . . . . . . . 11

2.1 .5 Sensor Node Localization . . . . . . . . . . . . . . . . . . . . . . 12

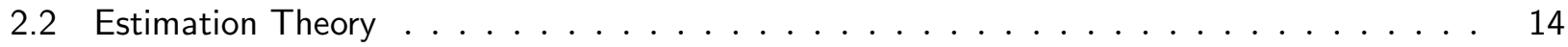

2.2 .1 Classical Estimation . . . . . . . . . . . . . . . . . . . . 14

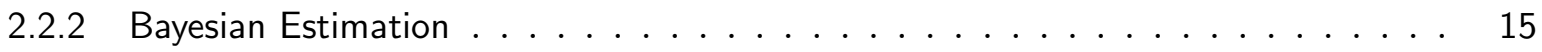

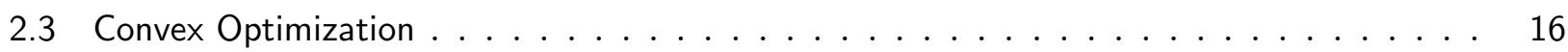


3 Distributed Field Reconstruction 19

3.1 Background and State of the Art . . . . . . . . . . . . . . . . . 20

3.2 System Model . . . . . . . . . . . . . . . . . . . . 21

3.2.1 Wireless Sensor Network Architecture . . . . . . . . . . . . . . . . . 21

3.2 .2 Measurement Model . . . . . . . . . . . . . . . . . . . . 22

3.3 Field Model - Shift-Invariant Spaces . . . . . . . . . . . . . . . . . . . . . . 23

3.3.1 Conventional Shift-Invariant Spaces . . . . . . . . . . . . . . . . . . . . 23

3.3.2 Compactly Supported Generator Functions . . . . . . . . . . . . . . . . . . . . . 24

3.3.3 Hybrid Shift-Invariant Spaces . . . . . . . . . . . . . . . . . . . 25

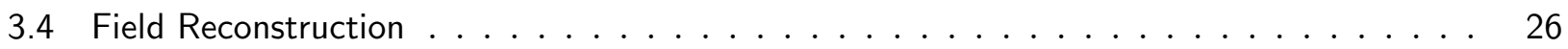

3.4.1 Centralized Case - Global Reconstruction . . . . . . . . . . . . . . . . . 26

3.4 .2 Clustered Reconstruction . . . . . . . . . . . . . . . . . . . . . . 27

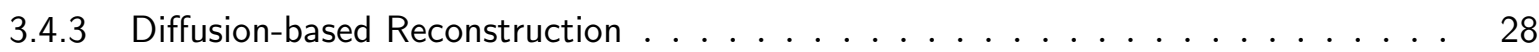

3.5 Implementation of Reconstruction Algorithm . . . . . . . . . . . . . . . . 29

3.5 .1 Matrix-Vector Formulation . . . . . . . . . . . . . . . . . . . . . 29

3.5.2 Efficient Solvers for the Sparse LS Problem . . . . . . . . . . . . . . . 30

3.5.3 Algorithm Summary and Complexity . . . . . . . . . . . . . . . . . . . 31

3.6 Sensor Node Placement . . . . . . . . . . . . . . . . . . . . . . . . . 31

3.6.1 Sensor Node Deployment . . . . . . . . . . . . . . . . . . 31

3.6 .2 Stable Sets of Sampling . . . . . . . . . . . . . . . . . . . 33

3.6.3 Selective Dimension Reduction . . . . . . . . . . . . . . . . . . . 34

3.6 .4 Sensor Node Localization Error . . . . . . . . . . . . . . . . . . . . . . . . . 34

3.7 Numerical Simulations . . . . . . . . . . . . . . . . . . . 36

3.7.1 Comparison of Bandlimited Reconstruction with Reconstruction using B-Splines . . 37

3.7 .2 Averaging of Coefficients . . . . . . . . . . . . . . . . . . 40

3.7.3 Reconstruction of Time-varying Fields . . . . . . . . . . . . . . . . . . . 43

3.7.4 Influence of Sensor Node Localization Error . . . . . . . . . . . . . . . . . . . . 44

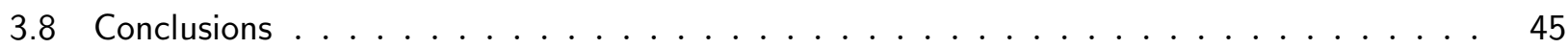

3.A Proofs .............................. 46

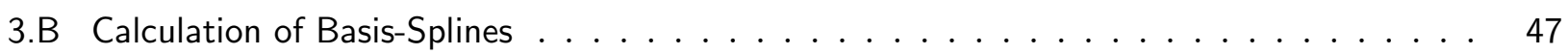

3.B.1 Calculation Using Convolution . . . . . . . . . . . . . . . . 47

3.B.2 Cox-de Boor Algorithm . . . . . . . . . . . . . . . . . . 48

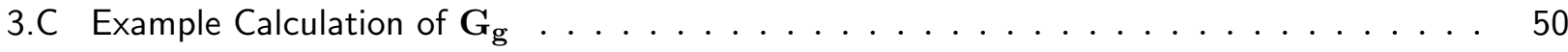


4 Optimal Power Allocation $\quad 53$

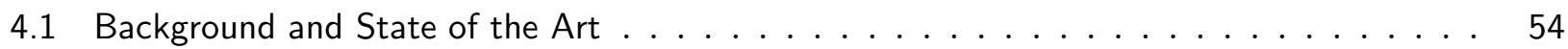

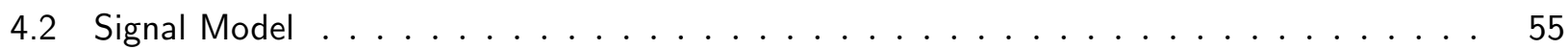

4.3 Field Reconstruction and Reconstruction Performance . . . . . . . . . . . . 56

4.4 Optimal Power Allocation: Minimization of Mean Square Error . . . . . . . . . . . . . . 58

4.4 .1 General Case . . . . . . . . . . . . . . . . . . . . . . 58

4.4 .2 Critical Sampling . . . . . . . . . . . . . . . . . . . . . 58

4.5 Optimal Power Allocation: Minimization of Transmit Sum Power . . . . . . . . . . . . 60

4.5 .1 General Case . . . . . . . . . . . . . . . . . . . . . . . . 60

4.5 .2 Critical Sampling . . . . . . . . . . . . . . . . . . . 60

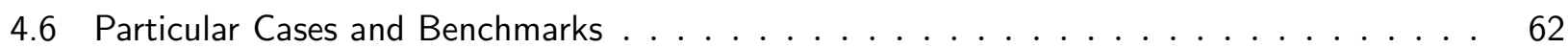

4.6 .1 Uniform Power Allocation . . . . . . . . . . . . . . . . . . . . 62

4.6 .2 Uniform Mean Square Error (MSE) Target . . . . . . . . . . . . . . . . . . 62

4.6.3 Gaussian Channels and Identical Noise Variances . . . . . . . . . . . . . . . . 63

4.7 Numerical Simulations . . . . . . . . . . . . . . . . . . . . . . . 63

4.7.1 Minimization of the Mean Square Error . . . . . . . . . . . . . . . . . . . 64

4.7 .2 Minimization of Transmit Power . . . . . . . . . . . . . . . . . . 67

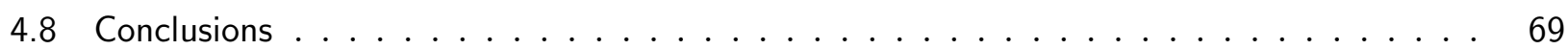

4. A Optimization Problem Revisited . . . . . . . . . . . . . . . . . . . . . 70

5 Conclusions and Outlook 71

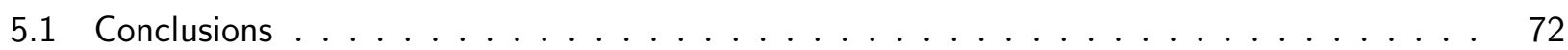

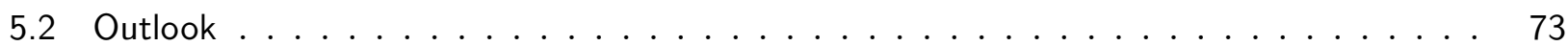

$\begin{array}{ll}\text { Acronyms } & 77\end{array}$

$\begin{array}{ll}\text { Notation } & 79\end{array}$

Bibliography 83 


\section{1 \\ Introduction}

$\mathrm{T}$

HE interest in Wireless Sensor Networks (WSNs) has been steadily increasing in recent years since they provide a solution to distributed inference problems in diverse application areas such as environmental monitoring, safety and health, security and surveillance, and the military sector. A WSN consists of many small devices, called sensor nodes. The basic idea is to use wirelessly connected sensor nodes that are spatially distributed over the region to be monitored, in order to collect, process, and communicate information about a phenomenon of interest.

Sensor nodes are typically deployed in remote areas. This complicates or even prevents maintenance tasks and makes it desirable that the WSN works with a minimum of supervision. The sensor nodes are therefore required to be durable so that they can withstand harsh environmental conditions.

Building WSNs became possible through fundamental advances in several enabling technologies. Miniaturization of hardware allowed to build smaller devices and to include further and more complex functionalities into the hardware components, making microcontrollers, Central Processing Units (CPUs), and memory, but also radio equipment more powerful and their size suitable for usage in sensor nodes of WSNs. Downsizing of the hardware components and the resulting reduced chip sizes are accompanied by reduced hardware costs and a large decrease of the hardware's power consumption. While the former are necessary to produce large numbers of inexpensive sensor nodes, the latter is crucial for reasonable sensor node and network lifetime. Technological advances resulted in small-sized, high-capacity batteries for reliable and enduring power supply that can provide small amounts of current while having a negligible self-discharge rate.

There is a great deal of requirements for protocols and algorithms used in WSNs. They need to provide scalability to allow small-scale as well as large-scale deployment of sensor nodes. In case of many sensor nodes, their positions can hardly be engineered or predetermined so that the sensor placement will in the extreme case be completely random which requires self-organization capabilities. Moreover, communication protocols and computation algorithms used in WSNs need to be flexible and able to cope with communication errors or failure of one or several sensor nodes while at the same time operating at modest computational complexity and low communication overhead. 


\subsection{Scope of Work and Contributions}

In this dissertation our focus lies on algorithms for two specific problems in WSNs. In the first part we are specifically concerned with the reconstruction of non-bandlimited physical fields from irregular samples provided by the sensor nodes of a WSN. For this application the sensor network needs to perform data acquisition (i.e., sampling of the field), data transmission, and field reconstruction (either distributed or centralized). Our focus is on the actual field reconstruction and we will thus assume simple models for the data acquisition and transmission stages.

The sampling and reconstruction problem has been previously addressed in the literature under the often unrealistic assumption that the field is spatially strictly bandlimited. In conventional temporal sampling applications, sufficient bandlimitation can be ensured by preceding the sampling with an analog anti-aliasing filter. In the context of WSN this is inherently impossible since the analog field cannot be accessed or pre-processed. At the same time, the assumption of a bandlimited field is too restrictive for many physical scenarios, e.g., electrostatic fields, gravitation fields, or diffusion fields in liquids and gases. In order to use bandlimited reconstruction with non-bandlimited physical fields, significant oversampling is required to achieve small reconstruction errors.

We pursue a new approach for the problem of sampling and reconstruction of non-bandlimited physical fields. We build on the theory and algorithms that have been developed for shift-invariant spaces and provide models for clustered sampling and reconstruction of smooth fields without the requirement of strict spatial band limitation. Our approach exploits the locality of shift-invariant spaces in combination with generator functions of compact support where we will in particular use Basis-splines (B-splines). This allows a flexible partitioning of the sensor nodes into clusters and the distribution of the field reconstruction task among these clusters, resulting in a robust and failsafe field reconstruction scheme. As a result, our approach is highly local in the sense that reconstructing the field at a certain position requires only samples from a small spatial neighborhood which leads to vast reductions in computational and communication complexity of field reconstruction. These reductions lead to energy savings and in turn to extended lifetime of the WSN. The results of the first part of the dissertation have in parts been previously published in [1-4].

In the second part of this dissertation we cover the problem of power allocation in WSNs. The general goal of power allocation is to optimally assign transmit powers to the sensor nodes of the network. The power allocation problem has been extensively investigated for linear scalar models in the context of distributed estimation in WSNs. Here, we consider the linear matrix-vector model used for our field reconstruction scheme introduced in the first part of the dissertation, hence also taking into account the sensor node positions. We pose the problems of optimal power allocation as convex optimization problems that can be solved numerically in the general case and in closed-form for the special case of critical sampling. Here, optimality can be understood with regard to two different parameters. Either the mean square estimation error is minimized subject to a transmit sum-power constraint or the transmit sum power is minimized subject to an estimation error target. The contributions of the second part of the dissertation are partly based on our previous work [5-7].

Our contributions can be summarized as follows. 


\section{Field Reconstruction in Wireless Sensor Networks}

- Field reconstruction scheme based on shift-invariant spaces: We start the dissertation by introducing a field reconstruction scheme for WSN based on shift-invariant spaces. The scheme is based on the work of Aldroubi and Gröchenig [8] as well as Gröchenig and Schwab [9]. We modify and extend their work to make it applicable for the reconstruction of Two-Dimensional (2-D) fields. Appropriately choosing network architecture and generator functions allows the scheme to be used for distributed reconstruction of non-bandlimited physical fields, which is in contrast to existing approaches.

- Generator functions of compact support: The presented field reconstruction scheme can be used with arbitrarily chosen generator functions. In particular, we propose the use of generator functions of compact support, which allows us to partition the reconstruction task among groups of sensor nodes. An especially useful class of compactly supported generator functions are B-splines, i.e., sufficiently smooth piecewise polynomial functions. B-splines are simple to construct, easy and accurate to evaluate, and show excellent properties for interpolation tasks.

- Flexible partitioning and clustering of the reconstruction task: When using generator functions of compact support, it is possible to partition the region over which the physical field is to be reconstructed into subregions and to simultaneously partition the set of sensor nodes into corresponding clusters. We show how the clusters can then reconstruct the associated subregions independently of each other, thereby reducing the overall communication needed between sensor nodes and the computational efforts necessary for reconstruction.

- Hybrid shift-invariant spaces: We extend the concept of shift-invariant spaces by allowing the use of different generator functions in different subregions. We term the resulting spaces hybrid shift-invariant spaces in contrast to conventional shift-invariant spaces. A special case uses B-splines of different order in every cluster. In this way the reconstructed field can be better adapted to local smoothness properties of the physical field.

- Reconstruction using direct and iterative solvers: The reconstruction algorithm consists of solving a linear system of equations which can be solved using either direct or iterative solvers. For time-varying fields we show that using an iterative solver is advantageous because a large part of the computational complexity can be saved by using the solution from the previous time instant to initialize the iterative solver. Then, a few iterations are sufficient, except for the case of large temporal deviations from the previous field values.

- Averaging of estimates between clusters: Partitioning of the field region and clustering of the sensor nodes of the WSN in general leads to overlapping subregions and sensor nodes being members of two or more clusters. Within overlapping subregions, the field estimates can be averaged. This leads to better estimates and augments the quality of the reconstructed field.

- Influence of sensor localization errors: The proposed field reconstruction scheme requires the knowledge of the sensor node positions. Inaccurate knowledge of these positions, e.g., because of limited 
capabilities of the localization system used by the sensor nodes, will degrade the field reconstruction quality. We analytically derive expressions that show the influence of sensor localization errors on the field reconstruction quality and we analyze the different effects of these errors for different orders of B-splines.

- Numerical performance analysis: To demonstrate the effectiveness of our reconstruction scheme, we compare the reconstruction quality achieved with the bandlimited reconstruction and reconstruction using B-splines for the example of diffusion fields for the unclustered as well as the clustered case. We compare the impact of different sensor deployment strategies and sensor node measurement noise levels on the reconstruction error. We show the capabilities of the proposed reconstruction scheme for time-variant fields, proof the efficiency of averaging of field estimates, and evaluate the influence of sensor localization errors on the reconstruction quality.

\section{Power Allocation in Wireless Sensor Networks}

- Field reconstruction performance: To evaluate the performance of the field reconstruction scheme we augment it with a transmission model and analytically derive the MSE of the reconstructed field in dependence of the factors influencing the reconstruction, such as the sensor node positions, the sensor node measurements, and the channel gains.

- Optimal power allocation for minimal MSE: We derive a power allocation scheme with the goal of minimizing the MSE of the reconstructed field under the side constraint of limited transmit sum power. The optimization problem is shown to be a variation of the well-known A-optimal convex problem [10] and can therefore be solved numerically in an efficient manner. We particularize the results for the optimal power allocation for minimal MSE to the special case of critical sampling. In that particular case it is possible to calculate a closed-form solution. The optimal transmit powers can therefore be determined directly without the need to perform a numerical optimization which results in a large complexity reduction.

- Optimal power allocation for minimal transmit sum power: With the goal of minimizing the transmit sum-power we derive a power allocation scheme under the side constraint of a limited MSE allowance for the reconstructed field. The optimization problem is again convex, allowing us to solve it numerically in an efficient manner. For the optimal power allocation for minimal transmit sum power, a particularization to the special case of critical sampling as well allows the calculation of a closed-form solution. Avoiding the numerical optimization and instead directly calculating the transmit powers leads to significant complexity savings.

- Uniform power allocation and power allocation for particular cases: As a benchmark, we compare our power allocation schemes with the straight-forward solution of uniform power allocation. We particularize the results for several special parameter sets, thereby gaining insight into the allocation mechanisms. 
- Gaussian and Rayleigh-fading channels: For the channel coefficients influencing the power allocation to the sensor nodes we assume two different channel models. First, we consider Gaussian channels between the sensor nodes and the fusion center. Second, we consider Rayleigh-fading channels. The performance under both channel models is illustrated by numerical simulations.

- Numerical performance analysis: We provide a comparison of the derived power allocation schemes, showing their influence on the reconstruction quality for Gaussian channels and Rayleigh-fading channels, respectively.

\subsection{Outline}

We next give a short outline of the structure of the rest of this dissertation.

- In Chapter 2 we review a number of basic concepts and mathematical tools which will be used in the subsequent chapters of this dissertation. We give an overview of the multitude of applications of WSNs and a brief survey of the network topologies and communication strategies used in WSNs. We continue with a short introduction to estimation theory and the theory of convex optimization.

- In Chapter 3 we introduce a novel field reconstruction scheme based on shift-invariant spaces. We use B-splines as generator functions to construct a model for non-bandlimited fields and introduce a reconstruction algorithm using nonuniform samples (taken by sensor nodes of a WSN) for the reconstruction of the field. We present centralized as well as distributed reconstruction approaches and moreover introduce an iterative reconstruction algorithm that allows time-variant fields to be reconstructed at low complexity.

- In Chapter 4 we take a close look at the power allocation problem in WSN. Specifically, for the field reconstruction scheme introduced in Chapter 3, we present optimal power allocation strategies for minimum MSE and minimum transmit sum power, respectively. We compare the optimal strategies with the optimal strategy for the special case of critical sampling and with uniform power allocation and investigate which factors influence the power allocation schemes.

- In Chapter 5, finally, we summarize the main contributions of this dissertation, draw some conclusions, and point out open issues and extensions for further research. 


\section{2 \\ Preliminaries}

$\mathrm{I}^{\mathrm{N}}$

$\mathrm{N}$ this Chapter we will review a number of basic concepts and mathematical tools which will be used in the remainder of this dissertation. We start with an introduction to Wireless Sensor Networks (WSNs), giving an overview of the multitude of applications and the hardware used in sensor nodes followed by a brief survey of the network topologies and communication strategies for WSNs. We continue with a short introduction to estimation theory, shift-invariant spaces, Basis-splines, and the theory of convex optimization, which we will use for the field reconstruction scheme presented in Chapter 3 and for the power allocation schemes presented in Chapter 4.

\subsection{Wireless Sensor Networks}

WSNs consist of a large number of small devices, called sensor nodes, that collaborate with the goal to fulfill a certain task, e.g., a monitoring task. Generally, WSNs are deployed in remote regions so that the sensor nodes operate in areas that are difficult to access and energy recharge becomes impossible. This, together with size constraints, poses severe restrictions on energy consumption, processing power and communication capacity [11].

WSNs are similar to mobile ad hoc networks in many ways which makes it possible to reuse existing concepts and solutions for ad hoc networks, e.g., self-organization techniques. However, there are some principal differences between the two. While mobile ad hoc networks are set up to meet a communication need and are designed to improve performance metrics such as throughput and delay, WSNs are tailored for specific applications, probably on a larger scale, with the major design goal of power conservation for improved network lifetime [12]. In the following section we will give an overview of the different application areas of WSNs, including some examples which will clarify the differences between WSNs and ad hoc networks. 


\subsubsection{Applications}

Based on sensor nodes with different sensing, computation, and communication capabilities, WSNs have a wide range of applications. In the following we will list and briefly describe main application areas. Note that the classification of the applications presented here is not unique and non-exhaustive. For a detailed overview and real-world application examples, see [11-13] and the references therein.

- Environmental monitoring applications are as diverse as nature itself. WSNs could be used to monitor environmental conditions, e.g., the weather, affecting flora and fauna to predict/detect incidents like floods, forest fires, or severe environmental pollution. Sensor networks are moreover used to track the movements of animals. On a larger scale, it is possible to monitor the Earth for earthquake and tsunami warning applications.

- Safety and health applications: WSNs are able to work even if existing infrastructure has been destroyed. They can be deployed for disaster relief and for tracking of and communication among rescue units. In standard health services, tele-monitoring of patient's physiological data, tracking and monitoring of hospital personnel and patients as well as drug administration are typical applications.

- Security and surveillance applications: WSNs can be used for detection and monitoring of equipment and facilities to prevent intrusion, theft, or damage.

- Military applications: The advantage of WSNs for military use is their robustness against hostile actions, i.e., destruction of (some) sensor nodes does not affect the functionality of the sensor network. Military use of WSNs comprises the monitoring of forces, equipment, and ammunition, the reconnaissance and surveillance of the battlefield and the detection of nuclear, biological, and chemical warfare agents.

- Other applications of WSNs could be environmental control in buildings, i.e., the control of air condition and heating, control of home appliances, and vehicle tracking. WSNs can also be used in logistics, e.g., for packet tracking.

\subsubsection{Sensor Hardware}

The design and development of low-cost and low-power WSNs was enabled by advances in hardware miniaturization, wireless communication, and digital electronics. A WSN consists of a large number of (usually identical) small sensor nodes that can sense their environment, process data, and communicate with neighboring sensor nodes. The sensor nodes have to work autonomously and adapt to their environment, so that it is possible to leave them unattended during operation [13]. The typical hardware components of a sensor node, illustrated in Figure 2.1, are as follows [13,14].

- The sensing unit provides the sensor node with the capability to gather information about the physical world. The possible range of sensors is vast. They measure a physical quantity at the sensor node position and deliver an analog signal which is then converted into digital data that is handed over to the processing unit. 


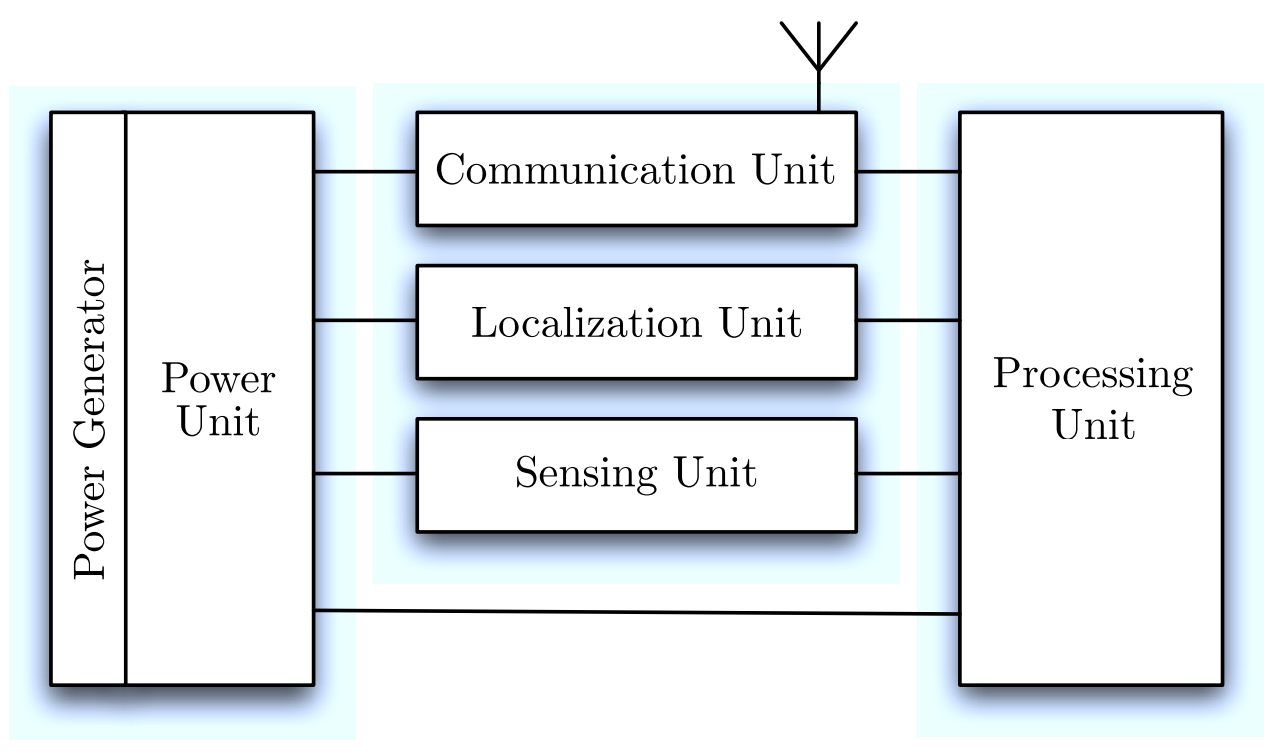

Figure 2.1: Hardware components of a sensor node

- The processing unit is the computational heart of the sensor node. It may consist of a microcontroller, a Field Programmable Gate Array (FPGA), an Application Specific Integrated Circuit (ASIC), or a Central Processing Unit (CPU) and memory. It controls the other components of the sensor node to perform the sensing operation, process the captured data, and collaborate with other sensor nodes through wireless communication.

- The communication unit provides the wireless interface for the communication between sensor nodes. For the communication, well known technologies, e.g., spread-spectrum communications or Ultra Wide Band (UWB) can be used. Most of the WSNs use radio waves as communication media, but for certain applications other means of communication can be used, e.g., optical communication or ultrasound.

- The power unit supplies the sensor node with the necessary energy. Usually the power unit is a battery, but other energy sources are also possible. The battery has to provide high capacity at a small weight, small size, and low price. Moreover, the battery's self-discharge should be low. The power unit may be complemented by a power generator, e.g., solar cells, to recharge the battery and extend sensor node and network lifetime.

- The localization system determines the position of the sensor node, which is necessary in most sensor network applications. See Section 2.1.5 for an overview of sensor node localization methods.

- A mobilizer (not shown in Figure 2.1) may be necessary for some applications to move the sensor node.

Depending on the application, further hardware units are possible. The most important concern for a WSN is the energy consumption of its sensor nodes. For most applications the WSN is inaccessible or it is not feasible to replace the power unit of the sensor nodes. In order to extend the network lifetime, which is the total time for which the WSN is operational, all components of the sensor nodes and the algorithms used in the WSN have 


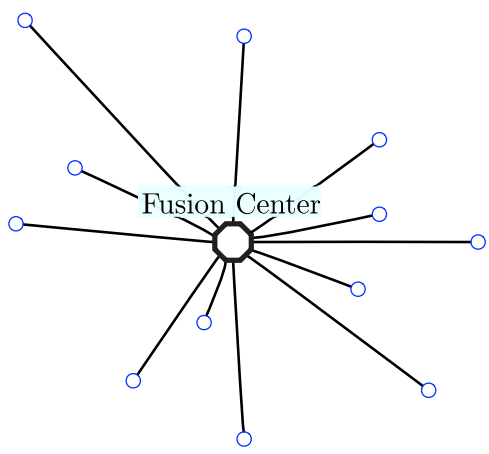

(a)

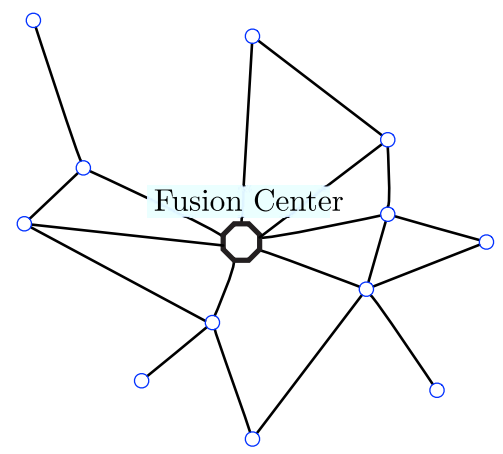

(b)

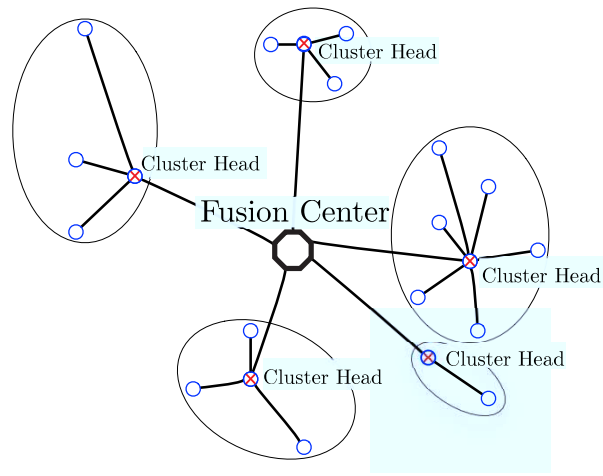

(c)

Figure 2.2: Network Topologies: (a) star network; (b) mesh-network (multi-hop); (c) hierarchical/cluster-tree network

to be designed with the goal of a low power consumption [12]. Depending on the network topology, a trade-off between computation and communication efforts has to be found to achieve energy efficiency. Since sensor nodes are deployed once and are usually not recollected for reuse after their battery has drained, the production costs of such sensor nodes have to be low in order to allow a WSN to be used reasonably in an application.

\subsubsection{Sensor Node Deployment and Network Topology}

The deployment of the sensor nodes in a WSN is crucial in terms of both communication and sensing. They can either be placed one by one in the area of interest or they can be deployed in mass, e.g., by dropping them from an airplane. In the latter case the position of each sensor will result random, which has to be borne in mind for the design and development of WSN algorithms. In most cases, however, the application will impose constraints on the deployment method for the sensor nodes.

As we will discuss in Chapter 3, the field reconstruction quality is not only influenced by the number of sensor nodes used, as can be expected using noisy measurements, but also by their placement. Sensor node deployment and placement moreover influences the communication between sensor nodes and the fusion center. The condition of the terrain, the basics of radio communication, and the sensor nodes' restricted transmit power lead to a limitation on the feasible distance between a sender and a receiver. This results in a topology of the network, which, in the sense of graph theory, can be defined as the sensor nodes that are available for communication, i.e., the vertices, and the wireless links between these nodes used for communication, i.e., the edges [12].

If single-hop communication between the sensor nodes and the fusion center is possible, we obtain a star network (see Figure 2.2(a)). However, in case the limited communication distance prevents the simple and direct communication between source and destination, other sensor nodes can be used as relays and data can be transmitted to the destination via multiple hops. This can happen especially when the WSN covers a larger area or operates in difficult radio environments with strong attenuation or large obstacles. Multi-hop communication in addition to providing connectivity, can help to save energy for large distances between source and destination 
but wastes energy for small distances $[13,14]$. Therefore, great care should be taken when applying multihopping with the goal of improved energy efficiency. In addition to the energy efficiency, routing techniques for WSNs [15] have to take into account changes of the topology during operation of the WSNs which may happen due to node failures, energy shortage, or changes in the surrounding area [12]. Examples for multi-hop communication topologies are mesh networks (see Figure 2.2(b)) or hierarchical networks (see Figure 2.2(c)) [11]. The latter, as the name implies, possess a hierarchical structure in which not all sensor nodes have the same task assignments as the result of a clustering process. Clustered WSNs are covered in more detail in the next section.

\subsubsection{Sensor Node Clustering}

Large sensor networks, consisting of hundreds or even thousands of sensor nodes require scalable architectural and management strategies. In order to achieve the network scalability, sensor nodes can be grouped into clusters. Every cluster has a leader, called the cluster head, that may be just one of the sensor nodes or a sensor node provided with richer resources to fulfill additional tasks imposed by its function. Cluster heads can either be defined by the sensor nodes after deployment or preassigned before.

Diverse clustering algorithms have been proposed in the literature for wired networks and ad-hoc networks respectively. These algorithms mostly establish stable clusters and ensure node reachability and stability of the routes to the fusion center but cannot be directly applied to WSNs with their unique deployment and operational characteristics, since critical design goals of WSNs, e.g., long network lifetime, are completely ignored. Clustering algorithms specifically designed for WSNs have been proposed for a variety of applications. For an overview and a comparison of algorithms and detailed descriptions see $[16,17]$ and the references therein.

Algorithms can be classified based on the network architectural and operational model as well as the clustering objectives which are set in order to meet the applications' requirements and are therefore as diverse as the applications of WSNs themselves. The network architecture and the network operation depend on the type of sensor nodes used and their deployment. In a heterogeneous WSN some sensor nodes are empowered with distinct capabilities to act as cluster heads and are therefore pre-determined for that role. In a homogeneous WSN, though, all sensor nodes have equal computation, communication, and power capacities, can therefore be deployed randomly, and clustering and selection of cluster heads can be performed in a self-organizing manner. Depending on the application and the nature of the phenomenon to sense or monitor, the WSN can either work intermittently or continuously. Continuous monitoring requires periodic reporting and generates significant traffic so that in the long term, rotation of the cluster heads is required in order to evenly load all sensors. If the sensor nodes in the network are equipped with sufficient calculation power it is possible to perform data aggregation and data fusion by combining data and removing redundancy from data of several sensors. By that, the amount of transmitted data is reduced and hence substantial energy savings are obtained.

For many WSN applications the objectives of the clustering process are the same but their priority can vary significantly. Important objectives are the balancing of communication and computation load among the sensor nodes, optimization of network lifetime, robustness against the influences of the sometimes harsh environments where the sensor nodes are deployed, and connectivity requirements. Other objectives may arise for more special applications. Clustering should be performed such that computation and communication tasks of the 
WSN are distributed evenly over all the sensors. Distribution of the load among the sensor nodes is required to avoid overcharging the limited capacities of single sensor nodes and to prevent premature exhaustion of their energy supply and hence their dysfunction. It is therefore an important means to extend network lifetime. Adaptive clustering, i.e., algorithms that adapt the clustering of the network over time to parameter changes, additionally help to extend network lifetime. Moreover, robustness and fault-tolerance are enhanced. In harsh environments, sensor nodes are exposed to increased risk of malfunction and physical damage which could leave the WSN inoperable. In case of a sensor node malfunction that would harm the operability of the complete network or parts of it, re-clustering of the network is possible. Since this will usually interrupt the operation of the WSN one can define backup cluster heads that take over the function and recover the network from a failure. An obvious connectivity requirement for the clustering algorithm is that all sensor nodes have to be connected to the fusion center, but more sophisticated requirements could be imposed by limiting path lengths or delays.

For successful reconstruction, the field reconstruction scheme presented in this dissertation requires a clustering algorithm that yields clusters that establish stable sets of sampling. Further details about the requirements are presented in Chapter 3.

\subsubsection{Sensor Node Localization}

For many applications, such as the field reconstruction scheme we will present later, the sensor measurement data are useless without the location of the corresponding sensor node. Hence, (self-)localization capability is a highly desirable characteristic for nodes in WSNs. Sensor networks vary significantly from traditional cellular networks and Wireless Local Area Networks (WLANs) in that sensor nodes are assumed to be small, inexpensive, cooperative and deployed in large quantities. These features of sensor networks present unique challenges and opportunities for WSN localization.

Extensive research has been conducted on localization in wireless networks (see [14,18-21] and the references therein) and systems can be classified according to their properties, which we will summarize briefly in the following.

\section{Localization System Properties}

A localization system can provide either physical or symbolic information. A physical position is, for instance, expressed in a specific coordinate system, such as latitude and longitude using the WGS84 standard coordinate frame for the Earth. In contrast, a symbolic location encompasses abstract ideas of where something is located, e.g., in a city or next to a certain building. Depending on the resolution of a system, a physical position can usually be augmented to provide corresponding symbolic location information. In the simplest case this can be established by putting the physical positions of two objects in relation to derive, e.g., the symbolic location that one is above, beneath or next to the other. An absolute location system uses a common reference grid or coordinate system for all objects to be located. In a relative location system, however, each object can have its own reference point. The discrimination between absolute and relative positions refers primarily to the amount of information available at the different parts of the network. Knowing the absolute positions of the reference points, relative positions of all objects can be derived. Depending on the application, different 
parts of the network need to know about the position of the objects in the network. In some cases, it may be sufficient that the object's position is only known to a cluster head or the fusion center, in other cases it might be fundamental, e.g., due to privacy reasons, that only the objects themselves can determine their position using the infrastructure provided by the network. The accuracy of a localization system describes the resolution of the position information provided by the system. The corresponding precision as a measure of reliability denotes how often the system can be expected to achieve that accuracy. The scale of a localization system is the size of the area that is covered by the system and the number of objects it can locate. The system costs for installation, administration, maintenance, sensor nodes, and infrastructure strongly depend on the scale of the system. Depending on the infrastructure or the sensor nodes used, localization systems possess inherent limitations and do not work under all circumstances. For example, Global Navigation Satellite Systems (GNSSs) do not work indoors, in tunnels or under bridges. All these properties show that a localization system has to be designed attentively with the applications in mind.

\section{Localization Techniques}

We will give a brief overview of the techniques used to determine the position of sensor nodes in WSNs. Extensive surveys, comparisons, and detailed information about specific algorithms can be found in [18,22-24] and the references therein.

Sensor network localization algorithms are based on the knowledge of the absolute position of a few sensor nodes, called anchor nodes (or seed nodes). Their position is either known since they are deployed at points with known coordinates, or it can be obtained by external systems such as a GNSS. All the other sensor nodes, called non-anchor nodes, determine their position relative to the anchors. Non-anchor nodes can use range-based measurement techniques if they are direct neighbors to a sufficient number of anchor nodes. Then, estimates of the distance or the angles to their direct neighbors are used to calculate their position. Estimation of distances and angles is accomplished by triangulation, i.e., by measuring angles (angulation) and side lengths (lateration) of the triangles built by anchor nodes and non-anchor nodes and calculating the unknown lengths in these triangles based on plane trigonometry. Angles of Arrival (AOAs) can be measured using the amplitude or phase response of the sensor node's antenna or by so-called subspace techniques, e.g., MUSIC (MUltiple SIgnal Classification), ESPRIT (Estimation of Signal Parameters by Rotational Invariance Techniques) and their variations [18]. Distances between nodes can be measured indirectly from one-way or round-trip propagation times of sent messages, based on the Time Of Arrival (TOA) or the Time Difference Of Arrival (TDOA). These techniques are used in the IEEE 802.15.4a standard based on UWB $[25,26]$ to provide high precision localization capabilities with centimeter accuracy, but require accurate clock synchronization between the nodes in order to achieve high resolution positioning [27]. Distances between nodes can moreover be measured based on the Received Signal Strength (RSS) and propagation properties. Hybrid measurements, i.e., a combination of two or more of these measurements, are also possible in order to improve positioning accuracy [21,28].

The above mentioned localization techniques are based on a strong Line-Of-Sight (LOS) between nodes and anchor nodes. In case the LOS is obstructed or the measurements are contaminated by reflected or diffracted signals, Non-Line-Of-Sight (NLOS) error mitigation has to be performed [22]. If a LOS cannot be ensured and NLOS error mitigation is too complex, range-free localization techniques can be used which rely on connec- 
tivity information of the non-anchor nodes, i.e., which sensor node is connected to which other sensor nodes. A sensor node being in the transmission range of another sensor node defines a proximity constraint which can be exploited for localization.

\subsection{Estimation Theory}

The field reconstruction scheme that we will present in Chapter 3 is based on the estimation of channel coefficients from measurements of sensor nodes in a sensor network. We will therefore review a few basic concepts of estimation theory [29].

Estimation theory deals with estimating parameters $\boldsymbol{\theta}=\left(\theta_{1} \ldots \theta_{N}\right)^{T}$ from measured data $\mathbf{x}=\left(x_{1} \ldots x_{M}\right)^{T}$. The parameter vector $\boldsymbol{\theta}$ cannot be observed directly or not measured accurately, so that it has to be inferred from the measured data vector $\mathrm{x}$ that depends on the parameters. The dependence is established, for instance, by a physical phenomenon and must be known to some extent in order to be able to estimate $\boldsymbol{\theta}$ from $\mathbf{x}$. The dependence can be either deterministic or stochastic. For a deterministic dependence the observed data are modeled by a known function $s(\cdot)$ impaired by unknown deterministic errors $\mathbf{w}$, i.e., $\mathbf{x}=s(\boldsymbol{\theta})+\mathbf{w}$. Stochastic dependence is described by the Probability Density Function (pdf) of $\mathbf{x}$, either parameterized by $\boldsymbol{\theta}$ (if the parameter is deterministic), i.e., $f(\mathbf{x} ; \boldsymbol{\theta})$, or conditioned on $\boldsymbol{\theta}$ (if it is stochastic), i.e., $f(\mathbf{x} \mid \boldsymbol{\theta})$. The parameter vector $\boldsymbol{\theta}$ is either modeled as deterministic vector (classical estimation) or as a random vector (Bayesian estimation). The estimator function $\hat{\boldsymbol{\theta}}(\mathbf{x})$ calculates an estimate $\hat{\boldsymbol{\theta}}$ for the parameter vector $\boldsymbol{\theta}$ only from the measured data $\mathrm{x}$. The design of the estimator function is based on the dependence between $\theta$ and $\mathbf{x}$, i.e., on $s(\cdot), f(\mathbf{x} ; \boldsymbol{\theta})$ or $f(\mathbf{x} \mid \boldsymbol{\theta})$ and $f(\boldsymbol{\theta})$ respectively. The goal of parameter estimation is to attain an estimate $\hat{\boldsymbol{\theta}}$ that is close to the true value $\boldsymbol{\theta}$, i.e., the estimation error $\mathbf{e}=\hat{\boldsymbol{\theta}}-\boldsymbol{\theta}$ or its square should be small in a certain sense.

The estimation problem can be approached from two different perspectives, the classical estimation on the one side and the Bayesian estimation on the other side. We will give a short overview of both approaches in the following.

\subsubsection{Classical Estimation}

In classical estimation the parameter $\boldsymbol{\theta}$ of interest is assumed to be deterministic but unknown. The assumption of $\boldsymbol{\theta}$ being deterministic follows from the fact that no prior statistical information about the parameters is available. The dependence of the random measured data $\mathbf{x}$ is described by a family of pdfs $f(\mathbf{x} ; \boldsymbol{\theta})$ parameterized by $\boldsymbol{\theta}$. The interest typically lies in unbiased estimators, i.e., estimators for which $\operatorname{bias}\{\hat{\boldsymbol{\theta}}\}=\mathrm{E}\{\mathbf{e}\}=\mathrm{E}\{\hat{\boldsymbol{\theta}}(\mathbf{x})\}-\boldsymbol{\theta}=\mathbf{0}$. The Mean Square Error (MSE) of the estimate $\hat{\boldsymbol{\theta}}$ equals

$$
\varepsilon_{\hat{\boldsymbol{\theta}}}(\boldsymbol{\theta})=\frac{1}{M} \mathrm{E}\left\{\|\mathbf{e}\|^{2}\right\}=\frac{1}{M} \mathrm{E}\left\{\|\hat{\boldsymbol{\theta}}(\mathbf{x})-\boldsymbol{\theta}\|^{2}\right\}=\operatorname{var}\{\hat{\boldsymbol{\theta}}\}+\frac{1}{M}\|\operatorname{bias}\{\hat{\boldsymbol{\theta}}\}\|^{2},
$$

which for an unbiased estimator equals $\operatorname{var}\{\hat{\boldsymbol{\theta}}\}=\frac{1}{M} \mathrm{E}\left\{\|\hat{\boldsymbol{\theta}}(\mathbf{x})-\mathrm{E}\{\hat{\boldsymbol{\theta}}\}\|^{2}\right\}$, i.e., the MSE equals the variance. Among all unbiased estimators, the estimator with the minimum variance (and therefore the minimum MSE) for all $\boldsymbol{\theta}$ is called the Minimum Variance Unbiased (MVU) estimator. 
An unbiased classical estimator $\hat{\boldsymbol{\theta}}(\mathbf{x})$ is said to be efficient if its covariance matrix attains the Cramér-Rao Lower Bound (CRLB), i.e., if

$$
\operatorname{cov}_{\hat{\boldsymbol{\theta}}}(\boldsymbol{\theta})=\mathbf{J}^{-1}(\boldsymbol{\theta})
$$

Here, $\mathbf{J}(\boldsymbol{\theta})$ is the Fisher information matrix (see [29] for definition and properties). If an efficient estimator exists, it is the MVU estimator and the Maximum Likelihood (ML) estimator simultaneously. If an efficient estimator does not exist, it is however still possible that an MVU estimator exists. Unfortunately, the existence of the MVU estimator is not guaranteed or it often cannot be found. Therefore suboptimal but more practical estimators, like the ML estimator or the Best Linear Unbiased Estimator (BLUE), are used.

The ML estimator chooses that $\boldsymbol{\theta}$ that maximizes the likelihood function for the observed $\mathrm{x}$, i.e.,

$$
\hat{\boldsymbol{\theta}}_{\mathrm{ML}}(\mathbf{x}) \triangleq \underset{\boldsymbol{\theta}}{\arg \max } f(\mathbf{x} ; \boldsymbol{\theta}) .
$$

Sometimes, this maximization problem cannot be solved in closed form but only by numerical techniques. This may be computationally too complex and it is therefore often reasonable to constrain the estimator to be a linear function of the data $\mathbf{x}$. The BLUE minimizes the variance under the constraint of unbiasedness and linearity. Assuming a linear data model $\mathbf{x}=\mathbf{H} \boldsymbol{\theta}+\mathbf{w}$, the BLUE is given by

$$
\hat{\boldsymbol{\theta}}_{\mathrm{BLUE}}(\mathbf{x})=\left(\mathbf{H}^{T} \mathbf{C}_{\mathbf{w}}^{-1} \mathbf{H}\right)^{-1} \mathbf{H}^{T} \mathbf{C}_{\mathbf{w}}^{-1}\left(\mathbf{x}-\boldsymbol{\mu}_{\mathbf{w}}\right),
$$

with $\mathbf{C}_{\mathbf{w}}$ being the covariance matrix and $\boldsymbol{\mu}_{\mathrm{w}}$ the mean of the random vector $\mathbf{w}$.

Without any statistical model for the parameter $\boldsymbol{\theta}$ or the data $\mathbf{x}$, one assumes a deterministic model $s(\cdot)$ for the dependence of the data on the parameter. The data model is deterministic up to a modeling or measurement error w, i.e., $\mathbf{x}=s(\boldsymbol{\theta})+\mathbf{w}$. The Least Squares (LS) estimator selects the $\boldsymbol{\theta}$ for which $s(\boldsymbol{\theta})$ is closest to the data $\mathrm{x}$ in a least squares sense,

$$
\hat{\boldsymbol{\theta}}_{\mathrm{LS}}(\mathbf{x}) \triangleq \underset{\boldsymbol{\theta}}{\arg \min }\|\mathbf{x}-s(\boldsymbol{\theta})\|^{2}=\left(\mathbf{H}^{T} \mathbf{H}\right)^{-1} \mathbf{H}^{T} \mathbf{x}=\mathbf{H}^{\#} \mathbf{x}
$$

Here, the matrix $\mathbf{H}^{\#}=\left(\mathbf{H}^{T} \mathbf{H}\right)^{-1} \mathbf{H}^{T}$ is the Moore-Penrose pseudo-inverse of $\mathbf{H}$. In order to emphasize the contributions of those data that are considered more reliable, weighting factors can be introduced in the error criterion via a symmetric, positive definite weighting matrix $\mathbf{W}$. We then get the Weighted Least Squares (WLS) estimator

$$
\hat{\boldsymbol{\theta}}_{\mathrm{WLS}}(\mathbf{x}) \triangleq \underset{\boldsymbol{\theta}}{\arg \min }\|\mathbf{x}-s(\boldsymbol{\theta})\|_{\mathbf{W}}^{2} \triangleq \underset{\boldsymbol{\theta}}{\arg \min }(\mathbf{x}-\mathbf{H} \boldsymbol{\theta})^{T} \mathbf{W}(\mathbf{x}-\mathbf{H} \boldsymbol{\theta})=\left(\mathbf{H}^{T} \mathbf{W H}\right)^{-1} \mathbf{H}^{T} \mathbf{W} \mathbf{x} .
$$

If statistical prior knowledge of the measurement error $\mathbf{w}$ (mean $\boldsymbol{\mu}_{\mathrm{w}}$ and covariance matrix $\mathbf{C}_{\mathbf{w}}$ ) is available, the WLS estimator with data $\mathbf{x}^{\prime}=\mathbf{x}-\boldsymbol{\mu}_{\mathbf{w}}$ and weighting matrix $\mathbf{W}=\mathbf{C}_{\mathbf{w}}^{-1}$ coincides with the BLUE. For a linear-Gaussian model, the BLUE (as well as the WLS estimator) is the MVU and also equals the ML estimator.

\subsubsection{Bayesian Estimation}

In Bayesian estimation both the measured data $\mathrm{x}$ and the parameter $\boldsymbol{\theta}$ are modeled as random. We use the statistical information of $\mathbf{x}$ and $\boldsymbol{\theta}$, i.e., the pdf $f(\boldsymbol{\theta})$ and the conditional pdf $f(\mathbf{x} \mid \boldsymbol{\theta})$ that characterizes the 
dependence of $\mathrm{x}$ on $\boldsymbol{\theta}$. Bayesian estimation aims at minimizing a suitable average measure of the estimation error $\mathbf{e}=\hat{\boldsymbol{\theta}}(\mathbf{x})-\boldsymbol{\theta}$. The general Bayesian estimator is given by

$$
\hat{\boldsymbol{\theta}}_{\mathrm{B}}(\mathbf{x}) \triangleq \underset{\hat{\boldsymbol{\theta}}}{\arg \min } \mathrm{E}\{C(\hat{\boldsymbol{\theta}}-\boldsymbol{\theta})\}=\underset{\hat{\boldsymbol{\theta}}}{\arg \min } \int_{\boldsymbol{\theta}} C(\hat{\boldsymbol{\theta}}-\boldsymbol{\theta}) f(\mathbf{x} \mid \boldsymbol{\theta}) f(\boldsymbol{\theta}) d \boldsymbol{\theta},
$$

with a scalar-valued cost function $C(\cdot)$. Choosing the cost function to be quadratic, we obtain the Minimum Mean Square Error (MMSE) estimator

$$
\hat{\boldsymbol{\theta}}_{\mathrm{MMSE}}(\mathbf{x}) \triangleq \underset{\hat{\boldsymbol{\theta}}}{\arg \min } \mathrm{E}\left\{\|\hat{\boldsymbol{\theta}}-\boldsymbol{\theta}\|^{2}\right\}=\int_{\boldsymbol{\theta}} \boldsymbol{\theta} f(\boldsymbol{\theta} \mid \mathbf{x}) d \boldsymbol{\theta}=\mathrm{E}\{\boldsymbol{\theta} \mid \mathbf{x}\} .
$$

In general, this is a nonlinear function of the measurement data $\mathbf{x}$ and often it cannot be determined in closed form or its implementation is computationally costly. It is therefore sometimes reasonable to constrain the estimator to be a linear function of the data. The resulting Linear Minimum Mean Square Error (LMMSE) estimator

$$
\hat{\boldsymbol{\theta}}_{\mathrm{LMMSE}}(\mathbf{x})=\boldsymbol{\mu}_{\boldsymbol{\theta}}+\mathbf{C}_{\mathbf{x}, \boldsymbol{\theta}}^{T} \mathbf{C}_{\mathbf{x}}^{-1}\left(\mathbf{x}-\boldsymbol{\mu}_{\boldsymbol{\theta}}\right),
$$

with the covariance matrix $\mathbf{C}_{\mathbf{x}}$ of $\mathbf{x}$ and the cross-covariance matrix $\mathbf{C}_{\mathbf{x}, \boldsymbol{\theta}}=\mathrm{E}\left\{\mathbf{x} \boldsymbol{\theta}^{T}\right\}$ of $\mathbf{x}$ and $\boldsymbol{\theta}$, is suboptimal in general, but can always be calculated in closed form. For jointly Gaussian $\mathrm{x}$ and $\boldsymbol{\theta}$, the LMMSE estimator is equal to the MMSE estimator.

Using a Bayesian estimator with the cost function

$$
C(\mathbf{e})= \begin{cases}1, & \|e\|>\delta \\ 0, & \|e\| \leq \delta\end{cases}
$$

in the limit for threshold $\delta \rightarrow 0$ gives the Maximum A Posteriori (MAP) estimator

$$
\hat{\boldsymbol{\theta}}_{\mathrm{MAP}}(\mathbf{x})=\underset{\theta}{\arg \max } f\left(\boldsymbol{\theta}_{m} \mid \mathbf{x}\right),
$$

that maximizes the posterior pdf of the vector $\boldsymbol{\theta}$.

An unbiased Bayesian estimator $\hat{\boldsymbol{\theta}}(\mathbf{x})$ is said to be efficient if its error covariance matrix attains the CRLB, i.e., if

$$
\operatorname{cov}\{\mathbf{e}\}=\mathbf{L}^{-1}
$$

Here, $\mathbf{L}$ is the Bayesian information matrix (see [30] for the definition and properties). If an efficient estimator exists, it is the MAP estimator and the MMSE estimator simultaneously. An efficient Bayesian estimator exists if and only if the posterior pdf $f(\boldsymbol{\theta} \mid \mathbf{x})$ is Gaussian.

\subsection{Convex Optimization}

In this section we will introduce a few basic concepts used in mathematical optimization in general and in convex optimization in particular. Convex optimization is widely used in application areas such as control, circuit design, economics and finance, networking, and statistics and machine learning. We will only give 
a short introduction in order to be able to understand the basic terms used in Chapter 4. For an extensive introduction to optimization problems with a strong focus on convex optimization see [10,31].

In general, a mathematical optimization problem, or just optimization problem, has the form [10]

$$
\begin{array}{ll}
\operatorname{minimize} & f_{0}(\mathbf{x}) \\
\text { subject to } & f_{i}(\mathbf{x}) \leq 0, \quad i=1, \ldots, m \\
& h_{i}(\mathbf{x})=0, \quad i=1, \ldots, p
\end{array}
$$

The equivalent dual maximization problem can be solved by minimizing the negative objective function. Here, the vector $\mathbf{x}=\left(x_{1} \ldots x_{n}\right)^{T}$ is the optimization variable of the problem, the function $f_{0}: \mathbb{R}^{n} \rightarrow \mathbb{R}$ is the objective function or cost function, the functions $f_{i}: \mathbb{R}^{n} \rightarrow \mathbb{R}, i=1, \ldots, m$, are the inequality constraint functions, and the functions $h_{i}: \mathbb{R}^{n} \rightarrow \mathbb{R}, i=1, \ldots, p$, are the equality constraint functions. If there are no constraints, the problem is called unconstrained. The domain of the problem is the set of points for which the objective function and all constraint functions are defined, i.e.,

$$
\mathcal{D}=\bigcap_{i=0}^{m} \operatorname{dom} f_{i} \cap \bigcap_{i=1}^{p} \operatorname{dom} h_{i} .
$$

A point $\mathbf{x} \in \mathcal{D}$ is feasible if it satisfies the inequality and equality constraints. The set of all feasible points is called the feasible set. The solution of the optimization problem (2.1) is the optimal vector $\mathrm{x}^{\star}$ that attains the smallest objective value among all vectors in the feasible set. Hence, for any $\mathbf{z} \in \mathcal{D}$ with $f_{1}(\mathbf{z}) \leq 0, \ldots, f_{m}(z) \leq 0$, we have $f_{0}(\mathbf{z}) \geq f_{0}\left(\mathbf{x}^{\star}\right)$.

Convex optimization problems are a class of mathematical optimization problems, characterized by particular forms of the objective and constraint functions. Specifically, for a convex optimization problem, the objective function and the inequality constraints must be convex and the equality constraint functions must be affine. For a mathematical optimization problem, the property of convexity is of high importance because it allows to formulate first order optimality criteria that are sufficient for a global optimum.

The feasible set of a convex optimization problem is a convex set, which is a set $\mathcal{C}$ that contains every point on the line segment connecting any two points in $\mathcal{C}$, i.e., for any $\mathbf{x}, \mathbf{y} \in \mathcal{C}$ and any $\theta$ with $0 \leq \theta \leq 1$, we have

$$
\theta \mathbf{x}+(1-\theta) \mathbf{y} \in \mathcal{C}
$$

A function $f_{i}: \mathbb{R}^{n} \rightarrow \mathbb{R}$ is a convex function if its domain $\operatorname{dom} f$ is a convex set and if for all $\mathbf{x}, \mathbf{y} \in \operatorname{dom} f$, and $\theta$ with $0 \leq \theta \leq 1$, we have

$$
f(\theta \mathbf{x}+(1-\theta) \mathbf{y}) \leq \theta f(\mathbf{x})+(1-\theta) f(\mathbf{y})
$$

A fundamental property of convex optimization problems is that if any locally optimal point exists, then it is also globally optimal. This fact simplifies the optimization and allows for very effective solution methods. Unlike many general optimization problems, most convex optimization problems can be efficiently solved, both in theory and practice. Usually the difficulty lies in recognizing and formulating convex optimization problems after which solving them often is straight forward in principle. 


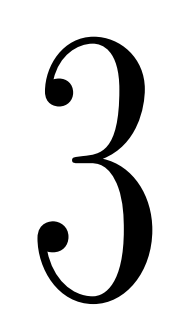

\section{Distributed Field Reconstruction}

We use the theory and algorithms developed for so-called shift-invariant spaces to develop a novel distributed architecture for sampling and reconstruction of time-varying non-bandlimited physical fields in Wireless Sensor Networks (WSNs). We introduce hybrid shift-invariant spaces, that generalize conventional shift-invariant spaces and can adapt to local smoothness properties of the field. Using shift-invariant spaces with compactly supported generator functions allows us to split the global reconstruction into several smaller local problems that can be solved independently. Capitalizing on the sparsity of the matrices involved in the reconstruction, we propose direct and iterative reconstruction algorithms whose complexity per time slot scales only linearly with the number of sensor nodes. We furthermore analyze the impact of sensor node localization errors on the mean square error of the reconstructed field. Numerical simulations illustrate that the proposed field reconstruction scheme performs as good as bandlimited reconstruction and is less sensitive to sensor node location errors while providing a significant reduction in computational complexity. 


\subsection{Background and State of the Art}

The problem of sampling and reconstruction of physical fields has been previously addressed in the literature under the often unrealistic assumption that the field is spatially strictly bandlimited. An efficient scheme for the reconstruction of bandlimited fields using a trade-off between spatial oversampling and sensor quantizer resolution was presented in [32]. The accuracy of linear bandlimited reconstruction in WSN with non-uniform sampling was studied in [33] using random matrix theory. In temporal sampling applications, sufficient bandlimitation can be ensured by preceding the sampling with continuous-time anti-aliasing filters. This is in stark contrast to WSN where spatial anti-aliasing filtering is inherently impossible since the continuous-space field cannot be accessed or pre-processed. At the same time the assumption of a bandlimited field is too restrictive for many physical scenarios. In fact, non-wave fields (e.g., electrostatic fields, gravitation fields, diffusion fields in liquids and gases) cannot be assumed to be strictly bandlimited (see e.g. [34]). In [35] it was shown that significant oversampling is required to achieve small reconstruction errors when using bandlimited reconstruction for non-bandlimited fields.

In this chapter we introduce new schemes for reconstructing non-bandlimited spatio-temporal fields in WSN. We propose to use shift-invariant spaces [8] instead of bandlimited spaces as models for the sensing of smooth spatio-temporal fields and we further introduce an extension termed hybrid shift-invariant spaces that allows the amount of smoothness of the field to be adapted locally. We extend and adapt the One-Dimensional (1-D) reconstruction algorithm from [9] to the Two-Dimensional (2-D) case. Our approach is highly local in the sense that reconstructing the field at a certain position requires only samples from a small spatial neighborhood. A theoretical analysis provides insights regarding the sensor node (i.e., sampling) density required for perfect reconstruction to be feasible. We develop a clustered WSN architecture that is matched to the localized nature of our field models. Within each cluster, a cluster head collects measurements from all sensor nodes within the cluster and performs local field reconstruction. This architecture allows for a graceful transition between fully centralized field reconstruction (one cluster and a fusion center) and completely decentralized operation (each sensor node is a cluster head and performs the same tasks). Depending on the application context, the communication and computational burden can thus be distributed among the sensor nodes in a flexible manner. We discuss various implementations of the actual field reconstruction algorithm using either direct or iterative solvers for the underlying sparse linear system of equations. It turns out that in all cases the computational complexity scales only linearly with the number of sensor nodes. Iterative solvers are particularly attractive for tracking time-varying spatial fields since here the number of iterations can be significantly reduced by using the solution obtained in the previous time-step as initialization. We further show that ill-conditioned systems of equations result from regions with too low sensor node density. Excluding these regions from the reconstruction improves the reconstruction quality in the rest of the field. We study how quantization and measurement noise affect the stability and the quality of the field reconstruction. Furthermore, we provide semi-analytical and numerical investigations of the impact of sensor node localization errors on the performance of our field reconstruction method. It turns out that shift-invariant interpolation is much more robust to localization errors than bandlimited interpolation. Finally, we provide extensive numerical simulations to illustrate the excellent reconstruction performance of the proposed scheme under a variety of operating conditions. 
Our scheme is highly attractive since it is flexible, features very low computational complexity, requires low communication overhead, is robust against quantization and localization errors, and requires only the sensor node positions to be known. We note that due to the favorable computational and communication requirements of our method, consensus- or diffusion-based implementations (e.g., [36-39]) that distribute the computational complexity across sensor nodes at the expense of increased communication overhead are less interesting in our context.

\subsection{System Model}

\subsubsection{Wireless Sensor Network Architecture}

We consider a WSN consisting of $I$ sensor nodes, which are deployed over a given region $\mathcal{A}$ to monitor a 2-D spatio-temporal physical field $h(x, y ; t)$. Here, $x$ and $y$ denote the spatial coordinates and $t$ denotes time. The position of sensor node $i$ is given by $\left(x_{i}, y_{i}\right)$ and its measurement during the $n$th sampling period is denoted by $h_{i}(n)$. In this chapter we will mostly be concerned with spatial interpolation/estimation of the field $h(x, y ; t)$ for fixed time $t$; temporal interpolation can be performed using a variety of methods for smooth temporal models [40]. We consider a clustered system architecture (see Figure 3.1) where the sensor nodes are grouped into $M$ clusters $\mathcal{C}_{m}$ of cardinality $I_{m}, m=1, \ldots, M$, that monitor the subregions $\mathcal{A}_{m}\left(\bigcup_{m=1}^{M} \mathcal{A}_{m}=\mathcal{A}\right)$. These subregions may overlap and may have different areas. For simplicity of exposition, however, we assume disjoint subregions $\mathcal{A}_{m}$ in the following. In case the $\mathcal{A}_{m}$ are not disjoint, our method can be augmented by averaging the local field reconstruction results in the overlapping regions (see Section 3.4.3 for more details).

In each cluster $\mathcal{C}_{m}$ there is one node, termed the cluster head, which reconstructs the field $h(x, y ; n T)$ for $(x, y) \in \mathcal{A}_{m}$ according to the algorithm outlined in Section 3.4. To this end, it collects the measurements $h_{i}(n)$ and the corresponding positions $\left(x_{i}, y_{i}\right)$ of all sensor nodes within the cluster. This requires that each sensor node has determined its own position (see Section 2.1.5 for more details about sensor node localization). We note that as an alternative, the cluster heads can forward intermediate results to a fusion center, which then globally reconstructs the field.

The choice of the WSN clusters and cluster heads has a crucial impact on the performance of the sensor network. Since the cluster heads performs all the computations necessary for the reconstruction and may be required to transmit their results to a global fusion center, they consume more energy than the other sensor nodes who only transmit the measured data to the cluster head. This imbalance in energy consumption can be overcome by letting all sensor nodes act as cluster heads in a round robin fashion, thereby distributing the computation and communication load uniformly over all nodes in a cluster. Besides improved energy efficiency, WSN clustering offers additional advantages like reduced channel contention and fewer packet collisions. For our field reconstruction scheme it is desirable to choose the clusters such that they form stable sampling sets [8] and increase the network lifetime by reducing the communication overhead. More information about grouping WSN nodes into clusters and identifying cluster heads can be found in Section 2.1.4.

We note that local field reconstruction within a cluster, i.e., without requiring data from the other clusters, is possible due to the localized nature of the proposed non-bandlimited field model (see Section 3.3.1 for details). 


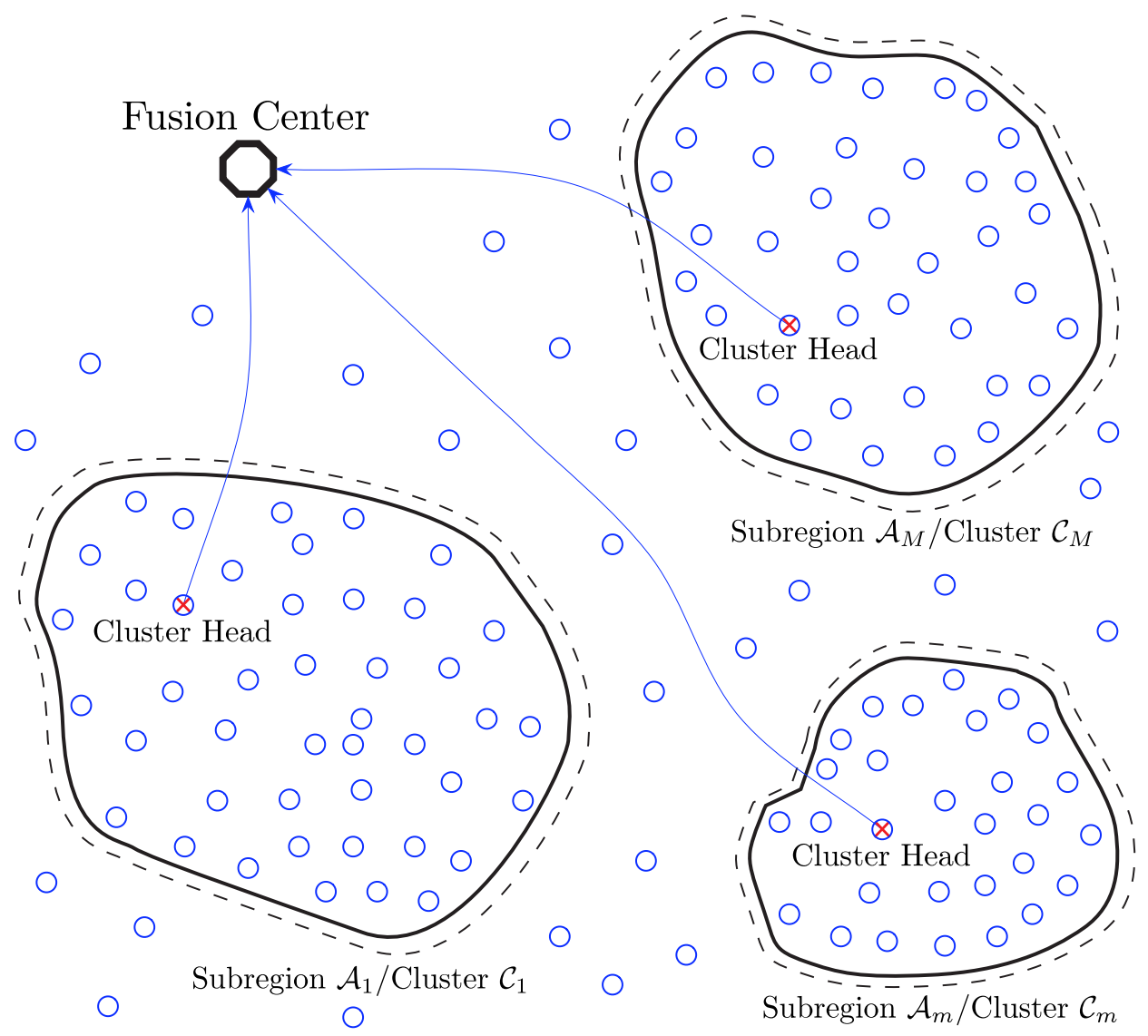

Figure 3.1: Illustration of clustered WSN architecture for field reconstruction (not all clusters shown). The field is reconstructed locally within every subregion (solid lines) by the respective sensor node cluster. If desired, the result is forwarded to a fusion center. The local field reconstruction involves samples from the slightly larger subregions (circumscribed by dashed lines).

The advantages of our clustered WSN architecture are its scalable distributed nature that entails reduced communication and computational complexity, and improved ability to locally adapt the field model (see Section 3.3.3). In addition, poor field reconstruction quality within the region $\mathcal{A}_{m}$ corresponding to cluster $\mathcal{C}_{m}$ is a local effect that does not degrade the reconstruction quality within $\mathcal{A} \backslash \mathcal{A}_{m}$.

\subsubsection{Measurement Model}

In practice, the sensor node measurements are impaired by measurement and quantization noise. The measurements taken by the sensor nodes are quantized noisy samples of the physical field $h(x, y ; t)$. The quantized measurements are therefore given by $h_{i}(n)=Q\left\{h\left(x_{i}, y_{i} ; n T\right)+w_{i}(n T)\right\}$, where $Q\{\cdot\}$ represents the action of the quantizer, $T$ is the temporal sampling period, and $w_{i}(n T)$ denotes measurement noise. For simplicity, we assume that each sensor node uses a uniform quantizer with a resolution of $B$ bits and dynamic range $\pm h_{\max }$. We assume that the dynamic range is large enough to avoid clipping, i.e., $h(x, y ; t) \in\left[-h_{\max }, h_{\max }\right]$. The maximum magnitude of the quantization error in this case is given by $2^{-B} h_{\max }$. The quantizer resolution $B$ not only determines the field reconstruction quality but also the communication requirements for transmitting 
the measurements to the cluster heads. Since typically many measurements will be taken over time and repeated transmission of a large number of bits can consume a significant fraction of a sensor node's battery power, the choice of $B$ can strongly influence network lifetime.

\subsection{Field Model - Shift-Invariant Spaces}

We first introduce conventional shift-invariant spaces (e.g., $[8,41])$ as mathematical models for the spatiotemporal field $h(x, y ; t)$. Our terminology is adapted to the WSN setup and our discussion is restricted to the 2-D case throughout the dissertation. This basic model will afterwards be extended to hybrid shift-invariant spaces in order to locally adapt the model to the field to be reconstructed.

\subsubsection{Conventional Shift-Invariant Spaces}

We model the smooth spatio-temporal field via a shift-invariant space, i.e., we assume $h(x, y ; t) \in V(g)$, where $V(g)$ is a linear subspace of $L^{2}\left(\mathbb{R}^{2}\right)$ comprising all functions that can be represented as weighted superposition of spatial translates of some generator function $g(x, y) \in L^{2}\left(\mathbb{R}^{2}\right)$, i.e.,

$$
V(g) \triangleq\left\{f \in L^{2}\left(\mathbb{R}^{2}\right): f(x, y)=\sum_{(k, l) \in \mathbb{Z}^{2}} c_{k, l} g\left(x-k D_{x}, y-l D_{y}\right)\right\},
$$

where $c_{k, l} \in l_{2}\left(\mathbb{Z}^{2}\right)$. Here,

$$
L^{2}\left(\mathbb{R}^{2}\right)=\left\{f(x, y): \iint_{\mathbb{R}^{2}}|f(x, y)|^{2} d x d y<\infty\right\}
$$

stands for the space of square-integrable 2-D functions and

$$
l_{2}\left(\mathbb{Z}^{2}\right)=\left\{c_{k, l}: \sum_{(k, l) \in \mathbb{Z}^{2}}\left|c_{k, l}\right|^{2}<\infty\right\}
$$

denotes the space of square-summable 2-D sequences. Without loss of generality, we will assume $D_{x}=D_{y}=$ 1 throughout the dissertation, which can always be ensured by appropriately rescaling the spatial coordinates $x$ and $y$. To guarantee the stability of the representation above, we further assume that the set of translates $\{g(x-k, y-l)\}_{(k, l) \in \mathbb{Z}^{2}}$ forms a Riesz basis for $V(g)[9,42]$.

Bandlimited spaces are a special case of shift-invariant spaces, obtained with the separable sinc-type generator function

$$
g_{\mathrm{BL}}(x, y) \triangleq \frac{\sin \left(B_{x} \pi x\right)}{B_{x} \pi x} \frac{\sin \left(B_{y} \pi y\right)}{B_{y} \pi y}
$$

where $B_{x}$ and $B_{y}$ denote the spatial bandwidths. Since $g_{\mathrm{BL}}(x, y)$ decays very slowly, the corresponding space $V\left(g_{\mathrm{BL}}\right)$ is highly non-local, i.e., the field value $h(x, y ; n T) \in V\left(g_{\mathrm{BL}}\right)$ depends on samples that are arbitrarily far away from $(x, y)$. This is clearly undesirable and makes the space of bandlimited functions often unsuitable for numerical implementation. Moreover, physical fields that are measured in applications tend to have components 
that decay for higher frequencies, but these functions are not bandlimited in the strict sense. This motivates the advantageous use of non-bandlimited models that retain some of the simplicity and structure of bandlimited models but are more amenable to numerical implementation and are more flexible in the approximation of real data. Shift-invariant spaces using generator functions with compact support, such as Basis-splines (B-splines), to be introduced in Section 3.3.2, are such models. They can be modified to be even more flexibly adaptable to the physical field, as we will show in Section 3.3.3. Sampling in non-bandlimited shift-invariant spaces is a suitable and realistic model for many applications, e.g., for taking into account real acquisition and reconstruction devices, for modeling signals with smoother spectrum than is the case with bandlimited functions, or for numerical implementation. The application requirements can often be met by choosing appropriate generator functions $g(x, y)$. This may mean that the functions $g(x, y)$ have a shape corresponding to a particular impulse response of a device, that they are compactly supported, or that they have a smoothly decaying Fourier transform [8].

\subsubsection{Compactly Supported Generator Functions}

The undesirable non-local nature of band-limited spaces motivates us to propose the use of shift-invariant spaces with a compactly supported generator function. The support of $g(x, y)$ is defined as

$$
\mathcal{S} \triangleq \operatorname{cl}\left\{(x, y) \in \mathbb{R}^{2}:|g(x, y)|>0\right\}
$$

where $\operatorname{cl}\{\cdot\}$ denotes topological closure. Hence the support is the closure of the set of points $(x, y)$ where the function $g(x, y)$ is non-zero. A particularly useful class of compactly supported generator functions is given by B-splines that are piecewise-polynomial functions having minimal support with respect to a given degree and are commonly used for interpolation purposes [43]. The advantage of polynomials is that they can be evaluated, differentiated, and integrated easily and in finitely many steps using only basic arithmetic operations [43,44]. Specifically, we will use 2-D spline functions in the following, constructed as

$$
b_{N}(x, y)=\tilde{b}_{N}(x) \tilde{b}_{N}(y)
$$

where the 1-D splines of order $N$ (see Figure 3.2) are defined via the $N$-fold convolution

$$
\tilde{b}_{N}(x) \triangleq \Pi(x) \underbrace{* \Pi(x) \ldots * \Pi(x)}_{N \text { times }} .
$$

Here, the rectangular function $\Pi(x)=\tilde{b}_{0}(x)$ is defined as

$$
\Pi(x) \triangleq \begin{cases}1, & -\frac{1}{2} \leq x<\frac{1}{2} \\ 0, & \text { else. }\end{cases}
$$

Alternatively, the B-splines can be accurately calculated using the Cox-de Boor recursion [44]. Both methods for the calculation of B-splines are discussed in more detail in Appendix 3.B. The support of the 2-D splines is given by $\mathcal{S}=[-(N+1) / 2,(N+1) / 2] \times[-(N+1) / 2,(N+1) / 2]$. In our application context, the most important implication of the compact support of $b_{N}(x, y)$ is the strongly localized nature of $V\left(b_{N}\right)$. According 

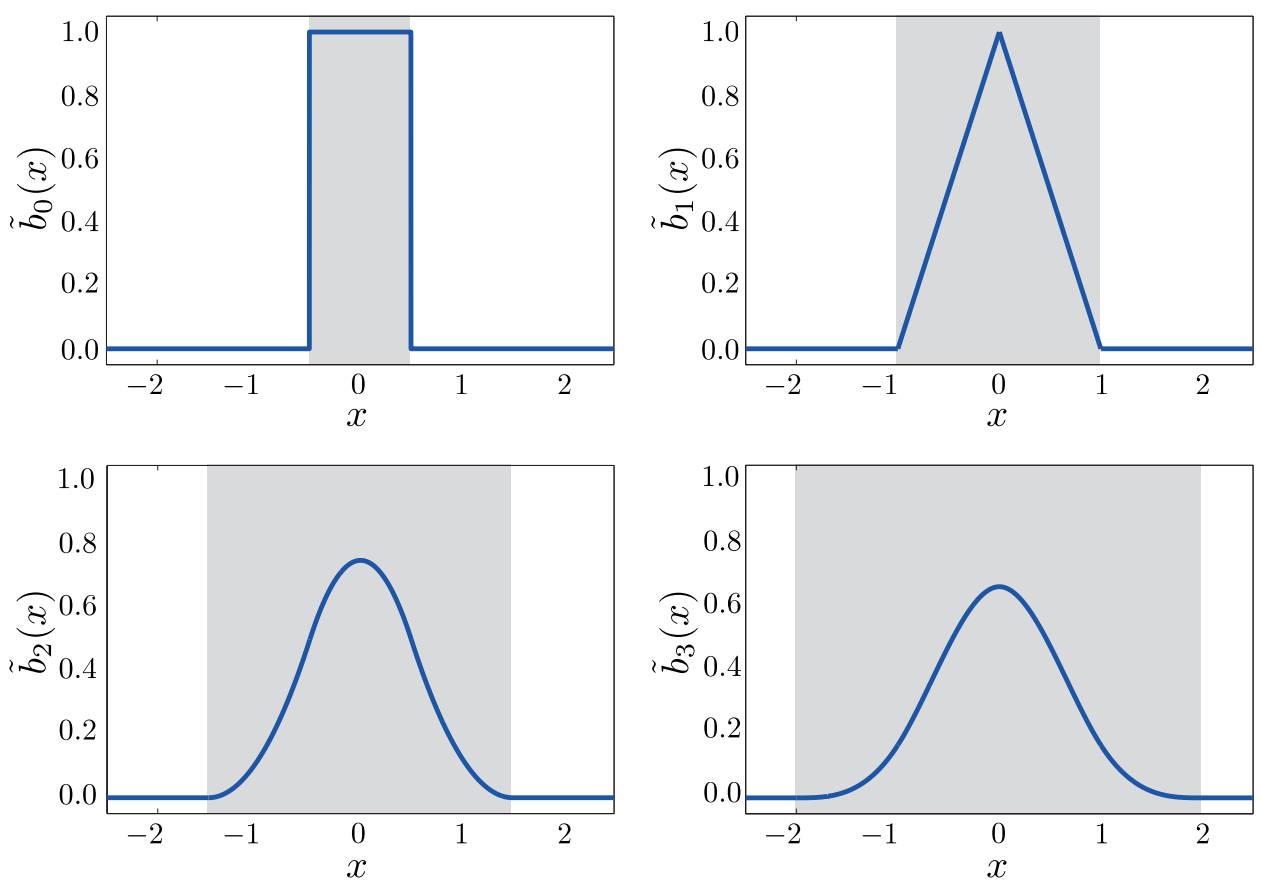

Figure 3.2: Illustration of 1-D B-splines of order $N \in\{0,1,2,3\}$. The support is shown in gray shade.

to the representation $f(x, y)=\sum_{k, l} c_{k, l} b_{N}(x-k, y-l)$, the field $f(x, y)$ at any position $(x, y)$ depends only on the $(N+1)^{2}$ coefficients $c_{k, l}$ for which $(x-k, y-l) \in \mathcal{S}$. Due to the compact support of $b_{N}(x, y)$, the shift-invariant spaces $V\left(b_{N}\right)$ are not strictly bandlimited. However, the elements of $V\left(b_{N}\right)$ are smooth, with the degree of smoothness increasing with growing $N$ (see Figure 3.2).

\subsubsection{Hybrid Shift-Invariant Spaces}

With the clustered WSN architecture described in Section 3.2, the region $\mathcal{A}$ is divided into $M$ subregions $\mathcal{A}_{m}$. This motivates us to model the spatio-temporal field using potentially different generator functions in every subregion. To this end, we introduce the hybrid shift-invariant spaces

$$
V(\mathcal{G}) \triangleq\left\{f \in L^{2}\left(\mathbb{R}^{2}\right): f(x, y)=\sum_{m=1}^{M} \sum_{(k, l) \in \mathbb{A}_{m}} c_{k, l} g_{m}(x-k, y-l)\right\},
$$

where $\mathcal{G}=\left\{g_{m}(x, y)\right\}_{m=1, \ldots, M}, \mathbb{A}_{m}=\mathbb{Z}^{2} \cap\left(\mathcal{A}_{m}+\mathcal{S}\right)$, and $c_{k, l} \in l^{2}\left(\mathbb{Z}^{2}\right)$. This defines a linear subspace of $L^{2}\left(\mathbb{R}^{2}\right)$ that comprises all fields that can be represented as weighted superposition of spatial translates of the generator functions $g_{m}(x, y)$. Using different generator functions $g_{m}(x, y)$ in the subregions $\mathcal{A}_{m}$ allows us to locally adapt the smoothness properties of the field. Conventional shift-invariant spaces correspond to the special case with identical generators, i.e., $g_{1}(x, y)=g_{2}(x, y)=\cdots=g_{M}(x, y)$. Similar to conventional shift-invariant spaces, we assume that the set $\bigcup_{m=1}^{M}\left\{g_{m}(x-k, y-l)\right\}_{(k, l) \in \mathcal{A} m}$ forms a Riesz basis for $V(\mathcal{G})$ in order to guarantee stability of (3.4).

For the time-varying fields considered in this chapter, we will assume that $h(x, y ; t) \in V(\mathcal{G})$ for all time instants $t$. Since we keep the generator functions constant over time, this means that the field coefficients $c_{k, l}$ 


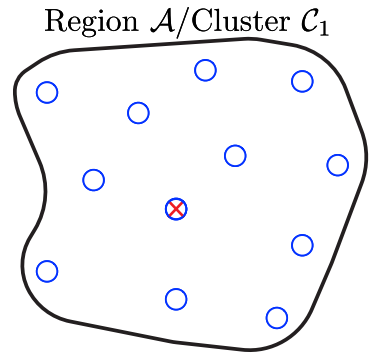

$\otimes \ldots$... Cluster Heads

(a)

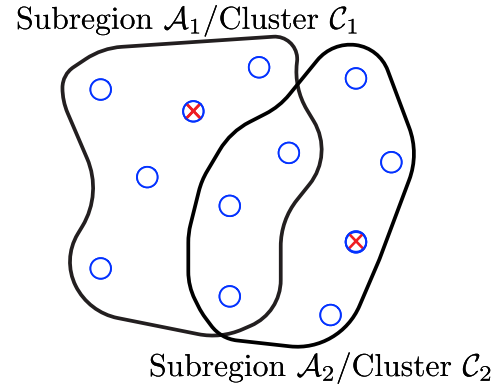

(b)

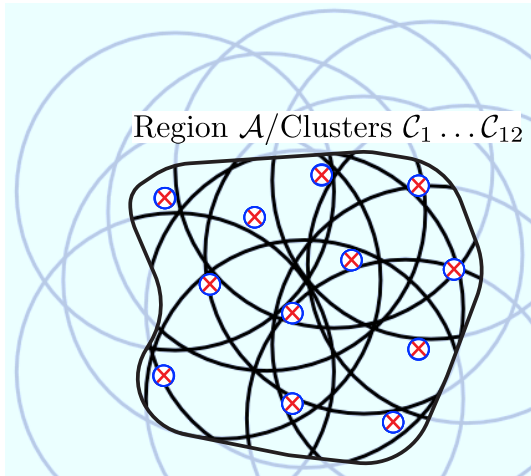

(c)

Figure 3.3: Clustering options for the proposed reconstruction scheme: (a) centralized setup with one cluster and a fusion center; (b) clustered architecture with several cluster heads; (c) diffusion-based reconstruction with every sensor node acting as cluster head.

in (3.4) become time-dependent. Even though we do not impose an explicit model on the temporal evolution of the coefficients $c_{k, l}(t)$, we will implicitly assume that their time-variation is slow.

\subsection{Field Reconstruction}

In the following sections, we describe three variants of our field reconstruction scheme that range from fully centralized to completely distributed and apply to different cluster granularities (i.e., one cluster, several clusters, $I$ clusters). The actual reconstruction algorithm is scalable and is identical for all three variants. However, these variants differ in how the computations are distributed among the sensor nodes and in the underlying communication protocol.

\subsubsection{Centralized Case - Global Reconstruction}

By extending [9] to two dimensions, we next show how the field $h(x, y ; n T) \in V(g)$ can be reconstructed within $\mathcal{A}$ from the $I$ (noisy) samples provided by the sensor nodes. In this section, we assume a centralized setup with one cluster consisting of all sensor nodes (see Figure 3.3(a)). Here, the cluster head serves as a global fusion center. We assume that the fusion center knows the measurements $h_{i}(n)$ and the positions $\left(x_{i}, y_{i}\right)$ of all sensor nodes as well as the generator function $g(x, y)$.

For every time instant $n T$, the fusion center first aims at determining least-squares (LS) estimates of the field coefficients by solving the minimization problem

$$
\hat{c}_{k, l}(n)=\arg \min _{c_{k, l}} \sum_{i=1}^{I}\left|\sum_{(k, l) \in \mathbb{A}} c_{k, l} g\left(x_{i}-k, y_{i}-l\right)-h_{i}(n)\right|^{2},
$$

where $\mathbb{A}=\mathbb{Z}^{2} \cap(\mathcal{A}+\mathcal{S})$. The problem (3.5) leads to a system of linear equations (see Section 3.5) which has a unique solution only if there are more sensor nodes (spatial samples) than unknown coefficients, i.e., 
$I \geq J \triangleq|\mathbb{A}|$. Once the LS coefficient estimates $\hat{c}_{k, l}(n)$ have been computed, the field within $\mathcal{A}$ can be reconstructed according to

$$
\hat{h}(x, y ; n T)=\sum_{(k, l) \in \mathbb{A}} \hat{c}_{k, l}(n) g(x-k, y-l)
$$

In this centralized setup, the sensor nodes send their measurements along with their positions to the fusion center that uses these data to solve the LS problem (3.5) and subsequently reconstruct the field according to (3.6). In Section 3.5, we will show that the compact support of the generator functions leads to extremely efficient solvers ${ }^{1}$.

The solution to the field reconstruction problem is initially known only to the fusion center, which is sufficient in many applications. For applications where the result has to be known throughout the WSN, the fusion center could communicate the solution of (3.5) to the sensor nodes which can then reconstruct the field (locally or globally). The centralized scheme requires a large amount of communication to transmit the data (directly or via multi-hop) to the fusion center. In exchange, the sensor nodes can be very simple (i.e., with low computational power) since they only need to take measurements and forward them to the fusion center. Obviously, a failure of the fusion center would tie up the operation of the whole sensor network. Moreover, if the sensor node placement does not result in a stable sampling set (e.g., too large gaps between some of the sensor nodes, see Section 3.6), the global reconstruction is likely to fail completely.

\subsubsection{Clustered Reconstruction}

We now consider the case of several clusters $(M>1)$, depicted in Figure 3.3(b), and we use a hybrid shiftinvariant space to model the unknown field, i.e, $h(x, y ; n T) \in V(\mathcal{G})$. In each cluster $\mathcal{C}_{m}$ there is a cluster head that performs local field reconstruction by using only the measurements and positions of the sensor nodes within that cluster. In order for local reconstruction within each subregion $\mathcal{A}_{m}$ to be feasible, we assume B-spline generator functions that have compact support $\mathcal{S}$. In this case, reconstruction within $\mathcal{A}_{m}$ only requires estimates of the coefficients $c_{k, l}(n)$ that lie within $\mathbb{A}_{m}=\mathbb{Z}^{2} \cap\left(\mathcal{A}_{m}+\mathcal{S}\right)$. These coefficients are estimated by solving the LS problem

$$
\hat{c}_{k, l}^{(m)}(n)=\arg \min _{c_{k, l}} \sum_{i \in \mathcal{C}_{m}}\left|\sum_{(k, l) \in \mathbb{A}_{m}} c_{k, l} b_{N(m)}\left(x_{i}-k, y_{i}-l\right)-h_{i}(n)\right|^{2} .
$$

Here, $b_{N(m)}(x, y)$ is a B-spline whose order $N(m)$ depends on the cluster index $m$. The corresponding system of linear equations (see below for details) has a unique solution only if $I_{m} \geq J_{m} \triangleq\left|\mathbb{A}_{m}\right|$, i.e., if there are more sensor nodes in the cluster than unknown coefficients in the associated region. We note that this condition tends to be harder to meet for larger $M$. For bandlimited field reconstruction, this condition can never be met due to the infinite support of the generator function $g_{\mathrm{BL}}(x, y)$, i.e., clustering and local reconstruction is impossible in this case. Hence, bandlimited fields necessitate the centralized architecture presented in Section 3.4.1, i.e., a single "cluster" comprising all sensor nodes.

\footnotetext{
${ }^{1}$ Reconstruction is possible also for generators that are not compactly supported but with significantly higher computational complexity.
} 
Using the optimum coefficients $\hat{c}_{k, l}^{(m)}(n)$ in (3.7), the field within $\mathcal{A}_{m}$ is reconstructed as

$$
\hat{h}(x, y ; n T)=\sum_{(k, l) \in \mathbb{A}_{m}} \hat{c}_{k, l}^{(m)}(n) b_{N(m)}(x-k, y-l), \quad \text { for }(x, y) \in \mathcal{A}_{m} .
$$

The cluster-based local reconstruction is similar to the global reconstruction scheme described in Section 3.4.1, the difference being that the reconstruction problem is divided into smaller parts that are solved independently by the individual clusters. We re-iterate that this clustering approach is only possible with generator functions $g_{m}(x, y)$ of compact support. This cluster-based scheme requires the sensor nodes to communicate their measurements to their cluster head who solves the LS problem (3.7) and reconstructs the field according to (3.8) within the subregion $\mathcal{A}_{m}$. The resulting solution to the field reconstruction problem in the subregions is known only to the respective cluster head. However, a reconstruction of the complete field is possible if the cluster heads communicate their local solutions to each other or to a fusion center. In case of overlapping subregions $\mathcal{A}_{m}$, several cluster heads compute estimates $\hat{c}_{k, l}^{(m)}(n)$ for a specific coefficient. A refined estimated of $c_{k, l}(n)$ can here be obtained by a subsequent averaging, described in more detail in the next section.

The clustered architecture further simplifies the use of different compactly supported generator functions in each cluster, e.g., B-splines of different order $N(m)$, according to the field model based on hybrid shiftinvariant spaces (see Section 3.3.3). This allows the model to be adapted to the local smoothness properties of the underlying field $h(x, y ; t)$. Furthermore, the clustered scheme reduces the communication requirements since only the sensor nodes within a cluster need to send their positions and measurements to their cluster head (i.e., fewer long-distance transmissions). Moreover, the reconstruction is more robust because the failure of a cluster head or a poor sensor node placement deteriorate only the result in the subregion $\mathcal{A}_{m}$ without affecting the reconstruction in the other subregions.

\subsubsection{Diffusion-based Reconstruction}

Increasing the number of clusters to the extreme case $M=I$ results in a fully distributed architecture in which each sensor node acts as cluster head for an associated subregion (see Figure 3.3(c)). The subregion corresponding to each cluster head has to be chosen larger than the support $\mathcal{S}$ of the generator function, which corresponds precisely to the region containing the coefficients $c_{k, l}(n)$ that directly influence the measurement of that sensor node. In its role as cluster head, every node estimates the field (within the generator function's support) by solving the LS problem (3.7) using the measurements of the sensor nodes within its corresponding subregion. Since the same coefficient $c_{k, l}$ is estimated by a multitude of sensor nodes, the influence of the measurement and quantization noise can be reduced by computing a refined estimated by averaging those initial estimates $\hat{c}_{k, l}^{(m)}(n)$ with $m$ such that $(k, l) \in\left(x_{m}, y_{m}\right)+\mathcal{S}$. The relevance and reliability of the individual estimates (both of which depend on the distance of the coefficient's position from the cluster heads) can be taken into account in the averaging scheme by an appropriate weighting.

The distributed averaging can be implemented using algorithms like randomized gossip [37], average consensus [39], and consensus propagation [38]. The sensor nodes can subsequently use the averaged coefficients to reconstruct the field according to (3.8) within its neighborhood. This two-stage approach can be viewed as a diffusion scheme, i.e., the sensor nodes calculate local updates for their estimates based on the information 
obtained from exchanging initial estimates with their neighbors. In this way, the observations of all sensor nodes diffuse through the whole network over time, regardless of the specific network topology.

\subsection{Implementation of Reconstruction Algorithm}

In this section, we describe how the solutions to the LS problem (3.7) can be obtained in an efficient manner (note hat (3.5) is a special case of (3.7) obtained with $M=1$ ). It will be seen that under the assumption of compactly supported generators, the computational complexity of the resulting algorithm scales linearly with the number of sensor nodes.

\subsubsection{Matrix-Vector Formulation}

We first reformulate the field reconstruction problem in terms of matrices and vectors. Since all clusters perform reconstruction independently of each other, it is sufficient to focus on one cluster, say cluster $m$. For simplicity, we restrict to $\mathrm{B}$-splines as generator functions and to rectangular subregions $\mathcal{A}_{m}$. Let $\left(k_{0}, l_{0}\right)$ and $\left(k_{1}, l_{1}\right)$ denote the index pair in the lower-left and upper-right corner of $\mathcal{A}_{m}$, respectively, such that the number of unknown coefficients within $\mathcal{A}_{m}$ equals $J_{m}=K\left(l_{1}-l_{0}+1\right)$ with $K \triangleq k_{1}-k_{0}+1$. We define the $I_{m} \times J_{m}$ matrix

$$
\left[\mathbf{G}^{(m)}\right]_{j, p} \triangleq b_{N(m)}\left(x_{i_{j}}-k_{p}, y_{i_{j}}-l_{p}\right)
$$

where $i_{j}, j=1, \ldots, I_{m}$, denotes the indices of the sensor nodes located in $\mathcal{A}_{m}$ (i.e., $\mathcal{C}_{m}=\left\{i_{1}, \ldots, i_{I_{m}}\right\}$ ), $k_{p}=k_{0}+((p-1) \bmod K)$, and $l_{p}=l_{0}+\left\lfloor\frac{p-1}{K}\right\rfloor$. Here, $\lfloor p\rfloor$ denotes the largest integer not larger than $p$. We emphasize that the compact support of $b_{N(m)}(x, y)$ implies that $\mathbf{G}^{(m)}$ is a sparse matrix whenever $\mathcal{A}_{m}$ is larger than $\mathcal{S}$. Specifically, $\left[\mathbf{G}^{(m)}\right]_{j, p}=b_{N(m)}\left(x_{i_{j}}-k_{p}, y_{i_{j}}-l_{p}\right) \neq 0$ only if $\left(x_{i_{j}}-k_{p}, y_{i_{j}}-l_{p}\right) \in \mathcal{S}$, which can happen for at most $\lfloor|\mathcal{S}|\rfloor=\lfloor N(m)+1\rfloor^{2}$ of the $J_{m}$ elements in each row. Thus, only a fraction of roughly $|\mathcal{S}| /\left|\mathcal{A}_{m}\right|$ of the elements in each row of $\mathbf{G}^{(m)}$ is non-zero.

Similar to (3.9), the measurements and unknown coefficients within the subregion under consideration are arranged into respective vectors $\mathbf{h}^{(m)}(n)$ and $\mathbf{c}^{(m)}(n)$, i.e.,

$$
\left[\mathbf{h}^{(m)}(n)\right]_{j} \triangleq h_{i_{j}}(n), \quad\left[\mathbf{c}^{(m)}(n)\right]_{p} \triangleq c_{k_{p}, l_{p}}^{(m)}(n) .
$$

With these definitions, we cast the minimization problem (3.5) as

$$
\hat{\mathbf{c}}^{(m)}(n)=\arg \min _{\mathbf{c}}\left\|\mathbf{G}^{(m)} \mathbf{c}-\mathbf{h}^{(m)}(n)\right\|^{2},
$$

whose solution is given by

$$
\hat{\mathbf{c}}^{(m)}(n)=\left(\mathbf{G}^{(m) H} \mathbf{G}^{(m)}\right)^{-1} \mathbf{G}^{(m) H} \mathbf{h}^{(m)}(n) .
$$

We note that this unique LS solution requires $\mathbf{G}^{(m)}$ to have full column rank which in turn presupposes $I_{m}>$ $J_{m}$ and guarantees the Gram matrix $\mathbf{G}^{(m) H} \mathbf{G}^{(m)}$ to be nonsingular (more specifically, positive definite). This is just another consequence of the requirement that there have to be enough appropriately spaced samples available. Technically, the sensor node positions $\left(x_{i_{j}}, y_{i_{j}}\right), i_{j} \in \mathcal{C}_{m}$, have to form a stable sampling set (see 
Section 3.6.2 for further details). Rather than computing $\hat{\mathbf{c}}^{(m)}(n)$ according to (3.12), it is advantageous to exploit the sparsity of $\mathbf{G}^{(m)}$ by solving the associated normal equations [45]:

$$
\mathbf{G}^{(m) H} \mathbf{G}^{(m)} \hat{\mathbf{c}}^{(m)}(n)=\mathbf{G}^{(m) H} \mathbf{h}^{(m)}(n) .
$$

Efficient direct and iterative solvers for these equations are discussed in Section 3.5.2.

\subsubsection{Efficient Solvers for the Sparse LS Problem}

In order to keep memory and complexity as low as possible, a solver for the normal equations (3.13) should capitalize on the sparsity of the matrix $\mathbf{G}^{(m)}$. Solving the normal equations by explicitly computing the Gram matrix $\mathbf{G}^{(m) H} \mathbf{G}^{(m)}$ is known to suffer from the squaring of the condition number of $\mathbf{G}^{(m)}$ [46].

An introduction to sparse direct solvers and an extensive numerical evaluation of different algorithms can be found in [47] and [48] respectively. In our context, an attractive method for solving (3.13) that exploits the sparsity of $\mathbf{G}^{(m)}$ and does not explicitly compute the Gram matrix is based on the sparse QR decomposition. Here, $\mathbf{G}^{(m)}$ is factored according to $\mathbf{G}^{(m)}=\mathbf{Q}^{(m)} \mathbf{R}^{(m)}$ where $\mathbf{Q}^{(m)}$ is an $I_{m} \times J_{m}$ matrix with orthonormal columns and $\mathbf{R}^{(m)}$ is a $J_{m} \times J_{m}$ upper triangular matrix. Using the fact that $\mathbf{G}^{(m) H} \mathbf{G}^{(m)}=\mathbf{R}^{(m) H} \mathbf{R}^{(m)}$ (the Cholesky factorization of the Gram matrix) and defining $\overline{\mathbf{h}}^{(m)}(n) \triangleq \mathbf{G}^{(m) H} \mathbf{h}^{(m)}(n)$, (3.13) can be rewritten as

$$
\mathbf{R}^{(m) H} \mathbf{R}^{(m)} \hat{\mathbf{c}}^{(m)}(n)=\overline{\mathbf{h}}^{(m)}(n),
$$

which can be solved efficiently by successively applying forward elimination and backward substitution, respectively, to the two triangular systems of equations

$$
\mathbf{R}^{(m) H} \tilde{\mathbf{c}}^{(m)}(n)=\overline{\mathbf{h}}^{(m)}(n), \quad \mathbf{R}^{(m)} \hat{\mathbf{c}}^{(m)}(n)=\tilde{\mathbf{c}}^{(m)}(n) .
$$

The complexity of this scheme will be shown below to scale linearly with the number of sensor nodes. The direct method is robust and provides an effective means for solving our field reconstruction problem for sensor node measurements from multiple time instances because the $\mathrm{QR}$ factorization needs to be performed only once (as long as the sensor node positions and therefore the matrix $\mathbf{G}^{(m)}$ do not change).

Iterative methods for solving the LS problems generate a sequence of approximate solutions $\hat{\mathbf{c}}_{q}^{(m)}(n), q=$ $1,2,3, \ldots$. The complexity of these schemes is dominated by the multiplication of certain intermediate vectors with the matrices $\mathbf{G}^{(m)}$ or $\mathbf{G}^{(m) H}$ and hence have the advantage of directly exploiting the sparsity of $\mathbf{G}^{(m)}$. The iterations are terminated either when a certain accuracy has been achieved or a prescribed maximum number of iterations is reached. The latter can have a regularizing effect which is desirable for highly irregular sensor node placements and noisy field measurements. Effective iterative methods for the LS problem in our context are Generalized Minimal RESidual method (GMRES) [49] and LSQR [50]. For time-varying fields, iterative methods have the additional advantage of being able to use the coefficient estimate from the previous time slot as initialization. This typically results in a significant reduction of the number of required iterations, in particular if the field does not change too fast between subsequent sampling instants. Iterative methods furthermore are better suited to the case of moving sensor nodes since here only an updated version of $\mathbf{G}^{(m)}$ has to be used without the need of repeatedly computing a QR factorization. 


\subsubsection{Algorithm Summary and Complexity}

In the following, we summarize all algorithmic steps necessary to perform field reconstruction and we provide estimates for their computational complexity.

\section{Preprocessing}

Iterative methods only require to determine the matrix $\mathbf{G}^{(m)}$ according to (3.9). Due to the sparse nature of $\mathbf{G}^{(m)}$, this requires at most $I_{m}(N(m)+1)^{2}$ function evaluations. The direct method proposed above (see 3.5.2), additionally requires a sparse $\mathrm{QR}$ factorization whose complexity scales as $\mathcal{O}\left(I_{m}(N(m)+1)^{4}\right)$ [45]. The computation of the matrix $\mathbf{G}^{(m)}$ and its sparse $\mathrm{QR}$ factorization needs to be done only once. Since it requires only the sensor node positions and the generator function to be known, these steps can be performed by the cluster head during the initialization phase of the WSN, i.e., before any measurements $h_{i}(n)$ are obtained.

\section{Coefficient Estimation}

Once measurements have been collected, the matrix-vector multiplications $\overline{\mathbf{h}}^{(m)}(n)=\mathbf{G}^{(m) H} \mathbf{h}^{(m)}(n)$ need to be performed. Due to the sparsity of $\mathbf{G}^{(m)}$, this calculation can be achieved with $\mathcal{O}\left(I_{m}(N(m)+1)^{2}\right)$ operations. With the iterative solvers, $\mathcal{O}\left(I_{m}(N(m)+1)^{2}\right)$ operations per iterations accrue from similar matrix-vector multiplications involving $\mathbf{G}^{(m)}$. With the direct solvers, the forward elimination and backward substitution require $\mathcal{O}\left(I_{m}(N(m)+1)^{2}\right)$ operations as well.

\section{Field Reconstruction}

The field can finally be reconstructed at any point $(x, y) \in \mathcal{A}_{m}$ according to (3.6). This requires $\mathcal{O}\left((N(m)+1)^{2}\right)$ operations per spatial point.

\section{Overall Complexity}

Adding up the operation counts for all clusters, we conclude that the overall complexity of our scheme scales as $\mathcal{O}\left(I N_{\max }^{4}\right)\left(N_{\max }\right.$ denotes the maximum spline order used), i.e., linearly with the number of sensor nodes and to the fourth power of the support area of the generator functions. Many small clusters appear desirable in order to distribute computations as much as possible and to reduce the communication requirements. With small clusters, however, the condition $I_{m} \geq J_{m}$ tends to be violated more often, possibly causing reconstruction to fail locally.

\subsection{Sensor Node Placement}

\subsubsection{Sensor Node Deployment}

The field reconstruction quality depends on the quality of the sensor node measurements, the number of sensors used and their placement. Here, we consider three different types of sensor node placement and their influence 


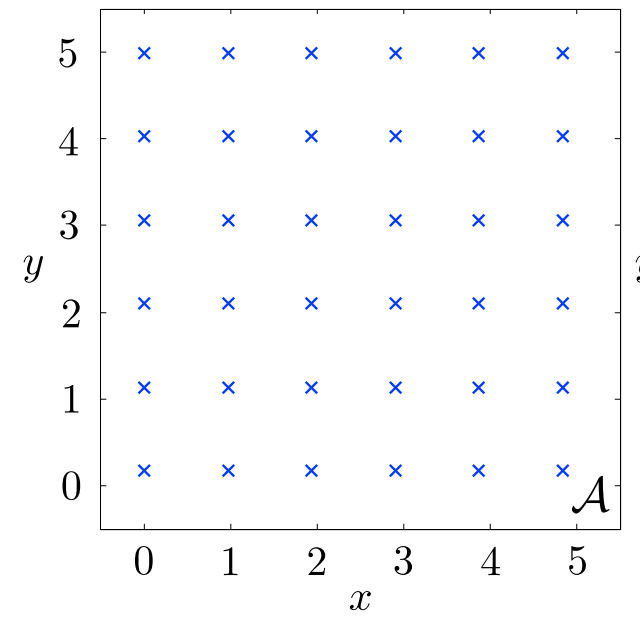

(a)

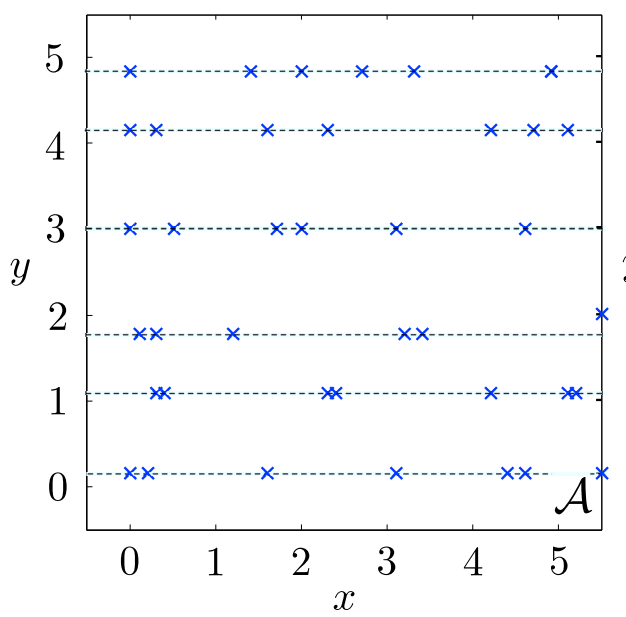

(c)

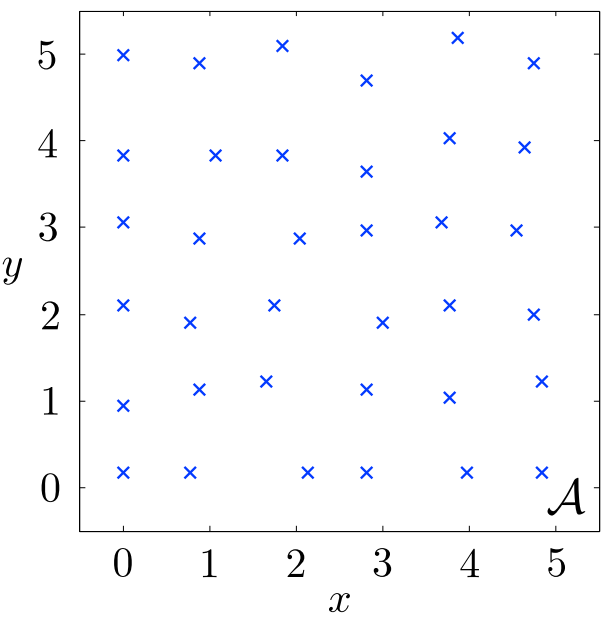

(b)

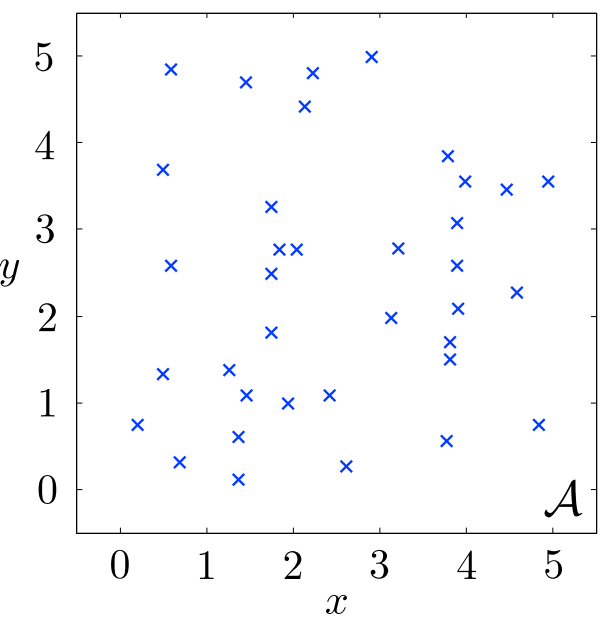

(d)

Figure 3.4: WSN deployments: (a) regular sensor placement, (b) 2-D jittered sensor placement, (c) 1-D jittered sensor placement (line sampling), (d) random sensor placement.

on the reconstruction algorithm and the quality of its results. The strictest requirements for the deployment of the sensor nodes are imposed by a uniform placement on the rectangular lattice (see Figure 3.4(a))

$$
\left(x_{i}, y_{i}\right) \in\left(\begin{array}{cc}
d_{x} & 0 \\
0 & d_{y}
\end{array}\right) \mathbb{Z}^{2} .
$$

As will be seen later, such a deployment is desirable performance-wise. A less demanding deployment corresponds to a jittered version of a rectangular spatial lattice (see Figure 3.4(b)), i.e.,

$$
\left(x_{i}-\lambda_{x, i}, y_{i}-\lambda_{y, i}\right) \in\left(\begin{array}{cc}
d_{x} & 0 \\
0 & d_{y}
\end{array}\right) \mathbb{Z}^{2},
$$

where $\left(\lambda_{x, i}, \lambda_{y, i}\right)$ is a random 2-D position jitter (e.g., uniformly distributed within $\left[-d_{x} / 4, d_{x} / 4\right] \times\left[-d_{y} / 4\right.$, $\left.d_{y} / 4\right]$ ). If the sensor nodes are placed on lines on one spatial dimension, we obtain the special case of so-called 
line sampling (see Figure 3.4(c)) [4]. Further relaxation of the positioning requirements will be modeled by a uniformly distributed random placement of the sensor nodes within $\mathcal{A}$ (see Figure 3.4(d)), corresponding to a 2-D homogeneous Poisson point process.

\subsubsection{Stable Sets of Sampling}

The feasibility and accuracy of the LS field reconstruction problem (3.5) depend crucially on the rank and condition number of the matrix G. Technically, the sensor node positions need to form a stable sampling set. We say that a set of points $\left\{\left(x_{i}, y_{i}\right)\right\}_{i=1, \ldots, I}$ is a set of stable sampling for the shift-invariant space $V(g)$ if there exist positive constants $A_{1}, A_{2}>0$, such that

$$
A_{1}\|f\|_{2}^{2} \leq \sum_{i=1}^{I}\left|f\left(x_{i}, y_{i}\right)\right|^{2} \leq A_{2}\|f\|_{2}^{2} \quad \text { for all } f \in V(g) .
$$

When restricted to the finite-dimensional subspace of functions $f(x, y)=\sum_{(k, l) \in \mathbb{A}} c_{k, l} g(x-k, y-l)$, then the above inequality implies that the condition number of the matrix $\mathbf{G}$ is upper bounded by $\sqrt{A_{2} / A_{1}}$.

For the 1-D case and for B-spline generators, stable sampling sets are well understood (see, e.g., [9,51]). For the 2-D case, the stability is a difficult mathematical problem whose general solution is still unknown. Our numerical simulations indicate that $\mathbf{G}$ is well-conditioned whenever the sensor node positions are well distributed in each cluster. In the following we provide some mathematical guarantees that support these numerical observations.

We first formulate a deterministic result for line sampling [4] in a 2-D shift-invariant space with a spline generator $b_{N}(x, y)$. We assume that the sensor node positions are distributed along lines at points $\left(x_{j}, y_{j, k}\right) \in$ $\mathbb{R}^{2}$ with $j, k \in \mathbb{Z}$, such that $x_{j}<x_{j+1}$ and $y_{j, k}<y_{j, k+1}$. Note that the sensor nodes may be distributed non-uniformly on each line and the lines themselves may be non-uniformly spaced. The proof of the following theorem is given in Section 3.A.

Theorem 1. Suppose that $d_{x} \triangleq \sup _{j \in \mathbb{Z}}\left(x_{j+1}-x_{j}\right)<1$ and $d_{y} \triangleq \sup _{j, k \in \mathbb{Z}}\left(y_{j, k+1}-y_{j, k}\right)<1$. Then the set $\left(x_{j}, y_{j, k}\right) \in \mathbb{R}^{2}$ is a set of stable sampling for $V\left(b_{N}\right)$.

Observe that the case of line sampling includes uniform rectangular sensor node placement as special case. The theorem therefore describes the minimum sampling density required for field reconstruction to be feasible. We note that previously existing qualitative results guaranteed the stability only with (unspecified) large oversampling factor (e.g., [52]).

For highly non-uniform sampling, even in the bandlimited case only probabilistic statements are available. The following result from [53, Thm. 6.1] estimates the condition number of $\mathbf{G}$ for the case of bandlimited reconstruction.

Theorem 2. Let $\left(x_{j}, y_{j}\right) \in \mathbb{R}^{2}$ be independent identically distributed random variables that are uniformly distributed over $\mathcal{A}$. Then there exist constants $a, b, c>0$, such that for all $\mu \in(0,1)$ the condition number of the matrix $\mathbf{G}$ is upper bounded by $\frac{(1+\mu)}{(1-\mu)}$ with probability at least $1-a e^{-b I \frac{\mu^{2}}{1+\mu}}$.

This result confirms the intuition that the reconstruction tends to become more and more stable as the number $I$ of sensor nodes increases. 


\subsubsection{Selective Dimension Reduction}

The Least Squares (LS) solution (3.12) includes the pseudo-inverse $\mathbf{G}^{(m) \#}=\left(\mathbf{G}^{(m) H} \mathbf{G}^{(m)}\right)^{-1} \mathbf{G}^{(m) H}$ of the matrix $\mathbf{G}^{(m)}$. Calculation of the pseudo-inverse involves the inversion of $\mathbf{G}^{(m) H} \mathbf{G}^{(m)}$, which is the Gram matrix of the column vectors of $\mathbf{G}^{(m)}$. The inversion of the matrix causes problems when $\mathbf{G}^{(m) H} \mathbf{G}^{(m)}$ is close to singularity, i.e., if $\operatorname{det}\left(\mathbf{G}^{(m) H} \mathbf{G}^{(m)}\right)$ is almost zero. Since $\mathbf{G}^{(m) H} \mathbf{G}^{(m)}$ is a positive semidefinite matrix its determinant can be upper bounded via Hadamard's inequality as

$$
\operatorname{det}\left(\mathbf{G}^{(m) H} \mathbf{G}^{(m)}\right) \leq \prod_{i=1}^{J_{m}}\left[\mathbf{G}^{(m) H_{\mathbf{G}}} \mathbf{G}^{(m)}\right]_{i i}=\prod_{i=1}^{J_{m}}\left\|\mathbf{f}_{i}\right\|^{2},
$$

where $\mathbf{f}_{i}$ are the column vectors of the matrix. To help prevent $\mathbf{G}^{(m) H} \mathbf{G}^{(m)}$ from being singular one can delete those columns $\mathbf{f}_{i}$ that have zero norm. Zero norm of a column implies that we don't have any information about the corresponding coefficient because no sensor lies within an area of the size of the support $\mathcal{S}$ around the coefficient's position. Also, for ill-conditioned matrices, removing columns $\mathbf{f}_{i}$ with small norm helps to improve the condition number of the Gram matrix $\mathbf{G}^{(m) H} \mathbf{G}^{(m)}$. Deleting columns corresponds to excluding the corresponding coefficients from the estimation/reconstruction process, thereby selectively reducing the dimension of the system of linear equations and consequently the complexity of solving it.

\subsubsection{Sensor Node Localization Error}

We next investigate the impact of sensor node localization errors in the absence of other impairments, restricting for simplicity to the 1-D case. While the actual sensor node positions equal $x_{i}$, the sensors report estimated positions $\tilde{x}_{i}$ to the cluster heads. Due to localization errors, $\tilde{x}_{i}$ differs from $x_{i}$. We assume that the localization errors $\delta_{i} \triangleq \tilde{x}_{i}-x_{i}$ are independent and uniformly distributed between $-\Delta_{0}$ and $\Delta_{0}$. For notational convenience, we drop the time index $n$ and the cluster index $m$ and assume that the actual field is given by $h(x)=\mathbf{c}^{T} \mathbf{g}(x)$ where $\mathbf{g}(x)=(g(x-k))_{k \in \mathbb{Z} \cap \mathcal{A}}^{T}$ and $\mathbf{c}$ is the vector with the true field coefficients. The latter are assumed to be i.i.d. with zero mean and variance $\sigma_{c}^{2}$.

The reconstructed field is given by $\hat{h}(x)=\hat{\mathbf{c}}^{T} \mathbf{g}(x)$ where $\hat{\mathbf{c}}$ denotes the coefficients estimated according to (the 1-D version of) (3.11) with the measurements given by $\tilde{h}_{i}=h\left(\tilde{x}_{i}\right)=h\left(x_{i}+\delta_{i}\right)$. Clearly, sensor node localization errors cause a mismatch between the sensor node positions and the sensor node measurements used for the reconstruction. Since the measurement value originates from a different position it comprises a measurement error that depends on the sensor node localization error. The mean-square reconstruction error within $\mathcal{A}$ can now be developed as

$$
\begin{aligned}
\varepsilon & \triangleq \mathrm{E}\left\{\int_{\mathcal{A}}(\hat{h}(x)-h(x))^{2} d x\right\}=\mathrm{E}\left\{\int_{\mathcal{A}}\left((\hat{\mathbf{c}}-\mathbf{c})^{T} \mathbf{g}(x)\right)^{2} d x\right\} \\
& =\int_{\mathcal{A}} \mathbf{g}^{T}(x) \mathrm{E}\left\{(\hat{\mathbf{c}}-\mathbf{c})(\hat{\mathbf{c}}-\mathbf{c})^{T}\right\} \mathbf{g}(x) d x=\int_{\mathcal{A}} \operatorname{tr}\left\{\mathbf{C}_{\hat{\mathbf{c}}-\mathbf{c}} \mathbf{g}(x) \mathbf{g}^{T}(x)\right\} d x \\
& =\operatorname{tr}\left\{\mathbf{C}_{\hat{\mathbf{c}}-\mathbf{c}} \int_{\mathcal{A}} \mathbf{g}(x) \mathbf{g}^{T}(x) d x\right\}=\operatorname{tr}\left\{\mathbf{C}_{\hat{\mathbf{c}}-\mathbf{c}} \mathbf{G}_{\mathbf{g}}\right\},
\end{aligned}
$$


where the expectation is with respect to the localization errors $\delta_{i}$ and the field coefficients $\mathbf{c}$. In the last expression, we introduced the Gramian $\mathbf{G}_{\mathbf{g}}=\int_{\mathcal{A}} \mathbf{g}(x) \mathbf{g}^{T}(x) d x$ and the matrix $\mathbf{C}_{\hat{\mathbf{c}}-\mathbf{c}}=\mathrm{E}\left\{(\hat{\mathbf{c}}-\mathbf{c})(\hat{\mathbf{c}}-\mathbf{c})^{T}\right\}$ which is the correlation matrix of the coefficient errors $\hat{\mathbf{c}}-\mathbf{c}$. The Gramian $\mathbf{G}_{\mathbf{g}}$ can easily be shown to be a symmetric banded Toeplitz matrix (the band structure results from the compact support of the B-spline generator functions). Since the coefficient error can be written as (see (3.11))

$$
\hat{\mathbf{c}}-\mathbf{c}=\mathbf{G}^{\#} \tilde{\mathbf{h}}-\mathbf{G}^{\#} \mathbf{h}=\mathbf{G}^{\#} \mathbf{e} \quad \text { with } \mathbf{e}=\tilde{\mathbf{h}}-\mathbf{h},
$$

its correlation matrix equals

$$
\mathbf{C}_{\hat{\mathbf{c}}-\mathbf{c}}=\mathbf{G}^{\#} \mathbf{C}_{\mathbf{e}}\left(\mathbf{G}^{\#}\right)^{T}, \quad \text { where } \mathbf{C}_{\mathbf{e}}=\mathrm{E}\left\{\mathbf{e} \mathbf{e}^{T}\right\} .
$$

Here, $\tilde{\mathbf{h}}$ and $\mathbf{h}$ are vectors that aggregate $\tilde{h}_{i}$ and $h_{i}$, respectively. The element $e_{i}$ of e, i.e., the measurement error at sensor node $i$ caused by the localization error $\delta_{i}$, reads

$$
e_{i}=\tilde{h}_{i}-h_{i}=\sum_{k} c_{k} \Delta_{g}\left(x_{i}-k, \delta_{i}\right)
$$

where $\Delta_{g}(x, \delta)=g(x+\delta)-g(x)$. Using the statistical independence of the field coefficients $c_{k}$ and the localization error $\delta_{i}$, it follows that the $(i, j)$ th element of $\mathbf{C}_{\mathbf{e}}$ equals

$$
\begin{aligned}
{\left[\mathbf{C}_{\mathbf{e}}\right]_{i, j} } & =\mathrm{E}\left\{e_{i} e_{j}\right\} \\
& = \begin{cases}\sigma_{c}^{2} \sum_{k} \mathrm{E}\left\{\Delta_{g}^{2}\left(x_{i}-k, \delta_{i}\right)\right\}, & i=j, \\
\sigma_{c}^{2} \sum_{k} \mathrm{E}\left\{\Delta_{g}\left(x_{i}-k, \delta_{i}\right)\right\} \mathrm{E}\left\{\Delta_{g}\left(x_{j}-k, \delta_{j}\right)\right\}, & i \neq j,\end{cases}
\end{aligned}
$$

where the expectation now is with respect to the location error $\delta_{i}$ only.

By specializing the above expressions for a given generator, the mean square reconstruction error can be calculated for a given WSN deployment. As an example, we provide the results for a B-spline of order $N=1$, i.e.,

$$
\tilde{b}_{1}(x)= \begin{cases}1-|x|, & |x| \leq 1, \\ 0, & \text { else. }\end{cases}
$$

Here, the first row of the symmetric Toeplitz matrix $\mathbf{G}_{g}$ equals

$$
\left[\int_{\mathcal{A}} \tilde{b}_{1}^{2}(x) d x \quad \int_{\mathcal{A}} \tilde{b}_{1}(x) \tilde{b}_{1}(x-1) d x \quad 0 \quad \ldots \quad 0\right]=\left[\begin{array}{lllll}
\frac{2}{3} & \frac{1}{6} & 0 & \ldots & 0
\end{array}\right] .
$$

Furthermore, denoting the integer closest to $x_{i}$ by $k_{i}$ and the distance of $x_{i}$ from the closest integer by $\bar{x}_{i}=$ $k_{i}-x_{i}$, the (normalized) variance of the field measurement error is obtained as

$$
\frac{\mathrm{E}\left\{e_{i}^{2}\right\}}{\sigma_{c}^{2}}= \begin{cases}\frac{2 \Delta_{0}^{2}}{3} & \left|\bar{x}_{i}\right| \geq \Delta_{0}, \\ \frac{2 \Delta_{0}^{2}}{3}-\frac{3 \Delta_{0}\left|\bar{x}_{i}\right|}{2}+3 \bar{x}_{i}^{2}-\frac{3\left|\bar{x}_{i}\right|^{3}}{2 \Delta_{0}} & \left|\bar{x}_{i}\right|<\Delta_{0},\end{cases}
$$

and the correlations equal

$$
\mathrm{E}\left\{e_{i} e_{j}\right\}=\rho_{i j} \sigma_{c}^{2} p\left(\bar{x}_{i}\right) p\left(\bar{x}_{j}\right)
$$


with

$$
p(z)= \begin{cases}\frac{z^{2}}{4 \Delta_{0}}-\frac{|z|}{2}+\frac{\Delta_{0}}{4} & |z|<\Delta_{0}, \\ 0 & \text { else. }\end{cases}
$$

Here, the coefficients $\rho_{i j}$ are given by

$$
\rho_{i j}=\left\{\begin{aligned}
6, & k_{i}=k_{j} \\
-4, & \left|k_{i}-k_{j}\right|=1 \\
1, & \left|k_{i}-k_{j}\right|=2 \\
0, & \left|k_{i}-k_{j}\right| \geq 3
\end{aligned}\right.
$$

The derived formulae (3.14) and (3.15) allow, together with the results for $\mathbf{G}_{\mathbf{g}}$ and $\mathbf{C}_{\mathbf{e}}$ (e.g., (3.16), (3.17), and (3.18) when using a B-spline of order $N=1$ ), to calculate the MSE of a reconstructed field for a deployed sensor network, where the sensor node positions are known but impaired by a jitter of maximum value $\Delta_{0}$.

\subsection{Numerical Simulations}

We present numerical results to illustrate the performance of our distributed sampling and reconstruction scheme. We verify the suitability of hybrid shift-invariant spaces as field models for non-bandlimited physical fields by considering diffusion fields over the region $\mathcal{A}=[0,20] \times[0,20]$. Figure 3.5 shows an example for such a diffusion field and illustrates the partitioning of the region $\mathcal{A}$ into four disjoint square subregions $\mathcal{A}_{m}$ of equal size with corresponding sensor node clusters $\mathcal{C}_{m}$. The diffusion field was generated by $P$ sources whose strength, position, and activation time are denoted by $A_{p},\left(x_{p}, y_{p}\right)$, and $t_{p}$, respectively. The resulting 2-D diffusion field can then be written as

$$
h(x, y ; t)=\sum_{p=1}^{P} A_{p} K\left(x-x_{p}, y-y_{p} ; t-t_{p}\right)
$$

with the heat kernel

$$
K(x, y ; t)=H(t) \frac{1}{4 \pi \kappa t} \mathrm{e}^{-\frac{x^{2}+y^{2}}{4 \kappa t}} .
$$

Here, $H(t)$ denotes the unit step function which equals 1 for $t \geq 0$ and 0 for $t<0$, and $\kappa$ is the diffusion coefficient (e.g., thermal conductivity in the case of a temperature field).

In the following, unless stated otherwise, we used source positions $\left(x_{p}, y_{p}\right)$ uniformly distributed over $\mathcal{A}$, amplitudes $A_{p}$ uniformly distributed in the interval $[-10,10]$, and $\kappa=0.1$. For the bandlimited reconstruction we use the generator function in (3.1) with spatial bandwidths $B_{x}=B_{y}=1$, while for the non-bandlimited reconstruction we use our clustered scheme with B-splines of order $N=0, \ldots, 8$. The average sensor node density will be denoted by $D \triangleq \frac{I}{|\mathcal{A}|}$. The quality of the field reconstruction will be measured in terms of the normalized Mean Square Error (MSE)

$$
\varepsilon=\frac{\mathrm{E}\left\{\|\hat{h}-h\|^{2}\right\}}{\mathrm{E}\left\{\|h\|^{2}\right\}}
$$

which is empirically estimated using Monte Carlo simulations. 


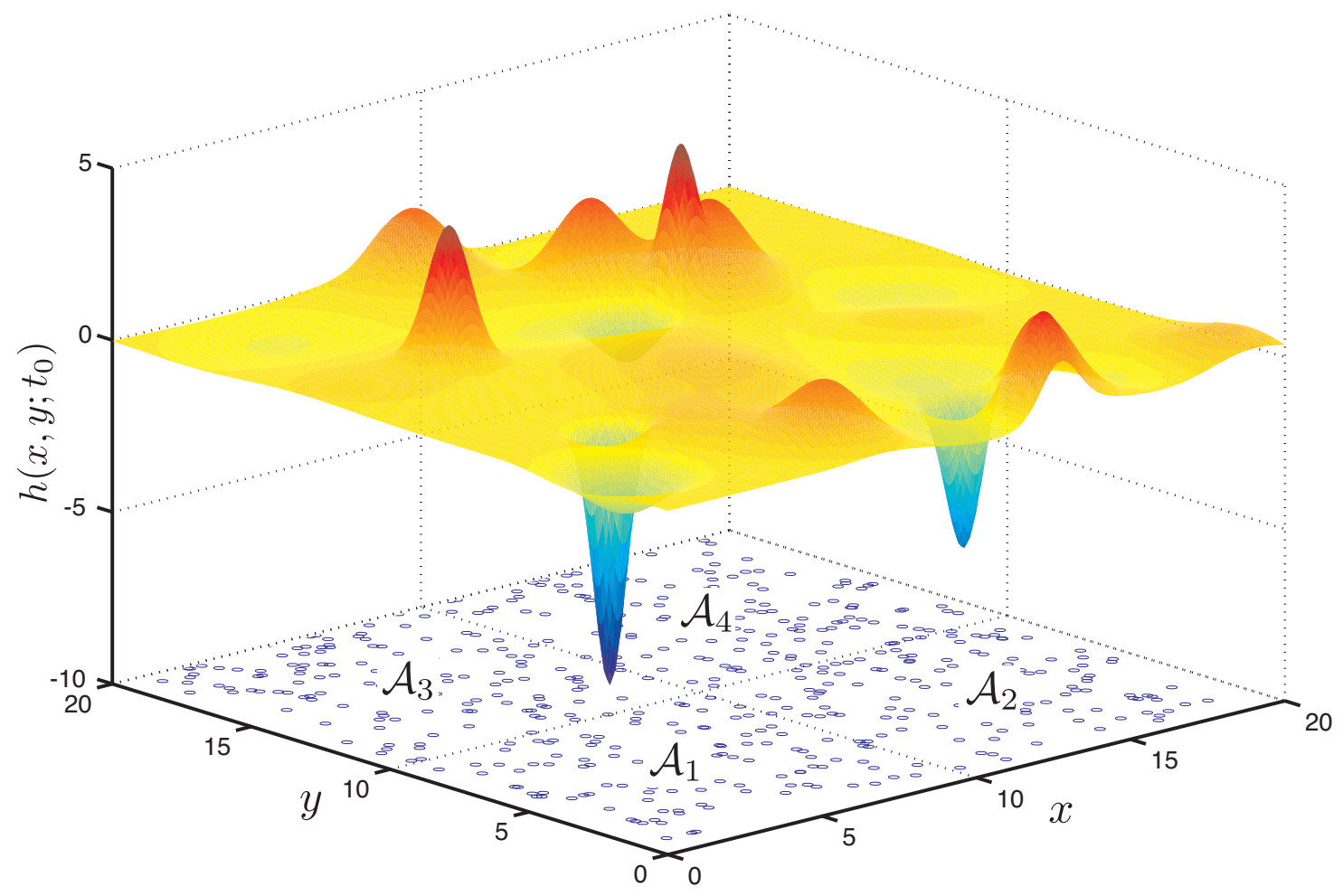

Figure 3.5: Example for a diffusion field $h\left(x, y ; t_{0}\right)$ and illustration of the partitioning of the region $\mathcal{A}$ into four disjoint square subregions $\mathcal{A}_{1}, \ldots, \mathcal{A}_{4}$ of equal size and of the sensor nodes (drawn as circles) into corresponding clusters $\mathcal{C}_{1}, \ldots, \mathcal{C}_{4}$.

\subsubsection{Comparison of Bandlimited Reconstruction with Reconstruction using B- Splines}

We first compare the bandlimited reconstruction with our reconstruction scheme using B-splines for the static case (we drop the time dependence for the static setup for sake of notational simplicity). Here, the spline order was optimized either locally or globally. We used $P=40$ sources activated at time instants that were Poisson distributed in the interval $[-10 T,-T]$. The field was reconstructed from non-quantized sensor node measurements impaired by measurement noise only. We compare the reconstruction results for the sensor node placements described in Section 3.6.1.

Figure 3.6 shows the results for $M=4$ and $M=16$ identical but disjoint square subregions $\mathcal{A}_{m}$ for the case of no measurement noise. Figure 3.7 shows the results for the same setup but with a measurement SNR of $15 \mathrm{~dB}$. For both cases, it can be seen in all subfigures that a higher sensor node density is advantageous for the reconstruction quality. A higher sensor node density assures a better estimation of the coefficients and therefore a better reconstruction of the entire field. Whereas in the noiseless case with regular sensor node placement (see Figure 3.6(a)) only little gains can be achieved by increasing the sensor node density, in the case of jittered (see 


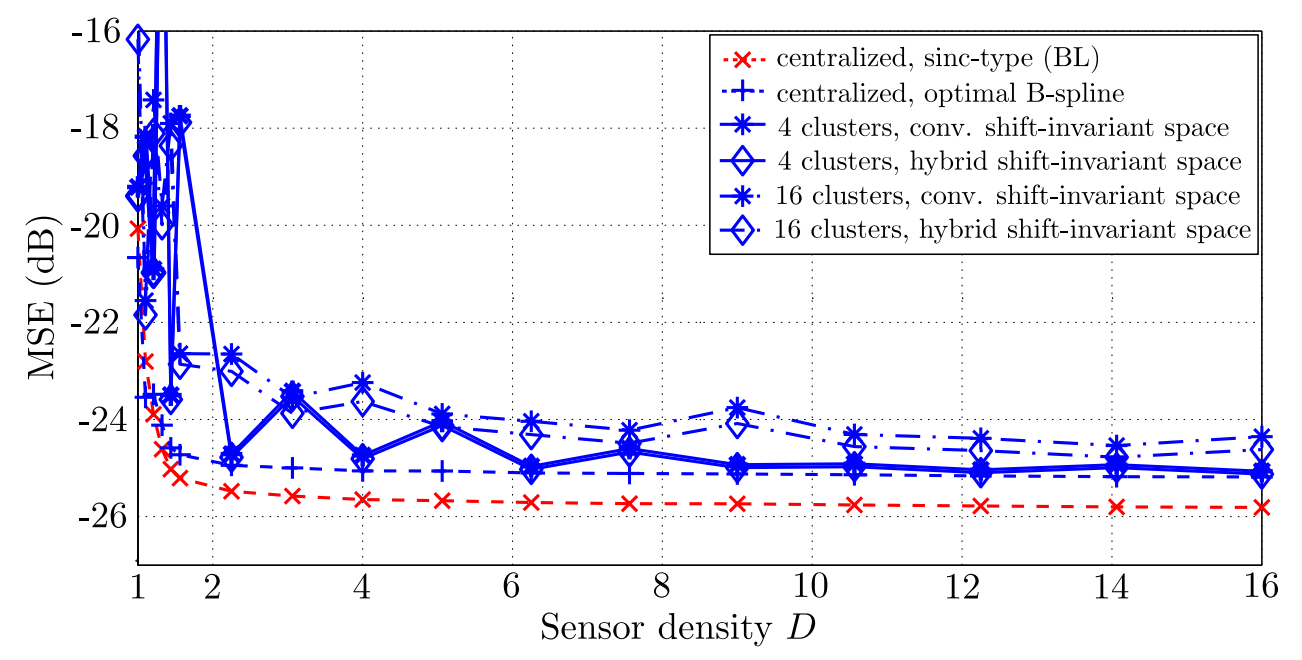

(a)

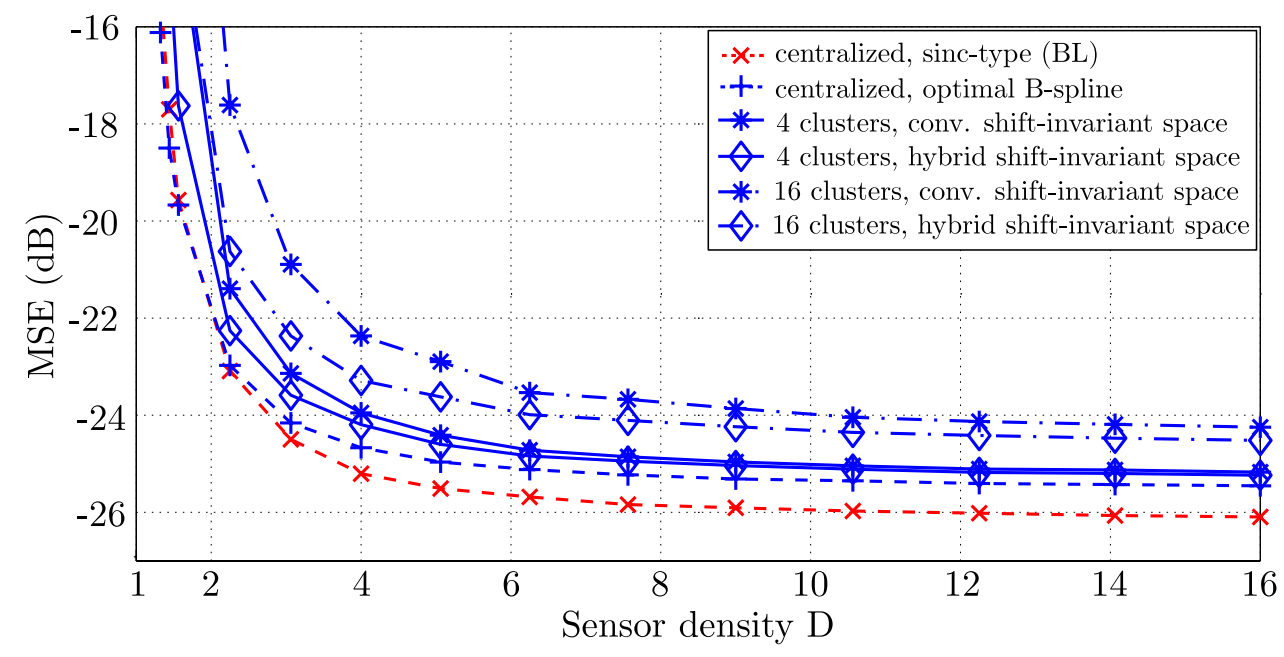

(b)

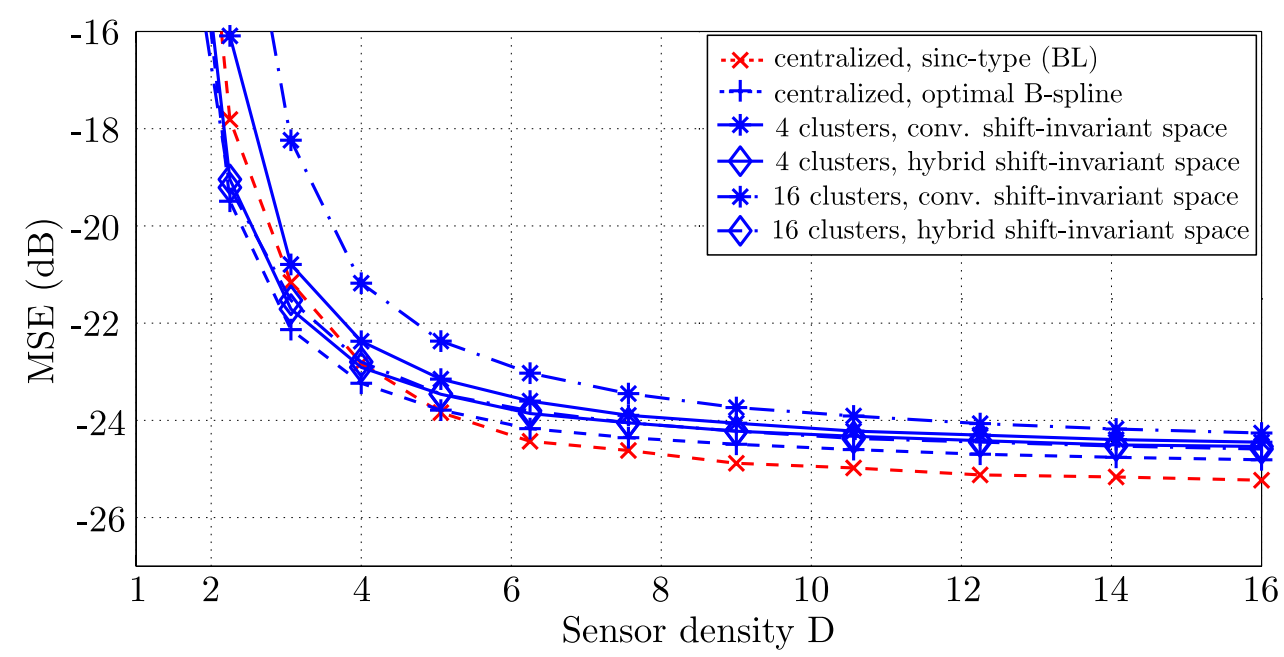

(c)

Figure 3.6: Reconstruction of diffusion fields: MSE versus sensor node density achieved with centralized bandlimited reconstruction, centralized B-spline reconstruction, and clustered B-spline reconstruction using 4 and 16 non-overlapping subregions for the case of no measurement noise: (a) sensor node placement on square lattice, (b) square lattice with jitter, (c) random sensor node placement. 


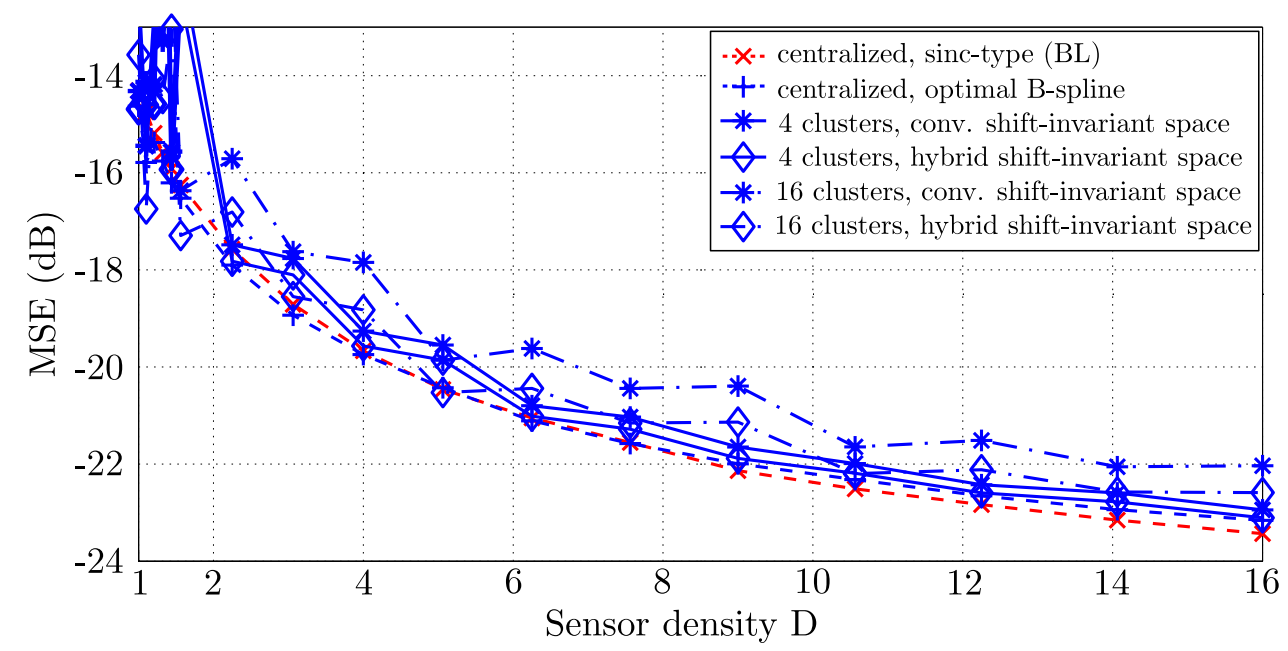

(a)

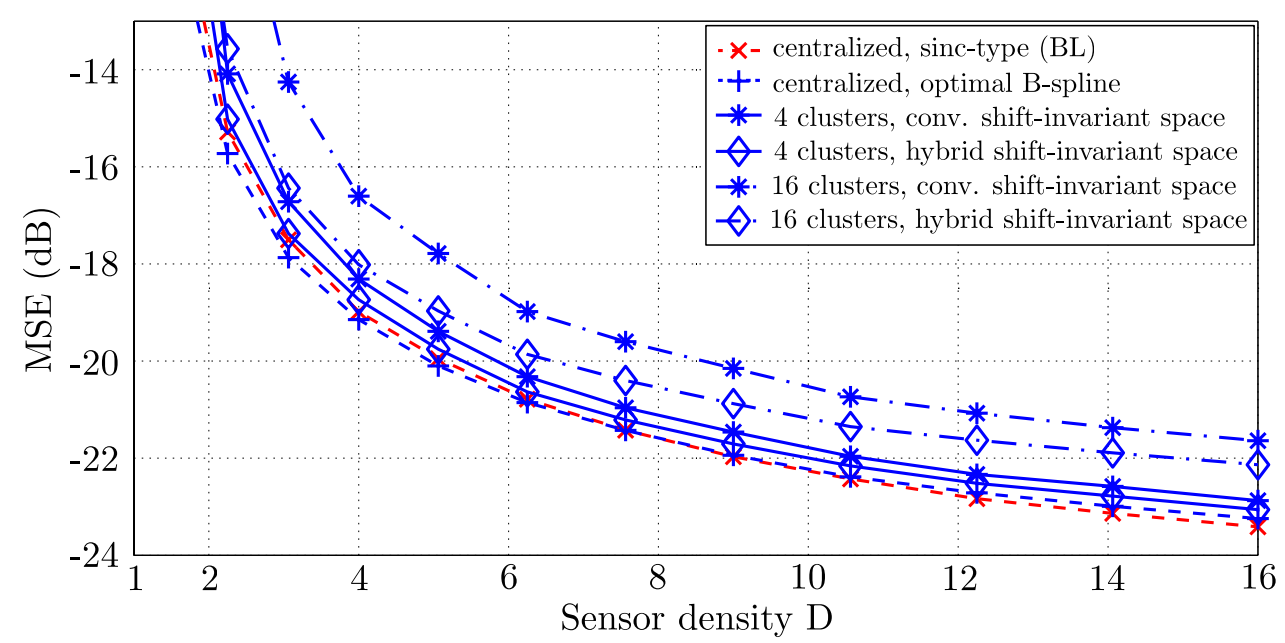

(b)

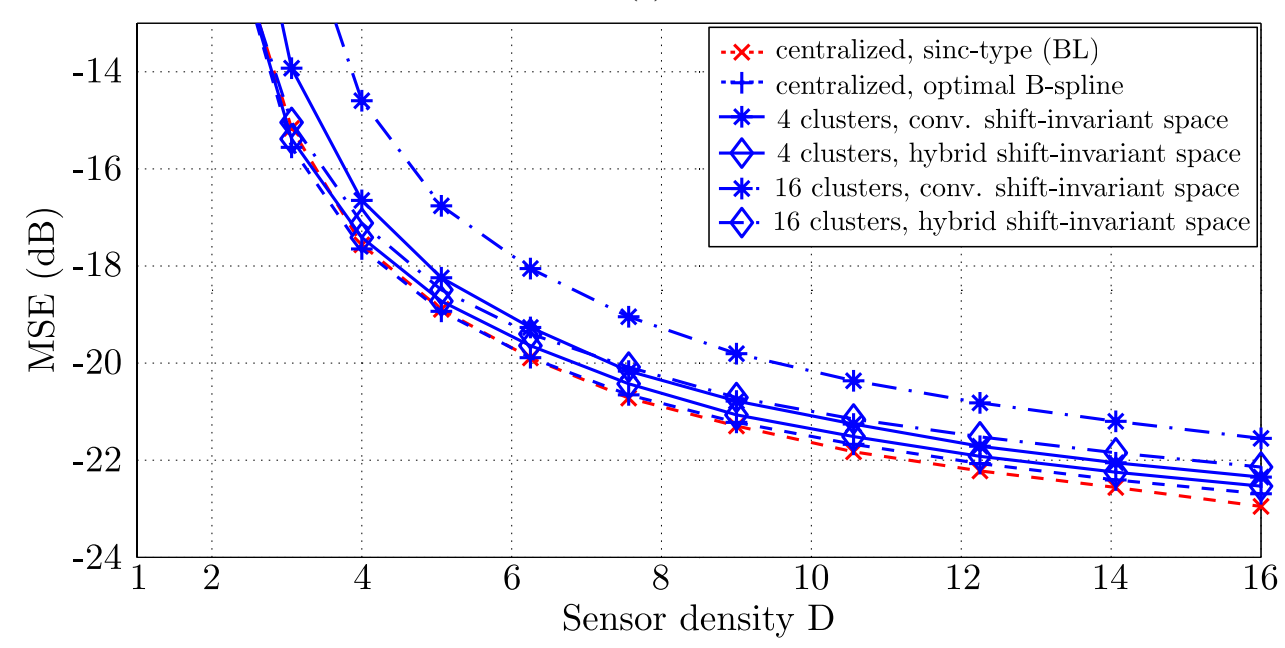

(c)

Figure 3.7: Reconstruction of diffusion fields: MSE versus sensor node density achieved with centralized bandlimited reconstruction, centralized B-spline reconstruction, and clustered B-spline reconstruction using 4 and 16 non-overlapping subregions for $15 \mathrm{~dB}$ measurement SNR: (a) sensor node placement on square lattice, (b) square lattice with jitter, (c) random sensor node placement. 
Figure 3.6(b)) and random sensor node placement (see Figure 3.6(c)) higher sensor node density results in a smaller distance between neighboring sensor nodes and therefore better reconstruction quality. In the presence of measurement noise (see Figure 3.7), a larger number of sensor nodes additionally helps to average out the influence of the noise.

It can be seen in all subfigures of Figures 3.6 and 3.7, that in these scenarios bandlimited reconstruction achieves only slightly better reconstruction quality than B-splines at the cost of much higher computational complexity. Introducing clustering (4 and 16 clusters respectively) leads to a penalty in the reconstruction, where the penalty gets larger for a higher number of clusters. The penalty can be partly tackled by allowing reconstruction in hybrid shift-invariant spaces, i.e., by allowing the use of different spline orders for reconstruction within the clusters. We compare reconstruction in hybrid shift-invariant spaces using the locally optimal spline order for each cluster and reconstruction in conventional shift-invariant spaces using the globally optimal spline order for all clusters, where the spline orders were optimized empirically. Different spline orders within the clusters allow to better adapt to the local smoothness properties of the field and therefore leads to a lower reconstruction error, which is reflected in Figures 3.6 and 3.7.

In the examples above the bandlimited reconstruction shows slightly better reconstruction quality compared to reconstruction in shift-invariant spaces using B-splines. This is not the case in general and strongly depends of the type of the underlying field to be reconstructed. In particular, if we consider source fields constructed as elements of a shift-invariant space $V(\bar{g})$ induced by a Gaussian generator function, i.e.,

$$
\bar{g}(x, y)=\mathrm{e}^{-\lambda^{2}\left(x^{2}+y^{2}\right)},
$$

our reconstruction scheme outperforms the bandlimited reconstruction not only in complexity but also in reconstruction quality. Note that the elements of the space $V(\bar{g})$ are as well diffusion fields with sources that are positioned on the integer lattice and that are simultaneously activated. Figure 3.8 shows the results using a Gaussian generator function $\bar{g}(x, y)$ with $\lambda=1.33$ and i.i.d. normally distributed field coefficients in absence of measurement noise. This example shows the dependence of the reconstruction quality of the two compared reconstruction schemes on the underlying field. However, the reconstruction scheme based on shift-invariant spaces using B-splines as generator functions provides lower reconstruction complexity in any case and moreover allows the overall reconstruction problem to be partitioned into smaller problems.

\subsubsection{Averaging of Coefficients}

To illustrate the gains possible by averaging the coefficient estimates from different overlapping clusters we used a simple setup with $M=2$ overlapping subregions $\mathcal{A}_{1}=\left[0, \frac{40}{3}\right] \times[0,20], \mathcal{A}_{2}=\left[\frac{20}{3}, 20\right] \times[0,20]$ with respective sensor node clusters $\mathcal{C}_{1}$ and $\mathcal{C}_{2}$. The field coefficients for the two subregions were estimated independently by the two clusters using B-splines of order $N=3$. Afterwards those coefficients in the overlapping area that influence the field only in this area were averaged. In our example, this amounts to 63 (out of 441) coefficients being averaged (roughly 15\%). The resulting averaged coefficients are then used to reconstruct the field. Figure 3.9 shows the gain that can be obtained by averaging the coefficient estimates without measurement noise. It can be seen that for all sensor node placements, averaging of coefficient estimates between clusters leads to lower reconstruction errors than simply using the estimates of one cluster for the overlapping 


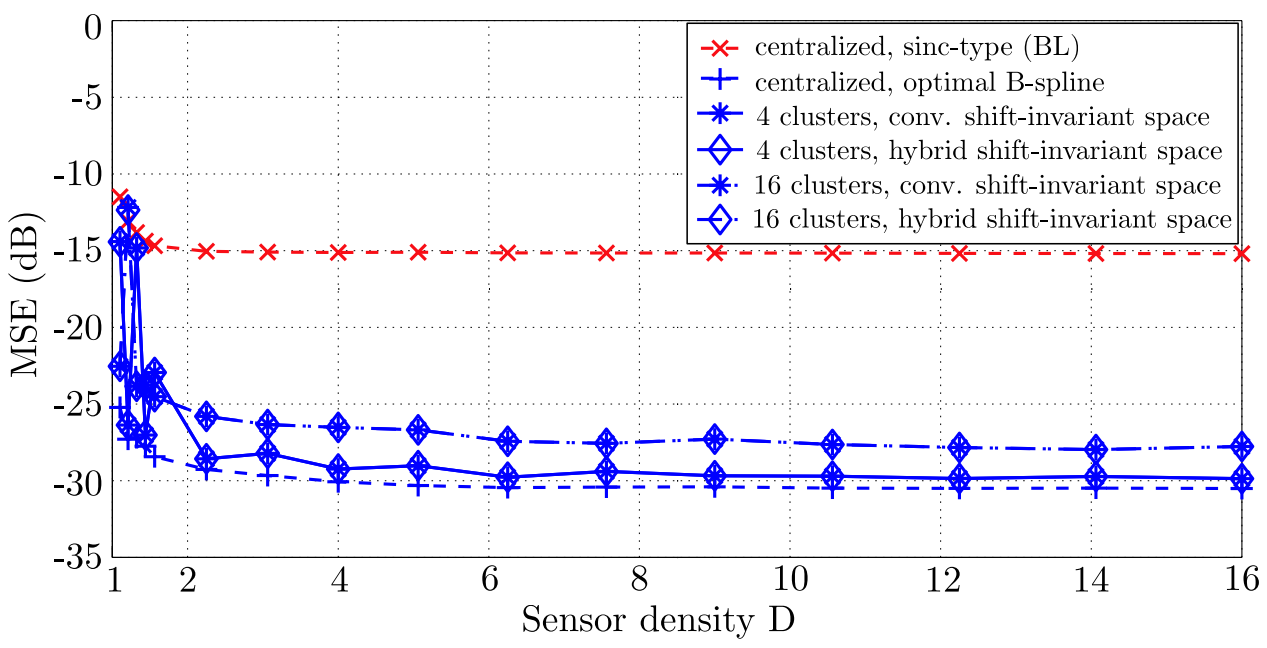

(a)

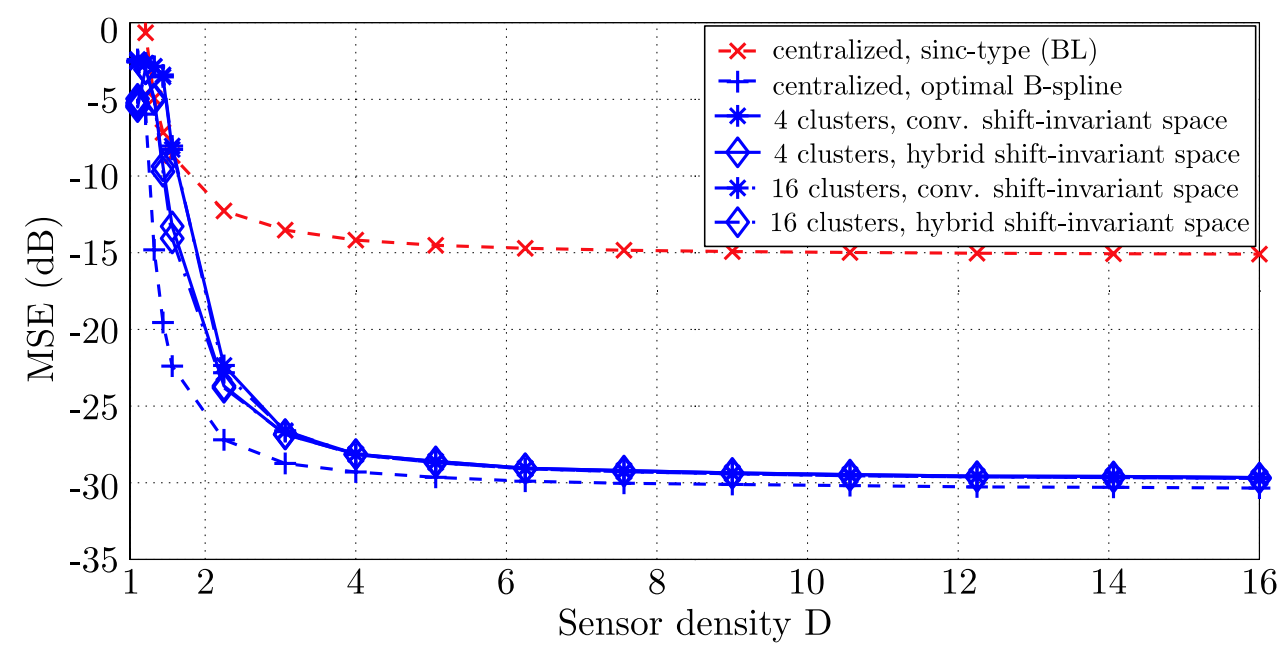

(b)

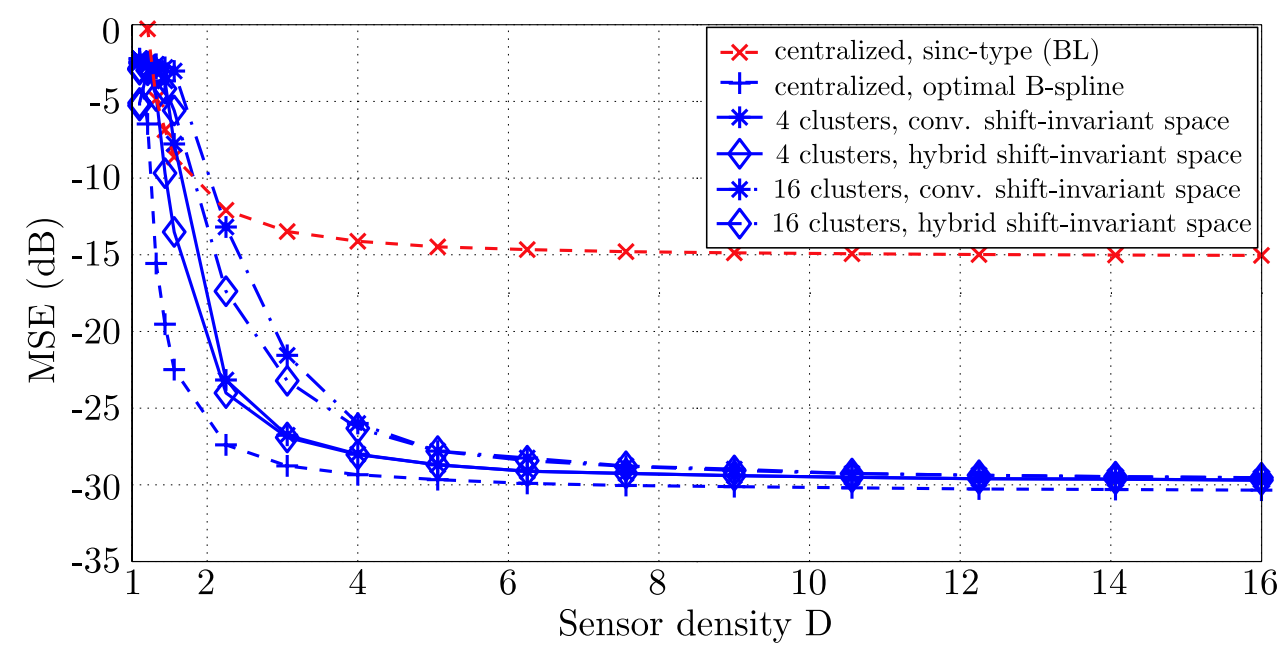

(c)

Figure 3.8: Reconstruction of fields from $V(\bar{g})$ : MSE versus sensor node density achieved with centralized bandlimited reconstruction, centralized B-spline reconstruction, and clustered B-spline reconstruction using 4 and 16 non-overlapping subregions for the case of no measurement noise: (a) sensor node placement on square lattice, (b) square lattice with jitter, (c) random sensor node placement. 


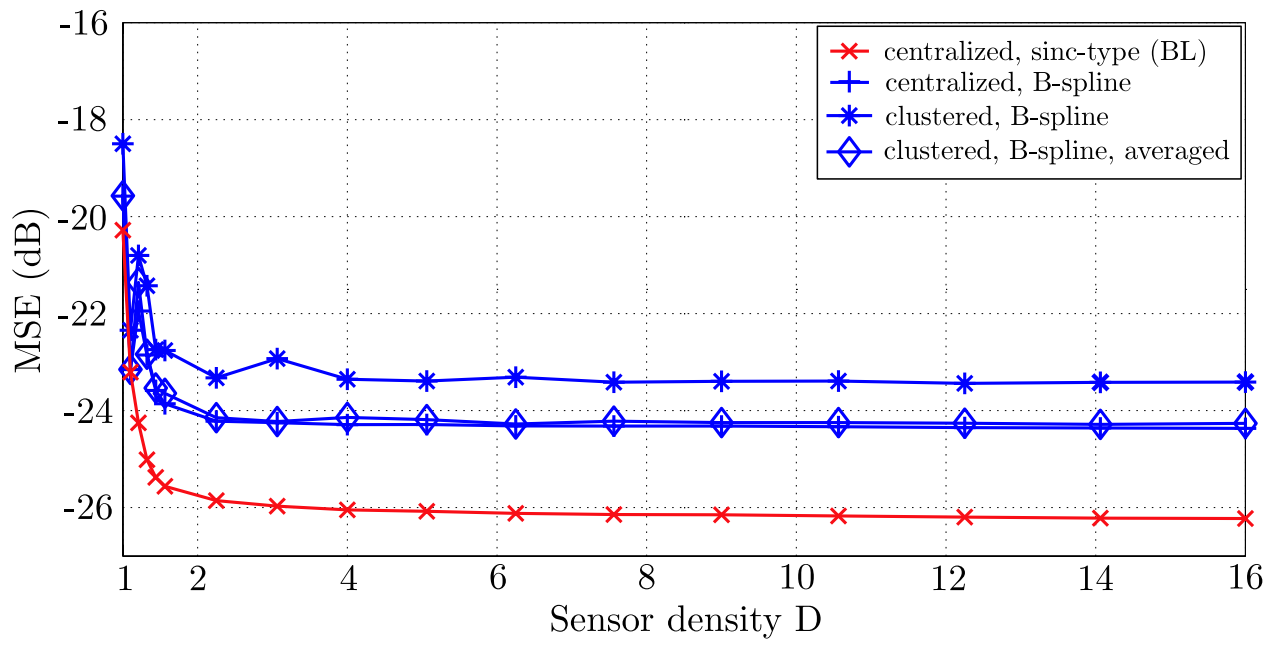

(a)

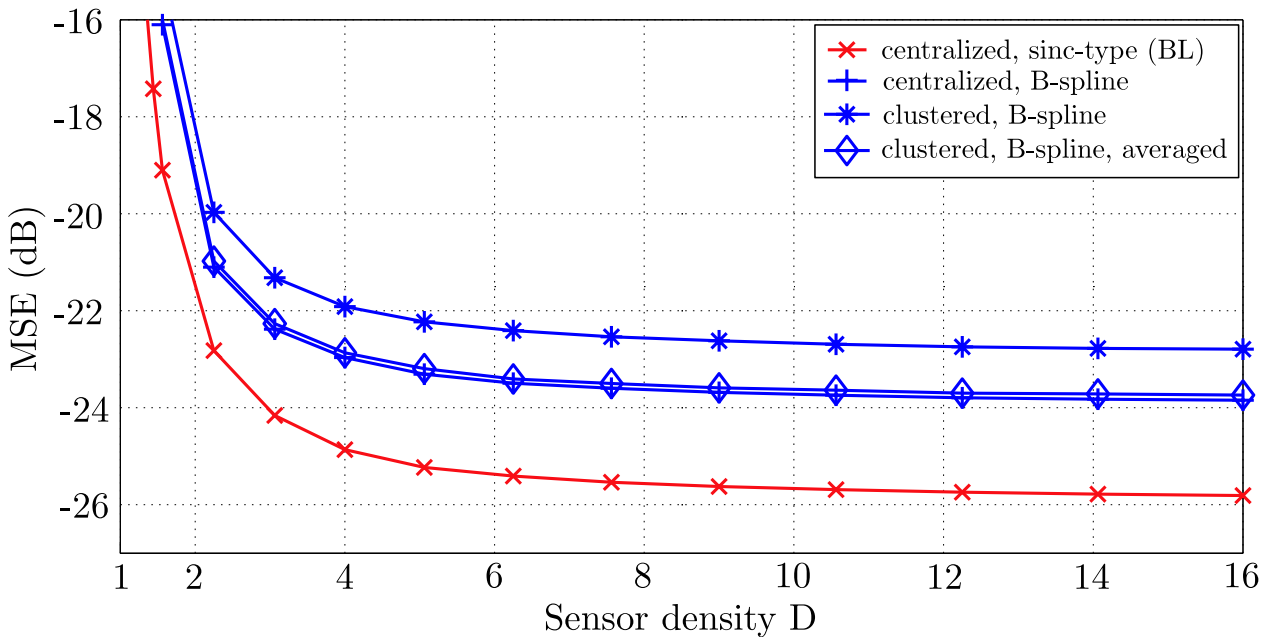

(b)

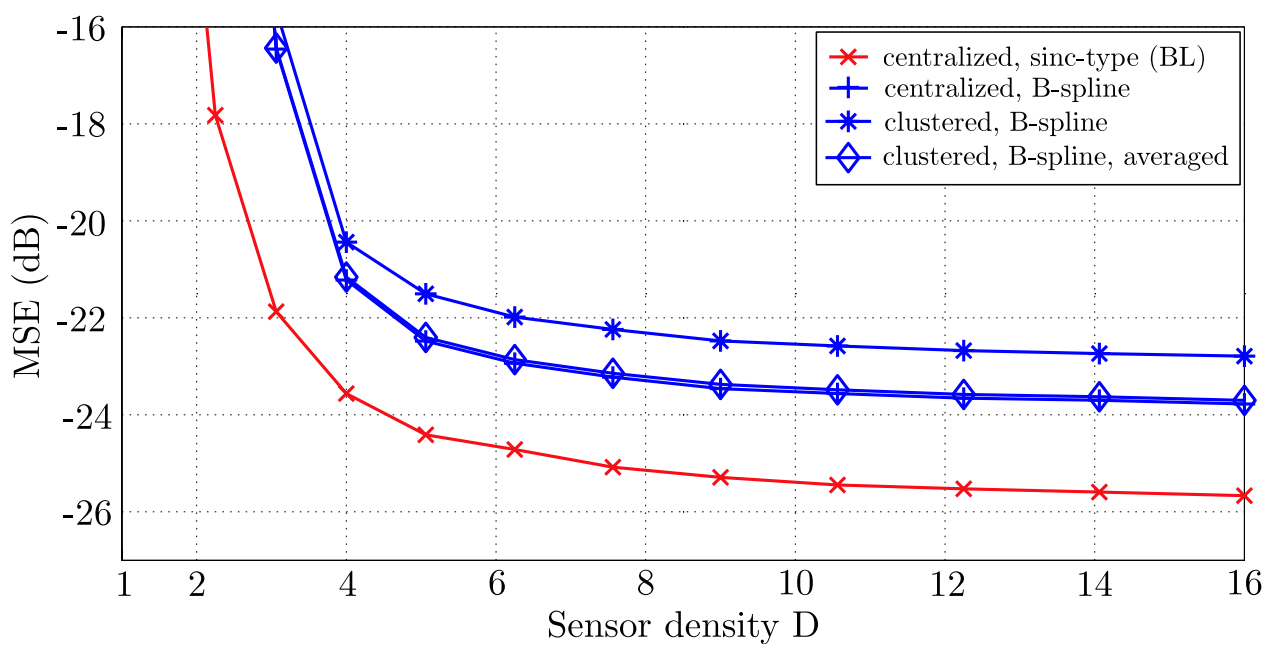

(c)

Figure 3.9: Averaging of coefficient estimates between two clusters for the measurement noise-less case and (a) uniform placement on a square lattice, (b) square lattice with jitter, (c) random placement. 

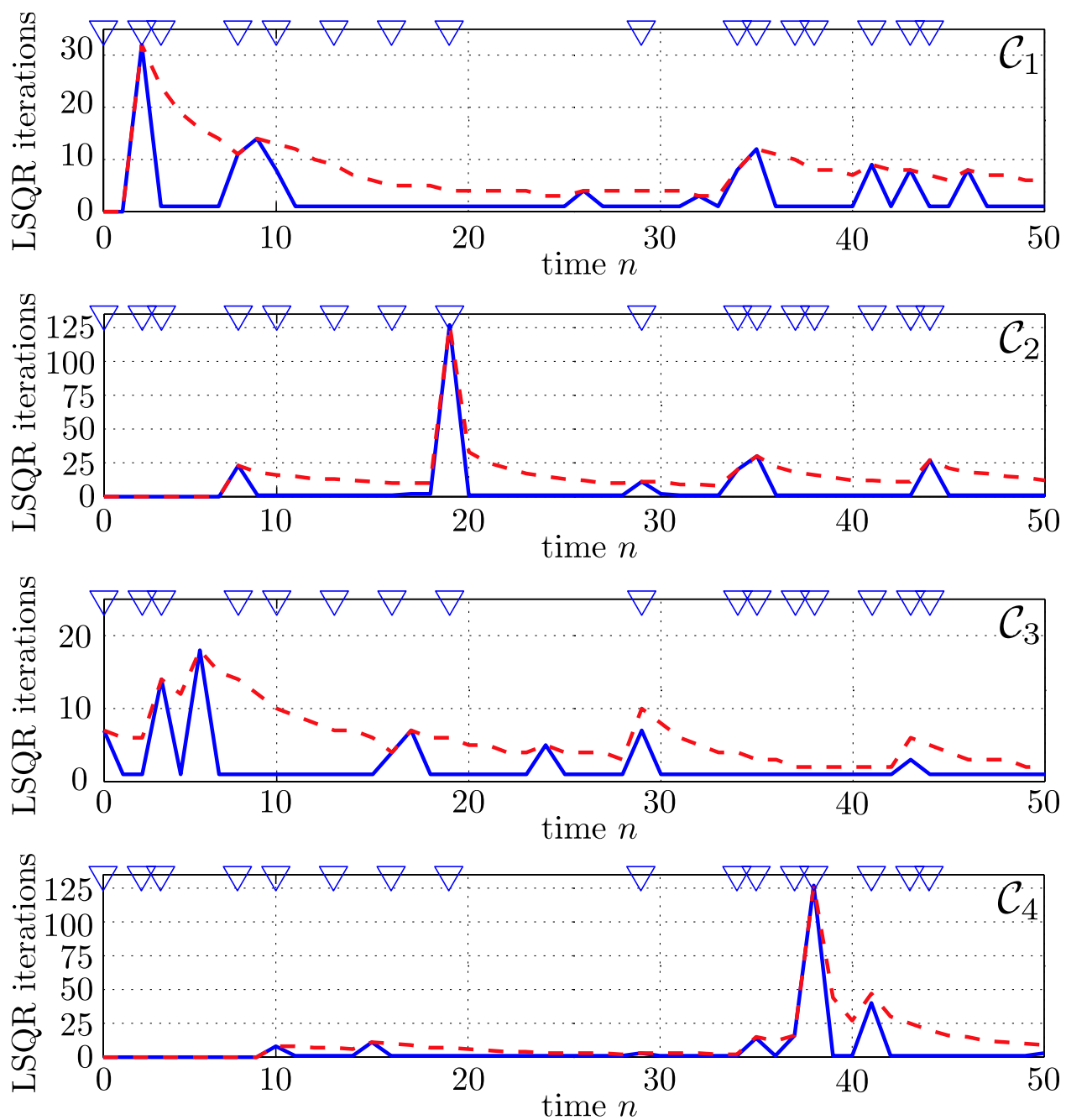

Figure 3.10: Number of LSQR iterations required to obtain a normalized square error of $-10 \mathrm{~dB}$ versus time $n$ for each of the four clusters. Solid blue lines correspond to proposed LSQR initialization and dashed red lines correspond to standard LSQR initialization. The activation times of the sources are marked with $\nabla$ at the top of each plot.

area and achieves the quality of unclustered reconstruction. Averaging of coefficients is therefore a means to compensate for the penalty introduced by clustering. Note, however, that it is not possible to do coefficient averaging if different spline orders are used in the clusters, i.e., using hybrid shift-invariant spaces in combination with averaging is not possible in general.

\subsubsection{Reconstruction of Time-varying Fields}

We illustrate the performance of the reconstruction using the iterative LSQR algorithm for a time-varying diffusion field. The sensor node measurements were constructed according to (3.19) with $P=15$ sources activated at time instants $t_{p}$ Poisson distributed over the interval $[0,50 T]$. The WSN consisted of $I=30^{2}=900$ sensor nodes deployed regularly on a square grid over the region $\mathcal{A}$. For the reconstruction, we used B-spline 


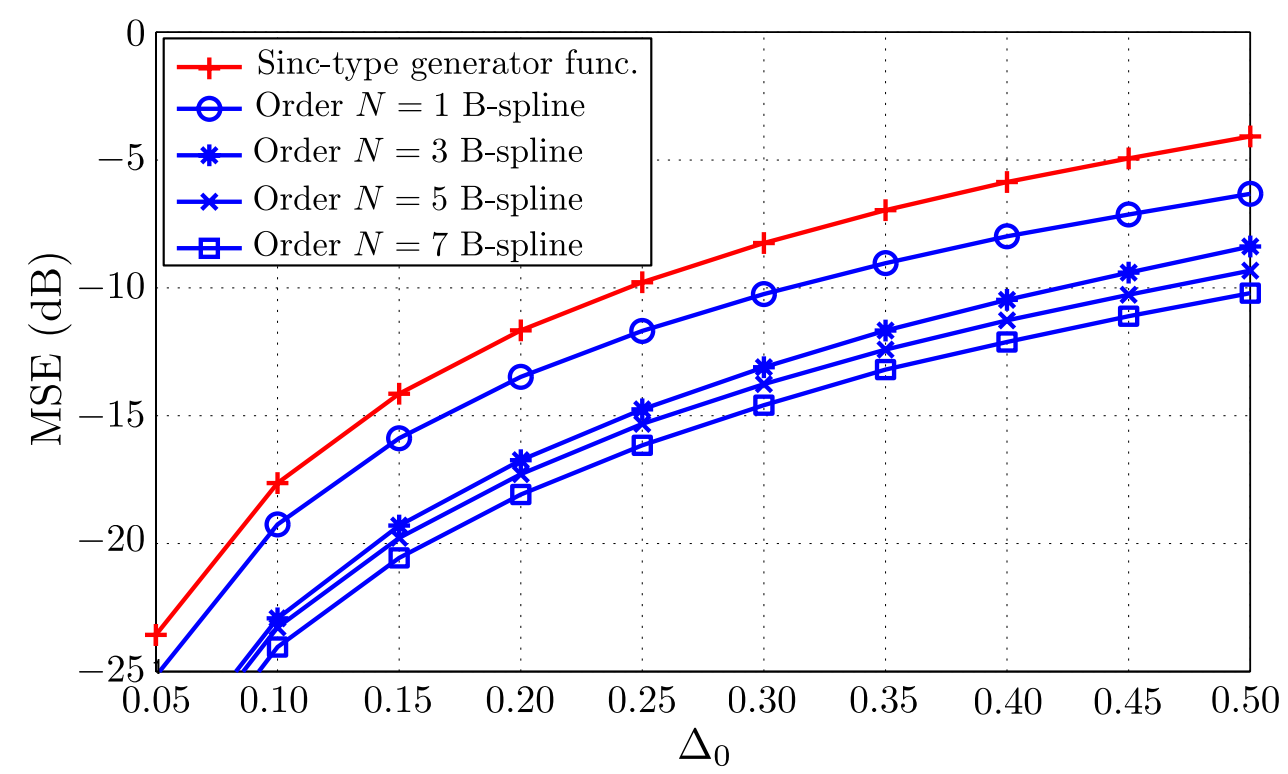

Figure 3.11: Mean square error (MSE) versus maximal localization error $\Delta_{0}$ for bandlimited reconstruction and for field reconstruction using B-spline orders $N \in\{1,3,5,7\}$.

generator functions whose order was empirically optimized within each cluster. Figure 3.10 shows for each of the four clusters the number of LSQR iterations required to obtain a normalized squared error of $-10 \mathrm{~dB}$. We note that before the first source becomes active, the sensor node measurements are all zero and therefore no reconstruction is performed, in which case the number of iterations equals 0 . We compare LSQR with the standard initialization (an all-zero vector) and LSQR initialized using the field coefficients estimated in the previous time-slot. The activation times of the sources are marked with $\nabla$ at the top of each plot. It can be seen that, as expected, the proposed initialization indeed leads to a significantly smaller number of iterations than with the standard initialization. Furthermore, the number of LSQR iterations is quite small most of the time. Only if a source becomes active in a given cluster so that the field changes noticeable, the corresponding cluster head needs to perform a larger number of iterations. Note that source activation mostly affects only a single cluster, e.g., at time $n=19$ a source becomes active in cluster $\mathcal{C}_{2}$ and only the corresponding cluster head has increased workload whereas the other cluster heads need to perform only very few updates at this time instant to track the diffusion process.

\subsubsection{Influence of Sensor Node Localization Error}

We compare the impact of uniformly distributed localization errors on the reconstruction quality for different spline orders and for bandlimited reconstruction. The source field was taken from the shift-invariant space $V\left(b_{N}\right)$ and $V\left(g_{\mathrm{BL}}\right)$ respectively. Field reconstruction was performed using the correct spline order (therefore avoiding a systematic mismatch) but incorrect sensor node positions. Localization errors were uniformly distributed in the interval $\left[-\Delta_{0}, \Delta_{0}\right]$ with $\Delta_{0}$ ranging from 0 to 0.5 . Figure 3.11 shows the MSE obtained for different B-spline orders versus the maximal localization error $\Delta_{0}$. It is seen that all B-spline reconstructions are less sensitive to localization errors than bandlimited reconstruction. In this scenario, B-spline order $N=0$ 
(not shown) is actually completely insensitive against sensor node localization errors (i.e., there is no reconstruction error). For $N>0$, the reconstruction MSE decreases with increasing order, i.e., large spline orders are less sensitive to localization errors. This can be attributed to the fact that the spline curvature about the origin is smaller for larger spline order.

\subsection{Conclusions}

We proposed a cluster-based sensor network architecture for sampling and reconstruction of non-bandlimited fields. Our approach builds on recent progress regarding non-uniform sampling in shift-invariant spaces and allows excellent reconstruction quality while requiring low communication overhead and low computational complexity that is linear in the number of sensor nodes. We showed that a placement of the sensor nodes on a regular grid is preferable to jittered and random placement, but deploying more sensor nodes, i.e., significant oversampling, can make up for the difference and allows the same reconstruction quality. We introduced the concept of hybrid shift-invariant spaces which allow to better adapt our model to the source field and attain better reconstruction quality. Furthermore, our scheme allows centralized reconstruction using a fusion center as well as clustered-based and diffusion-based reconstruction. In the latter two cases the reconstruction problem is partitioned and distributed over the sensor network, thereby reducing the communication overhead and computational complexity. We showed that the penalty introduced by clustering can be tackled by averaging the estimation results between overlapping clusters, which allows to attain the reconstruction quality of the unclustered, centralized scheme. Complexity of the reconstruction of time-varying fields can be reduced significantly by initializing an iterative solver with the field coefficients estimated in the previous time-slot. We analytically and numerically investigated the sensitivity of our scheme to sensor node localization errors and showed that it is less sensitive than bandlimited reconstruction, irrespective of the spline order used. 


\section{A Proofs}

In the appendix we outline the proof of Theorem 1. The proof is based on the corresponding 1-D result in [51]. For the 1-D case, we sort the sensor node positions in increasing order $x_{j}<x_{j+1}$ and assume that there is a lower bound on their distance, $x_{j+1}-x_{j} \geq s>0$. If the maximum gap $\sup _{j \in \mathbb{Z}}\left(x_{j+1}-x_{j}\right)=\delta_{x}<1$ is strictly smaller than 1 , then there exist constants $A_{1}, A_{2}>0$ depending only on the maximum gap $\delta_{x}$ and the separation constant $s$, such that

$$
A_{1}\|f\|_{2}^{2} \leq \sum_{j \in \mathbb{Z}}\left|f\left(x_{j}\right)\right|^{2} \leq A_{2}\|f\|_{2}^{2} \quad \text { for all } f \in V\left(\tilde{b}_{N}\right)
$$

In the 2-D case, every $f \in V\left(b_{N}\right)$ can be written as

$$
f(x, y)=\sum_{k, l \in \mathbb{Z}} c_{k, l} \tilde{b}_{N}(x-k) \tilde{b}_{N}(y-l)
$$

for some coefficients $c_{k, l}$. For fixed $x$, the function $y \rightarrow f(x, y)$ is

$$
f(x, y)=\sum_{l \in \mathbb{Z}}\left(\sum_{k \in \mathbb{Z}} c_{k, l} \tilde{b}_{N}(x-k)\right) \tilde{b}_{N}(y-l),
$$

is in the shift-invariant space $V\left(\tilde{b}_{N}\right)$ with respect to the variable $y$. One checks that the coefficients $a_{l}=$ $\sum_{k \in \mathbb{Z}} c_{k, l} \tilde{b}_{N}(x-k)$ are indeed in $\ell^{2}(\mathbb{Z})$. Similarly, for fixed $y$, the function $x \rightarrow f(x, y)$ is a function in the shift-invariant space $V\left(\tilde{b}_{N}\right)$ of the variable $x$. Since the 1-D estimates of equation (3.22) depend only on the maximum gap $\delta_{x}$ (and the separation $s$ ), we obtain for all $j \in \mathbb{Z}$

$$
A_{1}^{2} \int_{-\infty}^{\infty}\left|f\left(x_{j}, y\right)\right|^{2} d y \leq \sum_{k \in \mathbb{Z}}\left|f\left(x_{j}, y_{j k}\right)\right|^{2} \leq A_{2}^{2} \int_{-\infty}^{\infty}\left|f\left(x_{j}, y\right)\right|^{2} d y
$$

Equation (3.22) yields for all $y$

$$
A_{1}^{2} \int_{\infty}^{\infty}|f(x, y)|^{2} d x \leq \sum_{j \in \mathbb{Z}}\left|f\left(x_{j}, y\right)\right|^{2} \leq A_{2}^{2} \int_{-\infty}^{\infty}|f(x, y)|^{2} d x
$$

With a combination of these two estimates we arrive at

$$
\begin{aligned}
& A_{1}^{4} \int_{-\infty}^{\infty} \int_{-\infty}^{\infty}|f(x, y)|^{2} d x d y \\
& \leq A_{1}^{2} \int_{-\infty}^{\infty}\left(\sum_{j \in \mathbb{Z}}\left|f\left(x_{j}, y\right)\right|^{2}\right) d y \\
& \leq \sum_{j, k \in \mathbb{Z}}\left|f\left(x_{j}, y_{j k}\right)\right|^{2} \\
& \leq A_{2}^{4} \int_{-\infty}^{\infty}|f(x, y)|^{2} .
\end{aligned}
$$




\section{B Calculation of Basis-Splines}

We present two equivalent (analytical) methods to calculate B-splines. Here, we only consider 1-D B-splines $\tilde{b}_{N}(x)$; their 2-D equivalents $b_{N}(x, y)$ can be constructed according to (3.2).

\section{B.1 Calculation Using Convolution}

We start with the B-spline of order $N=0$, which equals the rectangular function, i.e.,

$$
\tilde{b}_{0}(x)=\Pi(x) \triangleq \begin{cases}1, & -\frac{1}{2} \leq x<\frac{1}{2} \\ 0, & \text { else }\end{cases}
$$

The higher order B-splines are obtained by $N$-fold convolution of the rectangular function. We get

$$
\begin{aligned}
& \tilde{b}_{1}(x)=\tilde{b}_{0}(x) * \Pi(x)=\int_{-\infty}^{\infty} \tilde{b}_{0}\left(x^{\prime}\right) \tilde{b}_{0}\left(x-x^{\prime}\right) d x^{\prime}=\left\{\begin{array}{cc}
\int_{-1 / 2}^{x+1 / 2} 1 d x^{\prime}=\left.x^{\prime}\right|_{-1 / 2} ^{x+1 / 2}=x+1, & -1 \leq x \leq 0, \\
\int_{x-1 / 2}^{1 / 2} 1 d x^{\prime}=\left.x^{\prime}\right|_{x-1 / 2} ^{1 / 2}=1-x, & 0 \leq x \leq 1, \\
0, & \text { else },
\end{array}\right\} \\
& =1-|x| \text { for }-1 \leq x \leq 1 \text {; } \\
& \tilde{b}_{2}(x)=\tilde{b}_{1}(x) * \Pi(x)= \begin{cases}\frac{x^{2}}{2}+\frac{3 x}{2}+\frac{9}{8}, & -3 / 2 \leq x<-1 / 2, \\
-x^{2}+\frac{3}{4}, & -1 / 2 \leq x<1 / 2, \\
\frac{x^{2}}{2}-\frac{3 x}{2}+\frac{9}{8}, & 1 / 2 \leq x \leq 3 / 2, \\
0, & \text { else }\end{cases} \\
& \tilde{b}_{3}(x)=\tilde{b}_{2}(x) * \Pi(x)= \begin{cases}\frac{x^{3}}{6}+x^{2}+2 x+\frac{4}{3}, & -2 \leq x<-1, \\
-\frac{x^{3}}{2}-x^{2}+\frac{2}{3}, & -1 \leq x<0, \\
\frac{x^{3}}{2}-x^{2}+\frac{2}{3}, & 0 \leq x<1, \\
-\frac{x^{3}}{6}+x^{2}-2 x+\frac{4}{3}, & 1 \leq x \leq 2, \\
0, & \text { else }\end{cases} \\
& \tilde{b}_{4}(x)=\tilde{b}_{3}(x) * \Pi(x)= \begin{cases}\frac{x^{4}}{24}+\frac{5 x^{3}}{12}+\frac{25 x^{2}}{16}+\frac{125 x}{48}+\frac{625}{384}, & -5 / 2 \leq x<-3 / 2, \\
-\frac{x^{4}}{6}-\frac{5 x^{3}}{6}-\frac{5 x^{2}}{4}-\frac{5 x}{24}+\frac{55}{96}, & -3 / 2 \leq x<-1 / 2, \\
\frac{x^{4}}{4}-\frac{5 x^{2}}{8}+\frac{115}{192}, & -1 / 2 \leq x<1 / 2, \\
-\frac{x^{4}}{6}+\frac{5 x^{3}}{6}-\frac{5 x^{2}}{4}+\frac{5 x}{24}+\frac{55}{96}, & 1 / 2 \leq x<3 / 2, \\
\frac{x^{4}}{24}-\frac{5 x^{3}}{12}+\frac{25 x^{2}}{16}-\frac{125 x}{48}+\frac{625}{384}, & 3 / 2 \leq x \leq 5 / 2, \\
0, & \text { else. }\end{cases}
\end{aligned}
$$

Further convolution with the rectangular function leads to B-splines of higher orders. 


\section{B.2 Cox-de Boor Algorithm}

An alternative for the calculation of B-splines is the Cox-de Boor algorithm, which we will use to obtain the B-splines of the next higher orders. The algorithm is based on an ordered set of numbers $\mathcal{X}=\left\{x_{0}, \ldots, x_{M}\right\}$, called knots, where $x_{0} \leq x_{1} \leq \ldots \leq x_{M}$.

The B-splines consist of piecewise polynomials, where the $m$ th polynomial of the order- $N$ B-spline can recursively be calculated by the Cox-de Boor recursion formula [54] as

$$
P_{m, N}(x)=\frac{x-x_{i}}{x_{i+N}-x_{i}} P_{i, N-1}(x)+\frac{x_{i+N+1}-x}{x_{i+N+1}-x_{i+1}} P_{i+1, N-1}(x)
$$

with

$$
P_{m, 0}(x)= \begin{cases}1, & x_{i} \leq x<x_{i+1} \\ 0, & \text { else }\end{cases}
$$

The B-splines used in this dissertation are obtained by the Cox-de Boor recursion formula for an integer knot sequence, i.e., $x_{m} \in \mathbb{Z}$, followed by appropriately shifting the resulting polynomials to get zero-centered B-splines. The results for the orders $N=1, \ldots, 4$ equal those presented in Section 3.B.1, which is why we continue to list the results for the remaining orders $N=5, \ldots, 8$ used in this dissertation:

$$
\begin{aligned}
& \tilde{b}_{5}(x)= \begin{cases}\frac{x^{5}}{120}+\frac{x^{4}}{8}+\frac{3 x^{3}}{4}+\frac{9 x^{2}}{4}+\frac{27 x}{8}+\frac{81}{40}, & -3 \leq x \leq 2, \\
-\frac{x^{5}}{24}-\frac{3 x^{4}}{8}-\frac{5 x^{3}}{4}-\frac{7 x^{2}}{4}-\frac{5 x}{8}+\frac{17}{40}, & -2 \leq x \leq-1, \\
\frac{x^{5}}{12}+\frac{x^{4}}{4}-\frac{x^{2}}{2}+\frac{11}{20}, & -1 \leq x \leq 0, \\
-\frac{x^{5}}{12}+\frac{x^{4}}{4}-\frac{x^{2}}{2}+\frac{11}{20}, & 0 \leq x \leq 1, \\
\frac{x^{5}}{24}-\frac{3 x^{4}}{8}+\frac{5 x^{3}}{4}-\frac{7 x^{2}}{4}+\frac{5 x}{8}+\frac{17}{40}, & 1 \leq x \leq 2, \\
-\frac{x^{5}}{120}+\frac{x^{4}}{8}-\frac{3 x^{3}}{4}+\frac{9 x^{2}}{4}-\frac{27 x}{8}+\frac{81}{40}, & 2 \leq x \leq 3, \\
0, & \text { else }\end{cases} \\
& \tilde{b}_{6}(x)= \begin{cases}\frac{x^{6}}{720}+\frac{7 x^{5}}{240}+\frac{49 x^{4}}{192}+\frac{343 x^{3}}{288}+\frac{2401 x^{2}}{768}+\frac{16807 x}{3840}+\frac{117649}{46080}, & -7 / 2 \leq x<-5 / 2, \\
-\frac{x^{6}}{120}-\frac{7 x^{5}}{60}-\frac{21 x^{4}}{32}-\frac{133 x^{3}}{72}-\frac{329 x^{2}}{128}-\frac{1267 x}{960}+\frac{1379}{7680}, & -5 / 2 \leq x<-3 / 2, \\
\frac{x^{6}}{48}+\frac{7 x^{5}}{48}+\frac{21 x^{4}}{64}+\frac{35 x^{3}}{288}-\frac{91 x^{2}}{256}+\frac{7 x}{768}+\frac{7861}{15360}, & -3 / 2 \leq x \leq-1 / 2, \\
-\frac{x^{6}}{36}+\frac{7 x^{4}}{48}-\frac{77 x^{2}}{192}+\frac{5887}{11520}, & -1 / 2 \leq x \leq 1 / 2, \\
\frac{x^{6}}{48}-\frac{7 x^{5}}{48}+\frac{21 x^{4}}{64}-\frac{35 x^{3}}{288}-\frac{91 x^{2}}{256}-\frac{7 x}{768}+\frac{7861}{15360}, & 1 / 2 \leq x \leq 3 / 2, \\
-\frac{x^{6}}{120}+\frac{7 x^{5}}{60}-\frac{21 x^{4}}{32}+\frac{133 x^{3}}{72}-\frac{329 x^{2}}{128}+\frac{1267 x}{960}+\frac{1379}{7680}, & 3 / 2 \leq x \leq 5 / 2, \\
\frac{x^{6}}{720}-\frac{7 x^{5}}{240}+\frac{49 x^{4}}{192}-\frac{343 x^{3}}{288}+\frac{2401 x^{2}}{768}-\frac{16807 x}{3840}+\frac{117649}{46080}, & 5 / 2 \leq x \leq 7 / 2, \\
0, & \text { else; }\end{cases}
\end{aligned}
$$




$$
\begin{aligned}
& \tilde{b}_{7}(x)= \begin{cases}\frac{x^{7}}{5040}+\frac{x^{6}}{180}+\frac{x^{5}}{15}+\frac{4 x^{4}}{9}+\frac{16 x^{3}}{9}+\frac{64 x^{2}}{15}+\frac{256 x}{45}+\frac{1024}{315}, & -4 \leq x<-3, \\
-\frac{x^{7}}{720}-\frac{x^{6}}{36}-\frac{7 x^{5}}{30}-\frac{19 x^{4}}{18}-\frac{49 x^{3}}{18}-\frac{23 x^{2}}{6}-\frac{217 x}{90}-\frac{139}{630}, & -3 \leq x<-2, \\
\frac{x^{7}}{240}+\frac{x^{6}}{20}+\frac{7 x^{5}}{30}+\frac{x^{4}}{2}+\frac{7 x^{3}}{18}-\frac{x^{2}}{10}+\frac{7 x}{90}+\frac{103}{210}, & -2 \leq x<-1, \\
-\frac{x^{7}}{144}-\frac{x^{6}}{36}+\frac{x^{4}}{9}-\frac{x^{2}}{3}+\frac{151}{315}, & -1 \leq x<0, \\
\frac{x^{7}}{144}-\frac{x^{6}}{36}+\frac{x^{4}}{9}-\frac{x^{2}}{3}+\frac{151}{315}, & 0 \leq x<1, \\
-\frac{x^{7}}{240}+\frac{x^{6}}{20}-\frac{7 x^{5}}{30}+\frac{x^{4}}{2}-\frac{7 x^{3}}{18}-\frac{x^{2}}{10}-\frac{7 x}{90}+\frac{103}{210}, & 1 \leq x<2, \\
\frac{x^{7}}{720}-\frac{x^{6}}{36}+\frac{7 x^{5}}{30}-\frac{19 x^{4}}{18}+\frac{49 x^{3}}{18}-\frac{23 x^{2}}{6}+\frac{217 x}{90}-\frac{139}{630}, & 2 \leq x<3, \\
-\frac{x^{7}}{5040}+\frac{x^{6}}{180}-\frac{x^{5}}{15}+\frac{4 x^{4}}{9}-\frac{16 x^{3}}{9}+\frac{64 x^{2}}{15}-\frac{256 x}{45}+\frac{1024}{315}, & 3 \leq x<4, \\
0, & \text { else; }\end{cases} \\
& \tilde{b}_{8}(x)= \begin{cases}\frac{x^{8}}{40320}+\frac{x^{7}}{1120}+\frac{9 x^{6}}{640}+\frac{81 x^{5}}{640}+\frac{729 x^{4}}{1024}+\frac{6561 x^{3}}{2560}+\frac{59049 x^{2}}{10240}+\frac{531441 x}{71680}+\frac{4782969}{1146880}, & -9 / 2 \leq x<-7 / 2, \\
-\frac{x^{8}}{5040}-\frac{3 x^{7}}{560}-\frac{x^{6}}{16}-\frac{131 x^{5}}{320}-\frac{209 x^{4}}{128}-\frac{5123 x^{3}}{1280}-\frac{1465 x^{2}}{256}-\frac{146051 x}{35840}-\frac{122729}{143360}, & -7 / 2 \leq x<-5 / 2, \\
\frac{x^{8}}{1440}+\frac{x^{7}}{80}+\frac{3 x^{6}}{32}+\frac{119 x^{5}}{320}+\frac{207 x^{4}}{256}+\frac{1127 x^{3}}{1280}+\frac{195 x^{2}}{512}+\frac{1457 x}{5120}+\frac{145167}{286720}, & -5 / 2 \leq x<-3 / 2, \\
-\frac{x^{8}}{720}-\frac{x^{7}}{80}-\frac{3 x^{6}}{80}-\frac{7 x^{5}}{320}+\frac{9 x^{4}}{128}-\frac{7 x^{3}}{1280}-\frac{363 x^{2}}{1280}-\frac{x}{5120}+\frac{64929}{143360}, & -3 / 2 \leq x<-1 / 2, \\
\frac{x^{8}}{576}-\frac{x^{6}}{64}+\frac{43 x^{4}}{512}-\frac{289 x^{2}}{1024}+\frac{259723}{573440}, & -1 / 2 \leq x<1 / 2, \\
-\frac{x^{8}}{720}+\frac{x^{7}}{80}-\frac{3 x^{6}}{80}+\frac{7 x^{5}}{320}+\frac{9 x^{4}}{128}+\frac{7 x^{3}}{1280}-\frac{363 x^{2}}{1280}+\frac{x}{5120}+\frac{64929}{143360}, & 1 / 2 \leq x<3 / 2, \\
\frac{x^{8}}{1440}-\frac{x^{7}}{80}+\frac{3 x^{6}}{32}-\frac{119 x^{5}}{320}+\frac{207 x^{4}}{256}-\frac{1127 x^{3}}{1280}+\frac{195 x^{2}}{512}-\frac{1457 x}{5120}+\frac{145167}{286720}, & 3 / 2 \leq x<5 / 2, \\
-\frac{x^{8}}{5040}+\frac{3 x^{7}}{560}-\frac{x^{6}}{16}+\frac{131 x^{5}}{320}-\frac{209 x^{4}}{128}+\frac{5123 x^{3}}{1280}-\frac{1465 x^{2}}{256}+\frac{146051 x}{35840}-\frac{122729}{143360}, & 5 / 2 \leq x<7 / 2, \\
\frac{x^{8}}{40320}-\frac{x^{7}}{1120}+\frac{9 x^{6}}{640}-\frac{81 x^{5}}{640}+\frac{729 x^{4}}{1024}-\frac{6561 x^{3}}{2560}+\frac{59049 x^{2}}{10240}-\frac{531441 x}{71680}+\frac{4782969}{1146880}, & 7 / 2 \leq x<9 / 2, \\
0, & \text { else. }\end{cases}
\end{aligned}
$$




\section{C Example Calculation of $\mathrm{G}_{\mathrm{g}}$}

We calculate the elements of the Gramian $\mathbf{G}_{\mathbf{g}}=\iint_{\mathcal{A}} \mathbf{g}(x, y) \mathbf{g}^{T}(x, y) d x d y$ of the vector $[\mathbf{g}]_{p}(x, y)=b_{N}(x-$ $\left.k_{p}, y-l_{p}\right)$ with $k_{p}=k_{0}+((p-1) \bmod K)$, and $l_{p}=l_{0}+\left\lfloor\frac{p-1}{K}\right\rfloor$.

$$
\begin{aligned}
{\left[\mathbf{G}_{\mathbf{g}}\right]_{p, p^{\prime}} } & =\iint_{\mathcal{A}} b_{N}\left(x-k_{p}, y-l_{p}\right) b_{N}\left(x-k_{p^{\prime}}, y-l_{p^{\prime}}\right) d x d y \\
& =\iint_{\mathcal{A}} \tilde{b}_{N}\left(x-k_{p}\right) \tilde{b}_{N}\left(y-l_{p}\right) \tilde{b}_{N}\left(x-k_{p^{\prime}}\right) \tilde{b}_{N}\left(y-l_{p^{\prime}}\right) d x d y \\
& =\int_{\mathcal{A}_{x}} \tilde{b}_{N}\left(x-k_{p}\right) \tilde{b}_{N}\left(x-k_{p^{\prime}}\right) d x \int_{\mathcal{A}_{y}} \tilde{b}_{N}\left(y-l_{p}\right) \tilde{b}_{N}\left(y-l_{p^{\prime}}\right) d y .
\end{aligned}
$$

The matrix $\mathbf{G}_{\mathbf{g}}$ is symmetric and has block-Toeplitz structure

$$
\mathbf{G}_{\mathbf{g}}=\left(\begin{array}{ccccc}
\mathbf{B}_{0} & \mathbf{B}_{1}^{T} & \mathbf{B}_{2}^{T} & \ldots & \mathbf{B}_{L}^{T} \\
\mathbf{B}_{1} & \mathbf{B}_{0} & \mathbf{B}_{1}^{T} & \ldots & \mathbf{B}_{L-1}^{T} \\
\mathbf{B}_{2} & \mathbf{B}_{1} & \mathbf{B}_{0} & \ldots & \mathbf{B}_{L-2}^{T} \\
\vdots & \vdots & \vdots & \ddots & \vdots \\
\mathbf{B}_{L-1} & \mathbf{B}_{L-2} & \mathbf{B}_{L-3} & \ldots & \mathbf{B}_{1}^{T} \\
\mathbf{B}_{L} & \mathbf{B}_{L-1} & \mathbf{B}_{L-2} & \ldots & \mathbf{B}_{0}
\end{array}\right)
$$

with banded blocks $\mathbf{B}_{0}, \ldots, \mathbf{B}_{L} \in \mathbb{R}^{K \times K}$ that have Toeplitz structure as well. For $N=1$ we get

$$
\begin{aligned}
{\left[\mathbf{B}_{0}\right]_{p, p^{\prime}} } & =\int_{\mathcal{A}_{x}} \tilde{b}_{1}\left(x-k_{p}\right) \tilde{b}_{1}\left(x-k_{p^{\prime}}\right) d x \int_{\mathcal{A}_{y}}\left(\tilde{b}_{1}\left(y-l_{p}\right)\right)^{2} d y \\
& = \begin{cases}\frac{4}{9}, & \text { if }\left|p-p^{\prime}\right| \leq 1, \\
0, & \text { else },\end{cases} \\
{\left[\mathbf{B}_{1}\right]_{p, p^{\prime}} } & =\int_{\mathcal{A}_{x}} \tilde{b}_{1}\left(x-k_{p}\right) \tilde{b}_{1}\left(x-k_{p^{\prime}}\right) d x \int_{\mathcal{A}_{y}} \tilde{b}_{1}\left(y-l_{p}\right) \tilde{b}_{1}\left(y-l_{p}-1\right) d y \\
& = \begin{cases}\frac{4}{9}, & \text { if }\left|p-p^{\prime}\right| \leq 1, \\
0, & \text { else },\end{cases} \\
{\left[\mathbf{B}_{2}\right]_{p, p^{\prime}} } & =\ldots=\left[\mathbf{B}_{L}\right]_{p, p^{\prime}}=0,
\end{aligned}
$$

where the detailed calculation for each case can be found below. Note that although the matrices $\mathbf{B}_{l}$ are equal for $N=1$, this is not the case in general.

For $p=p^{\prime}$ we have:

$$
\begin{aligned}
{\left[\mathbf{B}_{0}\right]_{p, p} } & =\int_{k_{p}-1}^{k_{p}+1} \tilde{b}_{1}^{2}\left(x-k_{p}\right) d x \int_{l_{p}-1}^{l_{p}+1} \tilde{b}_{1}^{2}\left(y-l_{p}\right) d y \\
& =\int_{-1}^{+1} \tilde{b}_{1}^{2}(x) d x \int_{-1}^{+1} \tilde{b}_{1}^{2}(y) d y=\int_{-1}^{+1}(1-|x|)^{2} d x \int_{-1}^{+1}(1-|y|)^{2} d y \\
& =\left(\int_{-1}^{0}(1+x)^{2} d x+\int_{0}^{1}(1-x)^{2} d x\right)\left(\int_{-1}^{0}(1+y)^{2} d y+\int_{0}^{1}(1-y)^{2} d y\right)
\end{aligned}
$$




$$
\begin{gathered}
=\left(\int_{-1}^{0}\left(x^{2}+2 x+1\right) d x+\int_{0}^{1}\left(x^{2}-2 x+1\right) d x\right)^{2} \\
=\left(\left.\left(\frac{x^{3}}{3}+x^{2}+x\right)\right|_{-1} ^{0}+\left.\left(\frac{x^{3}}{3}-x^{2}+x\right)\right|_{0} ^{1}\right)^{2}=\left(\frac{1}{3}+\frac{1}{3}\right)^{2}=\frac{4}{9} \\
{\left[\mathbf{B}_{1}\right]_{p, p}=\int_{k_{p}-1}^{k_{p}+1} \tilde{b}_{1}^{2}\left(x-k_{p}\right) d x \int_{l_{p}-1}^{l_{p}+2} \tilde{b}_{1}\left(y-l_{p}\right) \tilde{b}_{1}\left(y-l_{p}-1\right) d y} \\
=\frac{2}{3} \int_{-1}^{+2} \tilde{b}_{1}(y) \tilde{b}_{1}(y-1) d y=\frac{2}{3} \int_{0}^{+1}(1-y)(1+y) d x \\
=\frac{2}{3} \int_{0}^{+1}\left(1-y^{2}\right) d x=\left.\frac{2}{3}\left(y-\frac{y^{3}}{3}\right)\right|_{0} ^{1}=\frac{4}{9}
\end{gathered}
$$

For $\left|p-p^{\prime}\right|=1$ we have:

$$
\begin{gathered}
{\left[\mathbf{B}_{0}\right]_{p, p^{\prime}}=\int_{k_{p}-1}^{k_{p}+2} \tilde{b}_{1}\left(x-k_{p}\right) \tilde{b}_{1}\left(x-k_{p}-1\right) d x \int_{l_{p}-1}^{l_{p}+1} \tilde{b}_{1}\left(y-l_{p}\right) \tilde{b}_{1}\left(y-l_{p}\right) d y=\frac{4}{9}} \\
{\left[\mathbf{B}_{1}\right]_{p, p^{\prime}}=\int_{k_{p}-1}^{k_{p}+2} \tilde{b}_{1}\left(x-k_{p}\right) \tilde{b}_{1}\left(x-k_{p}-1\right) d x \int_{l_{p}-1}^{l_{p}+2} \tilde{b}_{1}\left(y-l_{p}\right) \tilde{b}_{1}\left(y-l_{p}-1\right) d y=\frac{4}{9} .}
\end{gathered}
$$

For $\left|p-p^{\prime}\right| \geq 2$ :

$$
\begin{gathered}
{\left[\mathbf{B}_{0}\right]_{p, p^{\prime}}=\int_{k_{p}-1}^{k_{p}+3} \tilde{b}_{1}\left(x-k_{p}\right) \tilde{b}_{1}\left(x-k_{p}-2\right) d x \int_{l_{p}-1}^{l_{p}+1} \tilde{b}_{1}\left(y-l_{p}\right) \tilde{b}_{1}\left(y-l_{p}\right) d y=0,} \\
{\left[\mathbf{B}_{1}\right]_{p, p^{\prime}}=\int_{k_{p}-1}^{k_{p}+3} \tilde{b}_{1}\left(x-k_{p}\right) \tilde{b}_{1}\left(x-k_{p}-2\right) d x \int_{l_{p}-1}^{l_{p}+2} \tilde{b}_{1}\left(y-l_{p}\right) \tilde{b}_{1}\left(y-l_{p}-1\right) d y=0 .}
\end{gathered}
$$




\section{4}

\section{Optimal Power Allocation}

$\mathrm{O}$ $\mathrm{N}$ the basis of the distributed field reconstruction scheme for Wireless Sensor Networks (WSNs) based on shift-invariant spaces, which we developed in the previous chapter, we introduce optimal power allocation schemes. We start with establishing an Amplify-and-Forward (AF) scheme for the transmission of the sensor node measurements to the fusion center. We then derive the Mean Square Error (MSE) of the reconstructed field as a function of the measurement noise at the sensor nodes, the channel gains between sensor nodes and fusion center, the receiver noise variance, and the sensor node positions. We take a closer look at two optimization scenarios and formulate them as convex optimization problems that can be solved numerically. First, we impose a transmit sum-power constraint and formulate the MSE-optimal power allocation. Second, we impose an upper bound on the MSE of the reconstructed field and formulate the optimal power allocation for minimum transmit sum power. For the case of critical sampling we derive closed-form expressions for the optimal power allocation for both optimization scenarios. For Gaussian channels and Rayleigh fading channels, we compare the performance of the proposed power allocation schemes and of uniform power allocation using numerical simulations. 


\subsection{Background and State of the Art}

We take a closer look at the problem of power allocation for the field reconstruction scheme presented in Chapter 3. We consider a centralized setup in which each sensor node sends its observation to a fusion center where a global estimation for the field is made. With analog observations of the sensor nodes, one can transmit the measurements from the sensor nodes to the fusion center using either analog or digital communication. For analog communication, the sensor node measurement is transmitted using an analog modulation scheme for the signal transmission, which is commonly known as Amplify-and-Forward (AF) approach ${ }^{1}$. For digital communication, the sensor node measurements are quantized and converted to bits, possibly channel coded, and transmitted to the fusion center using digital modulation schemes.

The goal of power allocation is to optimally assign transmit powers to the sensor nodes of the WSN, either in order to minimize the mean square estimation error subject to a transmit sum-power constraint or in order to minimize the transmit power subject to an estimation error target. Such problems have been extensively investigated in the literature for linear scalar models. In [55] it was shown that for a Gaussian source, an uncoded AF transmission over a Gaussian coherent Multiple-Access Channel (MAC) outperforms the best approach using separated source and channel coding (cf. source-channel coding separation theorem [56, Chapter 7]), attaining a larger decay rate of the estimation error and leading to an optimal asymptotic scaling behavior. Asymptotic optimality, however, is achieved only if distributed synchronization of the sensor node signals can be arranged. A more realistic and hence widely used assumption is the case of orthogonal channels from the sensor nodes to the fusion center, e.g., by using Time Division Multiple Access (TDMA), Frequency Division Multiple Access (FDMA), or Code Division Multiple Access (CDMA).

A Decentralized Estimation Scheme (DES) usually requires knowledge of the sensor node measurements' Probability Density Functions (pdfs), which can be difficult to characterize for large-scale networks. In an effort to discard the need of pdfs, [57] proposed a universal DES in which the sensor nodes send discrete messages of different length. The length of messages only depends on the local Signal-to-Noise Ratio (SNR) and hence carries information about the reliability of the measurement. The power scheduling for transmission using uncoded Quadrature Amplitude Modulation (QAM) based on this universal DES was studied in [58]. In $[59,60]$, an uncoded AF scheme for estimation in WSN was employed to transmit the sensor node measurements to the fusion center over orthogonal channels and the optimal power allocation was derived for minimization of the mean square estimation error and the transmit power, respectively. In both cases, the optimal power allocation is similar to water-filling, i.e., sensor nodes with poor channel gains or noisy observations remain inactive to save power. The work of [60] was extended to the case of distributed estimation of a random field in [61]. A suboptimal power allocation scheme for the estimation of a random parameter in the presence of noisy links was proposed in [62].

We investigate the problem of power allocation in a WSN using AF transmission to the fusion center as in [60]. However, rather than a scalar model, we consider the linear matrix-vector model used in Chapter 3 for field reconstruction based on shift-invariant spaces. Different from [60], our model makes the optimal power allocation depend on the sensor node positions. We formulate the optimal (in the mean square estimation error

\footnotetext{
${ }^{1}$ Although $\mathrm{AF}$ is well known in relaying scenarios we want to point out that here we are not using a relaying setup.
} 
sense as well as in the transmit sum-power sense) power allocation as a convex optimization problem that can be solved numerically using standard techniques and derive closed-form solutions for the special case of critical sampling. Finally, we compare the derived power allocation schemes with uniform power allocation in numerical simulations.

\subsection{Signal Model}

For the power allocation we augment our signal model introduced in Chapter 3 with a model for the transmission from the sensor nodes to the fusion center. The WSN consists of $I$ sensor nodes deployed over a given region $\mathcal{A}$ to monitor a Two-Dimensional (2-D) physical field $h(x, y)$. Here, $x$ and $y$ denote the spatial coordinates, the position of sensor node $i$ is given by $\left(x_{i}, y_{i}\right)$ and its measurement reads $h\left(x_{i}, y_{i}\right)+v_{i}$, where $v_{i}$ denotes spatially white measurement noise with variance $\sigma_{v_{i}}^{2}$. We assume that the physical field has mean power $\sigma_{h}^{2}$ and moreover $h(x, y) \in V\left(b_{N}\right)$, i.e.,

$$
h(x, y)=\sum_{(k, l) \in \mathbb{A}} c_{k, l} b_{N}(x-k, y-l),
$$

where $\mathbb{A}=\mathbb{Z}^{2} \cap(\mathcal{A}+\mathcal{S})$. As before, reconstruction of $h(x, y)$ from the measurements thus amounts to determining the coefficients $c_{k, l}$.

We adopt an Amplify-and-Forward (AF) transmission strategy $[59,63]$ were each sensor node transmits the scaled measurement $\sqrt{p_{i}}\left(h\left(x_{i}, y_{i}\right)+v_{i}\right)$ to the fusion center. The average transmit power of sensor node $i$ thus equals

$$
P_{i}=p_{i}\left(\sigma_{h}^{2}+\sigma_{v_{i}}^{2}\right)
$$

The transmit sum power is

$$
\sum_{i=1}^{I} P_{i}=\sum_{i=1}^{I} p_{i}\left(\sigma_{h}^{2}+\sigma_{v_{i}}^{2}\right) .
$$

We assume that the individual sensor nodes transmit over orthogonal channels (e.g., using TDMA, FDMA, or CDMA) so that the signals received by the fusion center are given by

$$
r_{i}=\sqrt{\gamma_{i}} \sqrt{p_{i}}\left(h\left(x_{i}, y_{i}\right)+v_{i}\right)+w_{i}=\sqrt{\gamma_{i} p_{i}} h\left(x_{i}, y_{i}\right)+z_{i} .
$$

Here, $\gamma_{i}$ is the channel gain from sensor node $i$ to the fusion center, which will be used in the following to model Gaussian channels and flat Rayleigh fading channels ${ }^{2}$. For a Gaussian channel, all channel power gains $\gamma_{i}=1$, whereas for Rayleigh fading, the channel power gains $\gamma_{i}$ are exponentially distributed random variables with mean $\mu_{\gamma}$, i.e., the pdf of the channel power gains is ${ }^{3}$

$$
f\left(\gamma_{i}\right)=\frac{1}{\mu_{\gamma}} \mathrm{e}^{-\frac{\gamma_{i}}{\mu_{\gamma}}} .
$$

\footnotetext{
${ }^{2}$ Not that this transmission model assumes pairwise synchronization between transmitter and receiver.

${ }^{3}$ No notational distinction is made between the random variable and its realization.
} 
Furthermore, $w_{i}$ is white receiver noise with variance $\sigma_{w}^{2}$ and

$$
z_{i}=\sqrt{\gamma_{i} p_{i}} v_{i}+w_{i}
$$

denotes the aggregated noise with variance

$$
\sigma_{z_{i}}^{2}=\gamma_{i} p_{i} \sigma_{v_{i}}^{2}+\sigma_{w}^{2}
$$

We next formulate the system model using matrices and vectors. To this end, let $\left(k_{0}, l_{0}\right)$ and $\left(k_{1}, l_{1}\right)$ denote the smallest and largest indices in $\mathbb{A}$, respectively, such that $J=K L$ with $K \triangleq k_{1}-k_{0}+1$ and $L \triangleq\left(l_{1}-l_{0}+1\right)$. We define the block-banded $I \times J$ matrix $\mathbf{G}$ with elements

$$
[\mathbf{G}]_{i, p}=b_{N}\left(x_{i}-k_{p}, y_{i}-l_{p}\right),
$$

the length $I$ vectors $\mathbf{r}, \mathbf{z}$, and length $J$ vector $\mathbf{c}$ with elements

$$
[\mathbf{r}]_{i}=r_{i}, \quad[\mathbf{z}]_{i}=z_{i}, \text { and }[\mathbf{c}]_{p}=c_{k_{p}, l_{p}}
$$

where $k_{p}=k_{0}+((p-1) \bmod K), l_{p}=l_{0}+\left\lfloor\frac{p-1}{K}\right\rfloor$, and $\lfloor p\rfloor$ denotes the largest integer not greater than $\mathrm{p}$. Accordingly, the receive signals in (4.1) can be rewritten in matrix-vector form as

$$
\mathbf{r}=\mathbf{A G c}+\mathbf{z}=\tilde{\mathbf{G}} \mathbf{c}+\mathbf{z}
$$

where

$$
\begin{gathered}
\mathbf{A} \triangleq \operatorname{diag}\left\{\sqrt{\gamma_{i} p_{i}}\right\}, \\
\tilde{\mathbf{G}} \triangleq \mathbf{A G},
\end{gathered}
$$

and the aggregate noise vector $\mathbf{z}$ has covariance matrix (see (4.2))

$$
\mathbf{C}_{\mathbf{z}}=\operatorname{diag}\left\{\gamma_{i} p_{i} \sigma_{v_{i}}^{2}+\sigma_{w}^{2}\right\}
$$

\subsection{Field Reconstruction and Reconstruction Performance}

We determine the field coefficients $\mathbf{c}$ in the linear system model (4.3) using the Best Linear Unbiased Estimator (BLUE) (which for our assumptions equals the Weighted Least Squares (WLS) estimator, see Section 2.2.1) with the noise covariance matrix $\mathbf{C}_{\mathbf{z}}$ as weight, i.e.,

$$
\hat{\mathbf{c}} \triangleq \arg \min _{\mathbf{c}}\|\tilde{\mathbf{G}} \mathbf{c}-\mathbf{r}\|_{\mathbf{C}_{\mathbf{z}}^{-1}}^{2}=\left(\tilde{\mathbf{G}}^{T} \mathbf{C}_{\mathbf{z}}^{-1} \tilde{\mathbf{G}}\right)^{-1} \tilde{\mathbf{G}}^{T} \mathbf{C}_{\mathbf{z}}^{-1} \mathbf{r} .
$$

Note, that in contrast to the reconstruction in Chapter 3, the computation of the coefficient estimates $\hat{\mathbf{c}}$ requires not only the matrix $\tilde{\mathbf{G}}$ (i.e., the sensor node positions and channel gains) but also the noise statistics $\mathbf{C}_{\mathbf{z}}$ to be known at the fusion center. For a solution to exist, the matrix $\tilde{\mathbf{G}}$ must have full rank, which in turn requires $I \geq J$. Technically, the sensor node positions $\left(x_{i}, y_{i}\right)$ have to form a stable sampling set (see Section 3.6.2). 
With the optimal coefficient estimates (4.4), the field can be reconstructed for $(x, y) \in \mathcal{A}$ according to (3.6). To assess the quality of the field reconstruction, we next derive the mean square field reconstruction error $\varepsilon$ within $\mathcal{A}$. (Note that this is the generalization of Equation (3.14) to the 2-D case.)

$$
\begin{aligned}
\varepsilon & =\mathrm{E}\left\{\iint_{\mathcal{A}}(\hat{h}(x, y)-h(x, y))^{2} d x d y\right\}=\mathrm{E}\left\{\iint_{\mathcal{A}}\left((\hat{\mathbf{c}}-\mathbf{c})^{T} \mathbf{g}(x, y)\right)^{2} d x d y\right\} \\
& =\iint_{\mathcal{A}} \mathbf{g}^{T}(x, y) \mathrm{E}\left\{(\hat{\mathbf{c}}-\mathbf{c})(\hat{\mathbf{c}}-\mathbf{c})^{T}\right\} \mathbf{g}(x, y) d x d y=\iint_{\mathcal{A}} \operatorname{tr}\left\{\mathbf{C}_{\hat{\mathbf{c}}-\mathbf{c}} \mathbf{g}(x, y) \mathbf{g}^{T}(x, y)\right\} d x d y \\
& =\operatorname{tr}\left\{\mathbf{C}_{\hat{\mathbf{c}}-\mathbf{c}} \iint_{\mathcal{A}} \mathbf{g}(x, y) \mathbf{g}^{T}(x, y) d x d y\right\}=\operatorname{tr}\left\{\mathbf{C}_{\hat{\mathbf{c}}-\mathbf{c}} \mathbf{G}_{\mathbf{g}}\right\} .
\end{aligned}
$$

Here, the expectation was taken with respect to the noise and we used the length- $J$ vector $\mathbf{g}(x, y)$ defined via its elements

$$
[\mathbf{g}]_{p}(x, y)=g\left(x-k_{p}, y-l_{p}\right),
$$

with $k_{p}$ and $l_{p}$ as in Section 4.2. Furthermore,

$$
\mathbf{G}_{\mathbf{g}}=\iint_{\mathcal{A}} \mathbf{g}(x, y) \mathbf{g}^{T}(x, y) d x d y
$$

denotes the Gramian of $\mathbf{g}(x, y)$ and

$$
\mathbf{C}_{\hat{\mathbf{c}}-\mathbf{c}}=\mathrm{E}\left\{(\hat{\mathbf{c}}-\mathbf{c})(\hat{\mathbf{c}}-\mathbf{c})^{T}\right\}
$$

is the correlation matrix of the field coefficient error $\hat{\mathbf{c}} \mathbf{-} \mathbf{c}$. When using Basis-splines (B-splines) as generator functions, the Gramian $\mathbf{G}_{\mathrm{g}}$ can be shown to be a symmetric block-banded Toeplitz matrix ${ }^{4}$, where the band structure results from the compact support of the B-spline functions and their bandwidth depends on the size of the support. For our system architecture, the correlation matrix (which is equal to the covariance matrix here because $\mathbf{z}$ is zero-mean) of the estimation error can be developed as

$$
\mathbf{C}_{\hat{\mathbf{c}}-\mathbf{c}}=\operatorname{cov}\left\{\left(\tilde{\mathbf{G}}^{T} \mathbf{C}_{\mathbf{z}}^{-1} \tilde{\mathbf{G}}\right)^{-1} \tilde{\mathbf{G}}^{T} \mathbf{C}_{\mathbf{z}}^{-1} \mathbf{z}\right\}=\left(\tilde{\mathbf{G}}^{T} \mathbf{C}_{\mathbf{z}}^{-1} \tilde{\mathbf{G}}\right)^{-1}=\left(\mathbf{G}^{T} \mathbf{A}^{T} \mathbf{C}_{\mathbf{z}}^{-1} \mathbf{A} \mathbf{G}\right)^{-1}=\left(\mathbf{G}^{T} \mathbf{D} \mathbf{G}\right)^{-1} .
$$

Here, we used the diagonal matrix

$$
\mathbf{D} \triangleq \mathbf{A}^{T} \mathbf{C}_{\mathbf{z}}^{-1} \mathbf{A}=\operatorname{diag}\left\{d_{i}\right\}
$$

with

$$
d_{i} \triangleq \frac{1}{\sigma_{v_{i}}^{2}+\frac{\sigma_{w}^{2}}{\gamma_{i} p_{i}}} .
$$

Inserting (4.6) and (4.7) into (4.5), we finally obtain the MSE as

$$
\varepsilon=\operatorname{tr}\left\{\mathbf{C}_{\hat{\mathbf{c}}-\mathbf{c}} \mathbf{G}_{\mathbf{g}}\right\}=\operatorname{tr}\left\{\left(\mathbf{G}^{T} \mathbf{D} \mathbf{G}\right)^{-1} \mathbf{G}_{\mathbf{g}}\right\} .
$$

This expression captures the dependence of the reconstruction MSE on the channel gains $\gamma_{i}$, the sensor node positions $\left(x_{i}, y_{i}\right)$, the AF power scaling factors $p_{i}$, and the noise variances $\sigma_{v_{i}}^{2}$ and $\sigma_{w}^{2}$.

\footnotetext{
${ }^{4}$ For a Toeplitz matrix $\mathbf{A}$ we have $[\mathbf{A}]_{i, j}=[\mathbf{A}]_{i-1, j-1}$. A symmetric block-banded Toeplitz matrix is a symmetric Toeplitz matrix consisting of blocks that are banded and have Toeplitz structure as well.
} 


\subsection{Optimal Power Allocation: Minimization of Mean Square Error}

\subsubsection{General Case}

We aim at allocating the power scaling factors $p_{i}$ such that the reconstruction MSE in (4.8) is minimized subject to a transmit sum-power constraint. Defining the length- $I$ vectors $\mathbf{p}=\left(p_{1} \ldots p_{I}\right)^{T}$ and $\mathbf{q}=\left(q_{1} \ldots q_{I}\right)^{T}$ with $q_{i}=\sigma_{h}^{2}+\sigma_{v_{i}}^{2}$ allows us to write the transmit sum power as

$$
\sum_{i=1}^{I} p_{i}\left(\sigma_{h}^{2}+\sigma_{v_{i}}^{2}\right)=\mathbf{p}^{T} \mathbf{q} .
$$

Recalling that $P_{\mathrm{T}}$ is the maximal transmit sum power, we thus have

$$
\begin{array}{ll}
\underset{\mathbf{p} \in \mathbb{R}_{+}^{I}}{\operatorname{minimize}} & \operatorname{tr}\left\{\left(\mathbf{G}^{T} \mathbf{D}(\mathbf{p}) \mathbf{G}\right)^{-1} \mathbf{G}_{\mathbf{g}}\right\} \\
\text { subject to } & \mathbf{p}^{T} \mathbf{q} \leq P_{\mathrm{T}},
\end{array}
$$

where we made the dependence of $\mathbf{D}$ on $\mathbf{p}$ explicit by writing $\mathbf{D}(\mathbf{p})$. The power allocated to sensor node $i$ depends on the local measurement noise variance $\sigma_{v_{i}}^{2}$, the channel gain $\gamma_{i}$, and (through the matrix $\mathbf{G}$ ) the sensor node positions.

The optimization problem (4.9) is a variation of the well-known A-optimal convex problem (see Appendix 4.A for more details). While in general this problem has no closed-form solution, it can be solved numerically in an efficient manner.

\subsubsection{Critical Sampling}

In some applications the numerical solution of the power allocation problem may still be too expensive for a low-complexity and low-energy fusion center. Thus, we next study in more detail the special case of critical sampling, i.e., $I=J$, for which we show that the power allocation problem has a closed-form solution. We assume that the $I=J$ sensor nodes form a stable sampling set such that $\mathbf{G}$ and $\mathbf{D}$ are square matrices. This allows us to simplify (4.8) as

$$
\begin{aligned}
\varepsilon & =\operatorname{tr}\left\{\left(\mathbf{G}^{T} \mathbf{D} \mathbf{G}\right)^{-1} \mathbf{G}_{\mathbf{g}}\right\}=\operatorname{tr}\left\{\mathbf{G}^{-1} \mathbf{D}^{-1}\left(\mathbf{G}^{T}\right)^{-1} \mathbf{G}_{\mathbf{g}}\right\}=\operatorname{tr}\left\{\mathbf{D}^{-1}\left(\mathbf{G}^{T}\right)^{-1} \mathbf{G}_{\mathbf{g}} \mathbf{G}^{-1}\right\} \\
& =\sum_{i=1}^{I} \frac{1}{d_{i}} g_{i}=\sum_{i=1}^{I} \sigma_{v_{i}}^{2} g_{i}+\sum_{i=1}^{I} \frac{\sigma_{w}^{2} g_{i}}{\gamma_{i} p_{i}},
\end{aligned}
$$

where we defined $g_{i} \triangleq\left[\left(\mathbf{G}^{T}\right)^{-1} \mathbf{G}_{\mathbf{g}} \mathbf{G}^{-1}\right]_{i i}$ and used that $\mathbf{D}^{-1}=\operatorname{diag}\left\{d_{i}^{-1}\right\}$ with $d_{i}^{-1}=\left(\sigma_{v_{i}}^{2}+\frac{\sigma_{w}^{2}}{\gamma_{i} p_{i}}\right)$ (see (4.7)). The first sum in (4.10) is independent of the power scaling factors $p_{i}$ and can therefore not be influenced by the power allocation. It therefore represents a lower bound on the MSE, i.e.,

$$
\varepsilon \geq \lim _{\substack{p_{i} \rightarrow \infty \\ i=1, \ldots, I}} \varepsilon=\sum_{i=1}^{I} \sigma_{v_{i}}^{2} g_{i},
$$


that cannot be attained under the constraint of limited transmit sum power. Since the first sum in (4.10) is independent of the power scaling factors $p_{i}$, the optimization problem in (4.9) simplifies to

$$
\begin{array}{ll}
\underset{\mathbf{p} \in \mathbb{R}_{+}^{J}}{\operatorname{minimize}} & \sum_{i=1}^{J} \frac{\sigma_{w}^{2} g_{i}}{\gamma_{i} p_{i}} \\
\text { subject to } & \mathbf{p}^{T} \mathbf{q} \leq P_{\mathrm{T}} .
\end{array}
$$

The Lagrangian associated to this problem equals

$$
L(\mathbf{p}, \lambda)=\sum_{i=1}^{I} \frac{\sigma_{w}^{2} g_{i}}{\gamma_{i} p_{i}}+\lambda\left(\mathbf{p}^{T} \mathbf{q}-P_{\mathrm{T}}\right)=\sum_{i=1}^{I}\left(\frac{\sigma_{w}^{2} g_{i}}{\gamma_{i} p_{i}}+\lambda p_{i} q_{i}\right)-\lambda P_{\mathrm{T}}
$$

and the Lagrangian dual function reads

$$
\begin{aligned}
g(\lambda) & =L\left(\mathbf{p}^{\star}, \lambda\right)=\inf _{\mathbf{p}} L(\mathbf{p}, \lambda)=\inf _{\mathbf{p}} \sum_{i=1}^{J}\left(\frac{\sigma_{w}^{2} g_{i}}{\gamma_{i} p_{i}}+\lambda p_{i} u_{i}\right)-\lambda P_{\mathrm{T}}=\sum_{i=1}^{I} \inf _{p_{i}}\left(\frac{\sigma_{w}^{2} g_{i}}{\gamma_{i} p_{i}}+\lambda p_{i} q_{i}\right)-\lambda P_{\mathrm{T}} \\
& =\sum_{i=1}^{I}\left(\frac{\sigma_{w}^{2} g_{i}}{\gamma_{i} p_{i}^{\star}}+\lambda p_{i}^{\star} q_{i}\right)-\lambda P_{\mathrm{T}}=2 \sigma_{w} \sqrt{\lambda} \sum_{i=1}^{I} \sqrt{\frac{g_{i} q_{i}}{\gamma_{i}}}-\lambda P_{\mathrm{T}},
\end{aligned}
$$

where

$$
p_{i}^{\star}=\arg \inf _{p_{i}}\left(\frac{\sigma_{w}^{2} g_{i}}{\gamma_{i} p_{i}}+\lambda p_{i} q_{i}\right)=\sqrt{\frac{\sigma_{w}^{2} g_{i}}{\lambda \gamma_{i} q_{i}}} .
$$

We therefore have the Lagrange dual problem

$$
\begin{array}{ll}
\underset{\lambda}{\operatorname{maximize}} & 2 \sigma_{w} \sqrt{\lambda} \sum_{i=1}^{I} \sqrt{\frac{g_{i} q_{i}}{\gamma_{i}}}-\lambda P_{\mathrm{T}} \\
\text { subject to } & \lambda \geq 0
\end{array}
$$

whose solution is given by

$$
\lambda^{\star}=\frac{\sigma_{w}^{2}\left(\sum_{i=1}^{I} \sqrt{\frac{g_{i} q_{i}}{\gamma_{i}}}\right)^{2}}{P_{\mathrm{T}}^{2}} .
$$

For our convex problem, the Karush-Kuhn-Tucker (KKT) conditions [10, Chapter 5] hold, which is a sufficient condition for strong duality. With the dual optimal solution $\lambda^{\star}$, the optimal solution for the primal problem equals $L\left(\mathbf{p}^{\star}, \lambda^{\star}\right)=g\left(\lambda^{\star}\right)$ with the primal feasible minimizer

$$
p_{i}^{\star}=P_{\mathrm{T}} \frac{\sqrt{\frac{g_{i}}{\gamma_{i} q_{i}}}}{\sum_{j=1}^{I} \sqrt{\frac{g_{j} q_{j}}{\gamma_{j}}}}=P_{\mathrm{T}} \frac{\sqrt{\frac{g_{i}}{\gamma_{i}\left(\sigma_{h}^{2}+\sigma_{v_{i}}^{2}\right)}}}{\sum_{j=1}^{I} \sqrt{\frac{g_{j}\left(\sigma_{h}^{2}+\sigma_{v_{j}}^{2}\right)}{\gamma_{j}}}} .
$$

It can be seen from (4.12) that every sensor node is allocated non-zero power. This ensures that the stable sampling set is preserved. With the definitions

$$
\alpha_{i} \triangleq \frac{\beta_{i}}{\sum_{j=1}^{I} \beta_{j}}
$$


and

$$
\beta_{i} \triangleq \sqrt{\frac{g_{i}\left(\sigma_{h}^{2}+\sigma_{v_{i}}^{2}\right)}{\gamma_{i}}}
$$

the optimal sensor node transmit powers can be written as

$$
P_{i}^{\star}=p_{i}^{\star}\left(\sigma_{h}^{2}+\sigma_{v_{i}}^{2}\right)=\alpha_{i} P_{\mathrm{T}} .
$$

Here, $\alpha_{i}$ is the fraction of power used by sensor node $i$. This fraction is large if $g_{i}$ is large, which means that sensor node $i$ is rather isolated, or if the associated channel gain $\gamma_{i}$ is small. Hence, the optimal power allocation compensates for poor channel or sampling conditions. It is worth noting that the power fraction $\alpha_{i}$ becomes large also when the associated measurement noise variance $\sigma_{v_{i}}^{2}$ is large. As can be verified from (4.12), the optimal power scaling $p_{i}^{\star}$ in this case is small, i.e., the large transmit power is solely due to the fact that the power of the measurement is already large.

\subsection{Optimal Power Allocation: Minimization of Transmit Sum Power}

\subsubsection{General Case}

We aim at allocating the power scaling factors $p_{i}$ such that the transmit sum power is minimized subject to an MSE target in (4.8). With $\mathbf{p}$ and $\mathbf{q}$ as defined in Section 4.4.1 we thus have

$$
\begin{array}{ll}
\underset{\mathbf{p} \in \mathbb{R}_{+}^{I}}{\operatorname{minimize}} & \mathbf{p}^{T} \mathbf{q} \\
\text { subject to } & \operatorname{tr}\left\{\left(\mathbf{G}^{T} \mathbf{D}(\mathbf{p}) \mathbf{G}\right)^{-1} \mathbf{G}_{\mathbf{g}}\right\} \leq \varepsilon_{\max },
\end{array}
$$

where we made the dependence of $\mathbf{D}$ on $\mathbf{p}$ explicit by writing $\mathbf{D}(\mathbf{p})$. The power allocated to sensor node $i$ depends on the local measurement noise variance $\sigma_{v_{i}}^{2}$, the channel gain $\gamma_{i}$, and (through the matrix $\mathbf{G}$ ) the sensor node positions. The optimization problem is convex and has no closed-form solution in general, but it can be solved numerically in an efficient manner.

\subsubsection{Critical Sampling}

As in Section 4.4.2, we study the special case of critical sampling in more detail again, for which we show that the power allocation problem has a closed-form solution. With the assumption of $I=J$ we use the expression for the MSE from (4.10). Then the optimization problem in (4.15) simplifies to

$$
\begin{array}{ll}
\underset{\mathbf{p} \in \mathbb{R}_{+}^{J}}{\operatorname{minimize}} & \mathbf{p}^{T} \mathbf{q} \\
\text { subject to } & \sum_{i=1}^{I} \sigma_{v_{i}}^{2} g_{i}+\sum_{i=1}^{I} \frac{\sigma_{w}^{2} g_{i}}{\gamma_{i} p_{i}} \leq \varepsilon_{\max } .
\end{array}
$$

Note that the MSE target $\varepsilon_{\max }$ cannot be chosen arbitrarily small. In fact, even with infinite transmit powers the MSE is lower bounded by $\sum_{i=1}^{I} \sigma_{v_{i}}^{2} g_{i}$. Hence the MSE target has to be large enough to ensure

$$
\varepsilon_{\max }^{\prime} \triangleq \varepsilon_{\max }-\sum_{i=1}^{I} \sigma_{v_{i}}^{2} g_{i}>0
$$


to yield a solution to the optimization problem in (4.16). Here, $\varepsilon_{\max }^{\prime}$ is the part of the MSE target affected by the power allocation.

The Lagrangian associated to the problem (4.16) equals

$$
L(\mathbf{p}, \lambda)=\mathbf{p}^{T} \mathbf{q}+\lambda\left(\sum_{i=1}^{I} \frac{\sigma_{w}^{2} g_{i}}{\gamma_{i} p_{i}}-\varepsilon_{\max }^{\prime}\right)=\sum_{i=1}^{I}\left(p_{i} q_{i}+\lambda \frac{\sigma_{w}^{2} g_{i}}{\gamma_{i} p_{i}}\right)-\lambda \varepsilon_{\max }^{\prime}
$$

and the Lagrangian dual function reads

$$
\begin{aligned}
g(\lambda) & =L\left(\mathbf{p}^{\star}, \lambda\right)=\inf _{\mathbf{p}} L(\mathbf{p}, \lambda)=\inf _{\mathbf{p}} \sum_{i=1}^{I}\left(p_{i} q_{i}+\lambda \frac{\sigma_{w}^{2} g_{i}}{\gamma_{i} p_{i}}\right)-\lambda \varepsilon_{\max }^{\prime} \\
& =\sum_{i=1}^{I} \inf _{p_{i}}\left(p_{i} q_{i}+\lambda \frac{\sigma_{w}^{2} g_{i}}{\gamma_{i} p_{i}}\right)-\lambda \varepsilon_{\max }^{\prime}=\sum_{i=1}^{I}\left(p_{i}^{\star} q_{i}+\lambda \frac{\sigma_{w}^{2} g_{i}}{\gamma_{i} p_{i}^{\star}}\right)-\lambda \varepsilon_{\max }^{\prime} \\
& =2 \sigma_{w} \sqrt{\lambda} \sum_{i=1}^{I} \sqrt{\frac{g_{i} q_{i}}{\gamma_{i}}}-\lambda \varepsilon_{\max }^{\prime},
\end{aligned}
$$

where

$$
p_{i}^{\star}=\arg \inf _{p_{i}}\left(p_{i} q_{i}+\lambda \frac{\sigma_{w}^{2} g_{i}}{\gamma_{i} p_{i}}\right)=\sqrt{\frac{\lambda \sigma_{w}^{2} g_{i}}{\gamma_{i} q_{i}}} .
$$

We therefore have the Lagrange dual problem

$$
\begin{aligned}
& \underset{\lambda}{\operatorname{maximize}} 2 \sigma_{w} \sqrt{\lambda} \sum_{i=1}^{I} \sqrt{\frac{g_{i} q_{i}}{\gamma_{i}}}-\lambda \varepsilon_{\max }^{\prime} \\
& \text { subject to } \quad \lambda \geq 0
\end{aligned}
$$

whose solution is given by

$$
\lambda^{\star}=\frac{\sigma_{w}^{2}\left(\sum_{i=1}^{I} \sqrt{\frac{g_{i} q_{i}}{\gamma_{i}}}\right)^{2}}{\left(\varepsilon_{\max }^{\prime}\right)^{2}} .
$$

Again, it can be shown that the KKT conditions [10, Chapter 5] hold, so that strong duality is fulfilled. With the dual optimal solution $\lambda^{\star}$, the optimal solution for the primal problem equals $L\left(\mathbf{p}^{\star}, \lambda^{\star}\right)=g\left(\lambda^{\star}\right)$ with the primal feasible minimizer

$$
p_{i}^{\star}=\frac{\sigma_{w}^{2}}{\varepsilon_{\max }^{\prime}} \sqrt{\frac{g_{i}}{\gamma_{i} q_{i}}} \sum_{j=1}^{I} \sqrt{\frac{g_{j} q_{j}}{\gamma_{j}}}=\frac{\sigma_{w}^{2}}{\varepsilon_{\max }^{\prime}} \sqrt{\frac{g_{i}}{\gamma_{i}\left(\sigma_{h}^{2}+\sigma_{v_{i}}^{2}\right)}} \sum_{j=1}^{I} \sqrt{\frac{g_{j}\left(\sigma_{h}^{2}+\sigma_{v_{j}}^{2}\right)}{\gamma_{j}}} .
$$

The optimal sensor node transmit powers can then be written as

$$
P_{i}^{\star}=p_{i}^{\star}\left(\sigma_{h}^{2}+\sigma_{v_{i}}^{2}\right)=\frac{\sigma_{w}^{2}}{\varepsilon_{\max }^{\prime}} \sqrt{\frac{g_{i}\left(\sigma_{h}^{2}+\sigma_{v_{i}}^{2}\right)}{\gamma_{i}}} \sum_{j=1}^{I} \sqrt{\frac{g_{j}\left(\sigma_{h}^{2}+\sigma_{v_{j}}^{2}\right)}{\gamma_{j}}} .
$$

Inserting (4.17) into (4.10) and with the definitions of $\alpha_{i}$ from (4.13) and $\beta_{i}$ from (4.14), we get for the optimal MSE

$$
\varepsilon^{\star}=\sum_{i=1}^{I} \sigma_{v_{i}}^{2} g_{i}+\sum_{i=1}^{I} \frac{\sigma_{w}^{2} g_{i}}{\gamma_{i} p_{i}^{\star}}=\sum_{i=1}^{I}\left(\sigma_{v_{i}}^{2} g_{i}+\alpha_{i} \varepsilon_{\max }^{\prime}\right)
$$




$$
=\sum_{i=1}^{I}\left(\sigma_{v_{i}}^{2} g_{i}+\alpha_{i}\left(\varepsilon_{\max }-\sum_{j=1}^{I} \sigma_{j}^{2} g_{j}\right)\right)=\sum_{i=1}^{I} \varepsilon_{i}=\varepsilon_{\max },
$$

i.e., the optimal power allocation achieves the MSE target $\varepsilon_{\max }$ with equality. Here, in the critical sampling case, the overall MSE can be split up into the contributions $\varepsilon_{i}$ of the individual sensor nodes. Each sensor node contributes a part $\varepsilon_{i}=\sigma_{v_{i}}^{2} g_{i}+\alpha_{i} \varepsilon_{\max }^{\prime}$ to the overall MSE, where the fraction $\alpha_{i}$ of the eligible MSE $\varepsilon_{\max }^{\prime}$ that corresponds to a sensor node is large if $g_{i}$ is large (which means that sensor node $i$ is rather isolated) or if the associated channel gain $\gamma_{i}$ is small. As a result, sensor nodes with the better measurement quality, i.e., the lower $\sigma_{v_{i}}^{2}$, are assigned a larger $p_{i}$, i.e., a smaller transmit power $P_{i}$, than those with poor measurement quality. Also, the placement of the sensor node, reflected by the factor $g_{i}$, plays a role in the power allocation. Those sensor nodes that are necessary to retain a stable sampling set, i.e., those with a large $g_{i}$, are allowed a higher contribution to the overall MSE than others.

\subsection{Particular Cases and Benchmarks}

In the following subsections we mention some special cases which we will either use for comparison in the numerical simulations or which give insight to the mechanisms of power allocation for our field reconstruction scheme.

\subsubsection{Uniform Power Allocation}

In the numerical simulations we will compare the power allocation strategies presented above with a uniform power allocation that assigns the same power to all sensor nodes, regardless of the measurement noise and channel conditions they experience. A uniform power allocation is therefore given by

$$
p_{i}=\frac{P_{\mathrm{T}}}{I\left(\sigma_{h}^{2}+\sigma_{v_{i}}^{2}\right)},
$$

where $P_{\mathrm{T}}$ is the maximal allowed transmit sum power for the sensor nodes. Obviously, a uniform power allocation is not optimal in general but it serves as a benchmark for the strategies presented above and shows the possible performance gains that come with their use.

\subsubsection{Uniform MSE Target}

In contrast to the critical sampling case presented in Section 4.5.2, the overall MSE target cannot be partitioned into separate contributions of the single sensor nodes in the general case. This fact prohibits a uniform assignment of the MSE target to the sensor nodes. In the case of critical sampling though, the MSE target can be split up to a sum of uniform MSE contributions

$$
\varepsilon_{i}=\frac{\varepsilon_{\max }}{J}
$$

The sensor nodes can then calculate their power scaling factor according to

$$
p_{i}=\frac{J \sigma_{w}^{2} g_{i}}{\left(\varepsilon_{\max }-\sum_{i=1}^{J} \sigma_{v_{i}}^{2} g_{i}\right) \gamma_{i}}=\frac{J \sigma_{w}^{2} g_{i}}{\varepsilon_{\max }^{\prime} \gamma_{i}}
$$

thereby meeting the MSE constraint with equality. 


\subsubsection{Gaussian Channels and Identical Noise Variances}

We next make some stringent assumptions that lead to a special case for which it is easier to calculate the MSE and to asses its dependence on the individual parameters. If we assume Gaussian channels, i.e., $\gamma_{i}=1$ for all $i$, identical measurement noise variance $\sigma_{v_{i}}^{2}=\sigma_{v}^{2}$ at all sensor nodes, and uniform power allocation to the sensor nodes, i.e., $p_{i}=\frac{P_{T}}{I\left(\sigma_{h}^{2}+\sigma_{v}^{2}\right)}=\frac{P_{T}^{\prime}}{I}$, it follows that $\mathbf{D}=d \mathbf{I}$ with

$$
d \triangleq \frac{1}{\sigma_{v}^{2}+\frac{\sigma_{w}^{2} I}{P_{T}^{\prime}}},
$$

and therefore (4.8) simplifies to

$$
\begin{aligned}
\varepsilon & =\operatorname{tr}\left\{\left(\mathbf{G}^{T} d \mathbf{I} \mathbf{G}\right)^{-1} \mathbf{G}_{\mathbf{g}}\right\}=\frac{1}{d} \operatorname{tr}\left\{\left(\mathbf{G}^{T} \mathbf{G}\right)^{-1} \mathbf{G}_{\mathbf{g}}\right\} \\
& =\left(\sigma_{v}^{2}+\frac{\sigma_{w}^{2} I}{P_{T}^{\prime}}\right) \operatorname{tr}\left\{\left(\mathbf{G}^{T} \mathbf{G}\right)^{-1} \mathbf{G}_{\mathbf{g}}\right\} .
\end{aligned}
$$

It can be seen that under the above assumptions the MSE is a product of two terms. The first one is completely determined by the measurement and receiver noise and by the per-sensor node transmit power and the second term depends only on the generator function and the sensor node placement (via G). Note that in spite of identical channel gains and noise variances, the uniform power allocation still is suboptimal in this case because it does not take into account the influence of the sensor node positions on the reconstruction error.

Using the same assumptions for the optimal power allocation schemes for the critical sampling case presented above, we get from (4.13)

$$
\alpha_{i}=\frac{\sqrt{g_{i}}}{\sum_{j=1}^{I} \sqrt{g_{j}}} .
$$

Here, the power allocation is not uniform unless all $g_{i}$ are identical, i.e., unless the sensor nodes are regularly placed on an integer lattice with arbitrary offset.

\subsection{Numerical Simulations}

We next present numerical results to illustrate the performance of our power allocation strategies. We consider a shift-invariant space using a B-spline of order $N=3$ and normalize the field such that the average power equals $\sigma_{h}^{2}=1$. The region being sensed is $\mathcal{A}=[0,5] \times[0,5]$, corresponding to a number of $J=36$ field coefficients. We used a WSN deployment with $I \geq 36$ sensor nodes, where the first $J$ sensor nodes (the number for critical sampling) are placed on a square grid and the remaining $I-J$ sensor nodes are randomly placed according to a spatially uniform distribution over the region $\mathcal{A}$. Since the first $J$ sensor nodes form a stable sampling set, the entire WSN of $I$ sensor nodes does so too. The measurement noise variance at the different sensor nodes was chosen according to a uniform distribution, i.e., $\sigma_{v_{i}}^{2}=$ Unif $\{0.01,0.1\}$. This corresponds to measurement SNRs between $10 \mathrm{~dB}$ and $20 \mathrm{~dB}$. For the Gaussian channel case we set the channel gains $\gamma_{i}=1$, whereas for the Rayleigh-fading channel we generated exponentially distributed $\gamma_{i}$ with mean $\mu_{\gamma}=1$. For each scenario, we chose the receiver noise variance as $\sigma_{w}^{2}=$ Unif $\{0.01,0.1\}$, corresponding to an SNR between $10 \mathrm{~dB}$ and $20 \mathrm{~dB}$. 


\subsubsection{Minimization of the Mean Square Error}

We first compare the optimal power allocation schemes minimizing the MSE derived in Section 4.4 with a uniform power allocation. We consider setups with

(a) low transmit sum power of $P_{\mathrm{T}}=10$ and with

(b) high transmit sum power of $P_{\mathrm{T}}=100$.

Using the parameters specified above, our simulations compare three sensor node power allocation schemes:

(i) the numerically evaluated optimal power allocation scheme according to (4.9);

(ii) power allocation according to the closed-form expression (4.12) for the first 36 sensor nodes and zero power for the remaining sensor nodes;

(iii) a baseline scheme with uniform power allocation, i.e., $P_{i}=P_{\mathrm{T}} / I$.

Figure 4.1 displays the normalized field reconstruction MSE $\frac{\varepsilon}{J|\mathcal{A}|}$ versus the number of sensor nodes $I$ for both sum power levels. It is seen that the optimal power allocation performs best for all numbers of sensor nodes and that its performance advantage over the other two schemes rises with increasing $I$. The performance of the power allocation (4.12) for the critical sampling case is independent of $I$ since only the first $J=36$ sensor nodes have non-zero power, i.e., the remaining sensor nodes stay silent. This power allocation is superior to uniform power allocation at low transmit sum power but tends to be inferior to uniform allocation at high transmit sum power, specifically for larger $I$.

Figure 4.2 depicts an example of how the sensor node powers $P_{i}$ are allocated in the case of Gaussian channels and $I=64$. We divide the sensor nodes into two groups (sensors 1-36 and 37-64) and sort the sensor nodes within each group according to increasing noise variance $\sigma_{v_{i}}^{2}$. That way, the impact of the sensor node positions is easier to identify. The power allocation for the critically sampled case (using only sensors 1-36) and low transmit sum power is shown in Figure 4.2(a). Clearly, higher measurement noise power tends to scale up the transmit power (even if the power scaling factor $p_{i}$ is smaller). However, the power increase is not monotonic due to the additional impact of the sensor node position. For low transmit sum power, the optimal power allocation is shown in Figure 4.2(b). The largest part of power is again allocated to sensor nodes 1-36 due to their good localization on a regular grid. In this power-limited regime, only few sensor nodes in the second group (those with favorable position and measurement noise) have non-negligible power. For the high power scenario shown in Figure 4.2(c), power is allocated more evenly to all sensors even though a large fraction of the sum power is still allocated to the first group of sensor nodes.

A similar example for the power allocation is depicted in Figure 4.3. Here, the power is again allocated to the sensor nodes for the case of Gaussian channels and $I=64$, but for equal measurement noise variances $\sigma_{v_{i}}^{2}=$ $\sigma_{v}^{2}$ so that the power allocation only depends on the importance of the sensor nodes' position for maintaining a stable set of sampling for the field reconstruction. The size of the circles marking the sensor node positions corresponds to the fraction of the power $\alpha_{i}$ assigned to the sensor nodes. The power allocation for the critically sampled case (using only sensors 1-36) and low transmit sum power is shown in Figure 4.3(a). All the regularly 


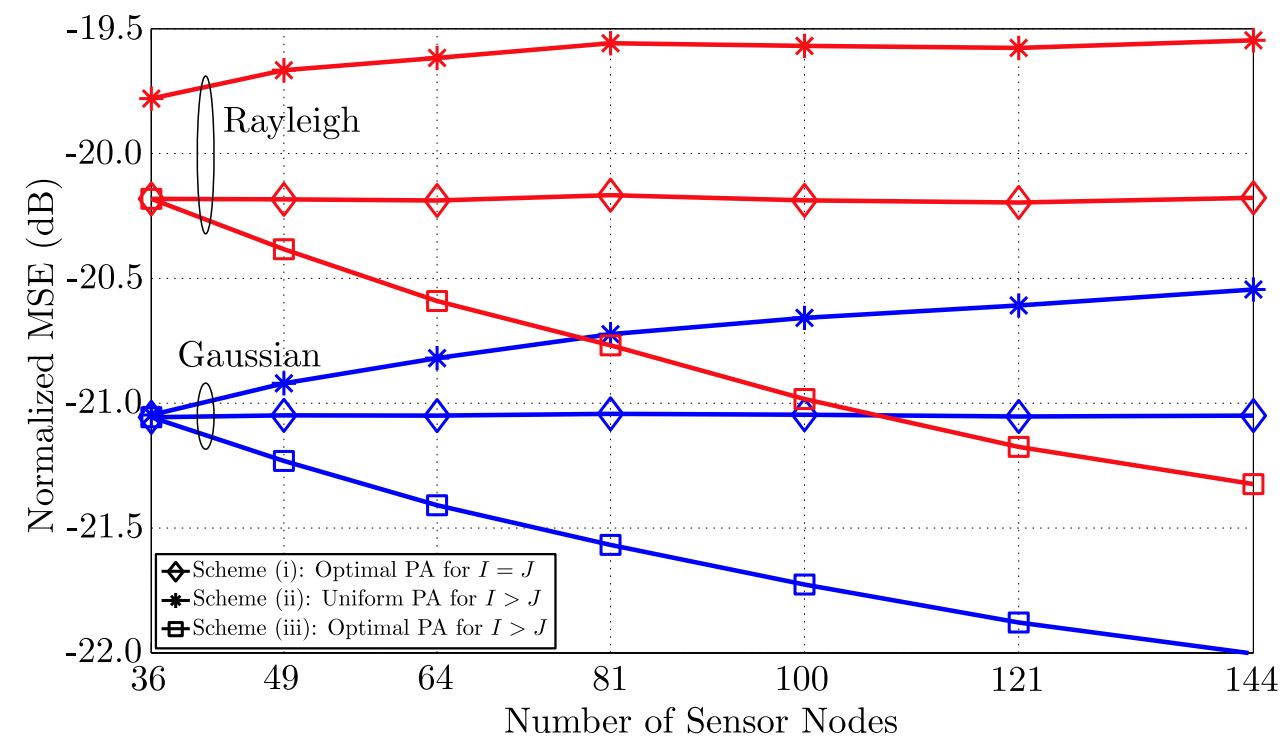

(a)

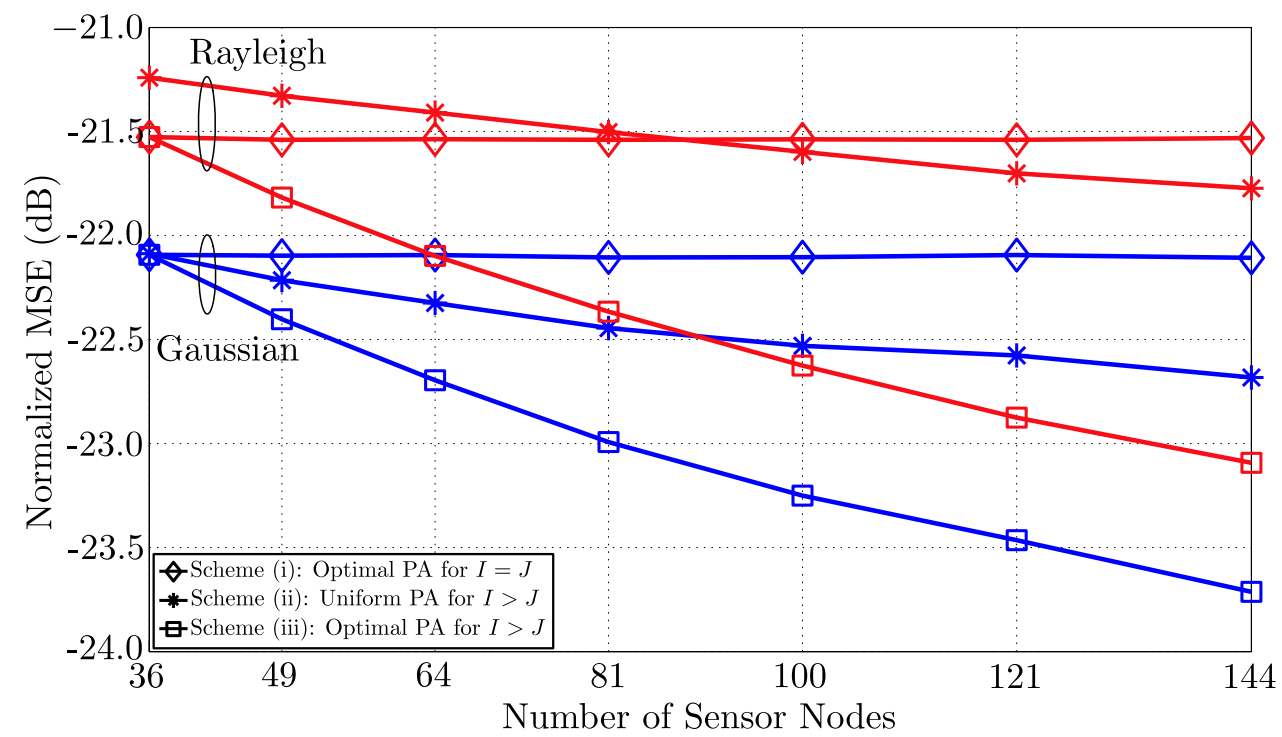

(b)

Figure 4.1: Comparison of the reconstruction performance for various sensor power allocation schemes in terms of normalized MSE versus numbers of sensor nodes I in Gaussian and Rayleigh fading channels: (a) low transmit sum power $P_{T}=10$, (b) high transmit sum power $P_{T}=100$.

placed sensor nodes are allocated the same power as their location is crucial for the reconstruction. The other sensor nodes (visible as small points) are not considered by the allocation scheme. For low transmit sum power, the optimal power allocation is shown in Figure 4.3(b). As in the critical sampling case, the largest part of power is allocated to sensor nodes 1-36 due to their good localization on a regular grid. Only few sensor nodes off the regular grid have non-negligible power. For the high power scenario shown in Figure 4.3(c), power is allocated more evenly to all sensors even though most of the power is still allocated to the sensor nodes on the regular grid. The optimal power allocation scheme establishes stable sets of sampling by not allowing large gaps between neighboring sensor nodes. 


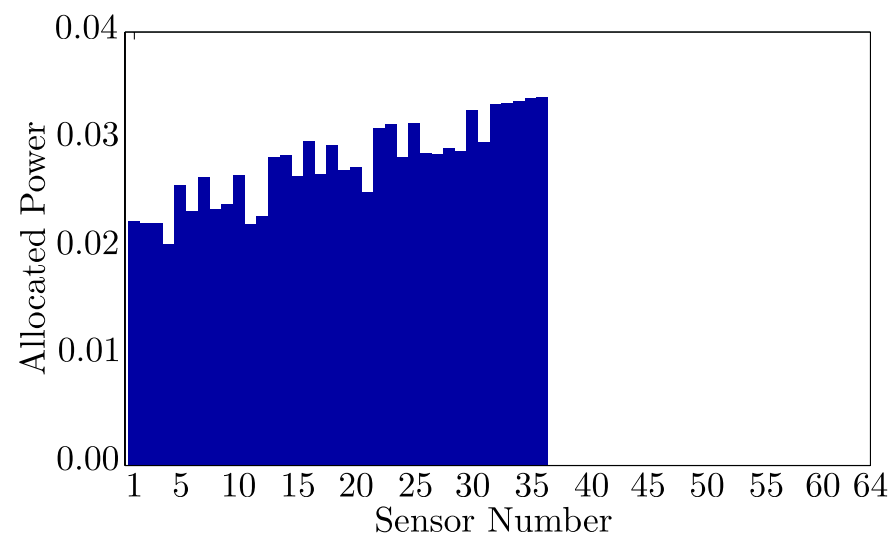

(a)

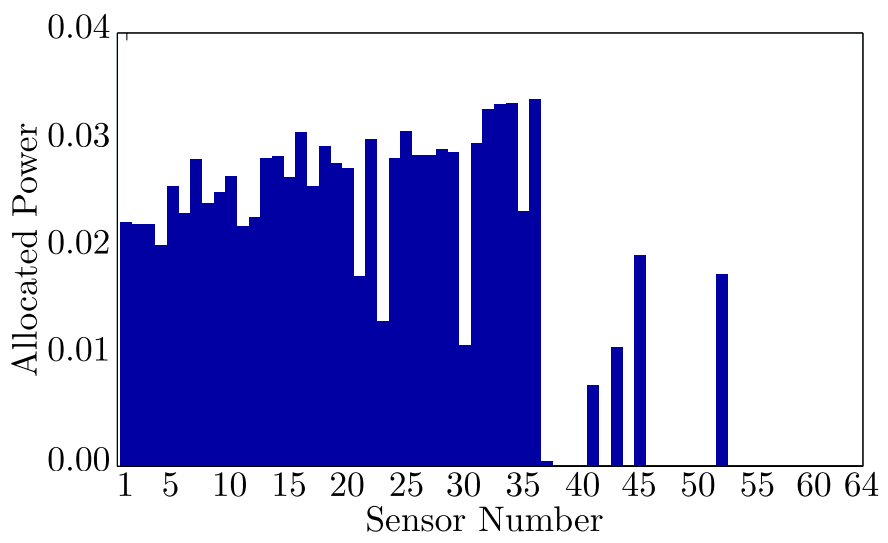

(b)

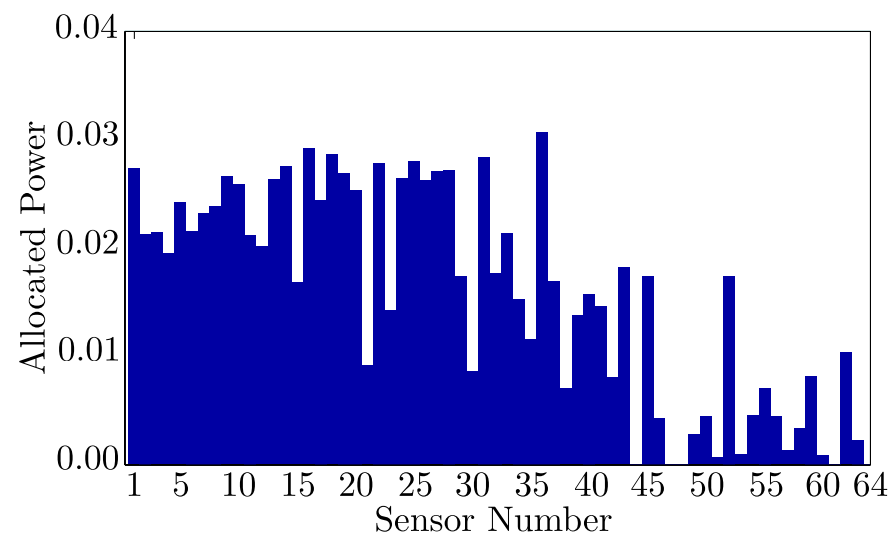

(c)

Figure 4.2: Example for the sensor power allocation in a WSN with $I=64$ sensors and Gaussian channels. The plots display the fraction of power $\alpha_{i}$ versus sensor number for (a) optimal power allocation for the first group of 36 sensors and low transmit sum power $P_{T}=10$; (b) optimal allocation for low transmit sum power $P_{T}=10$, and (c) optimal allocation for high transmit sum power $P_{T}=100$. The sensors within each group (sensors 1-36 and 37-64) are sorted according to increasing noise variance $\sigma_{v_{i}}^{2}$. 


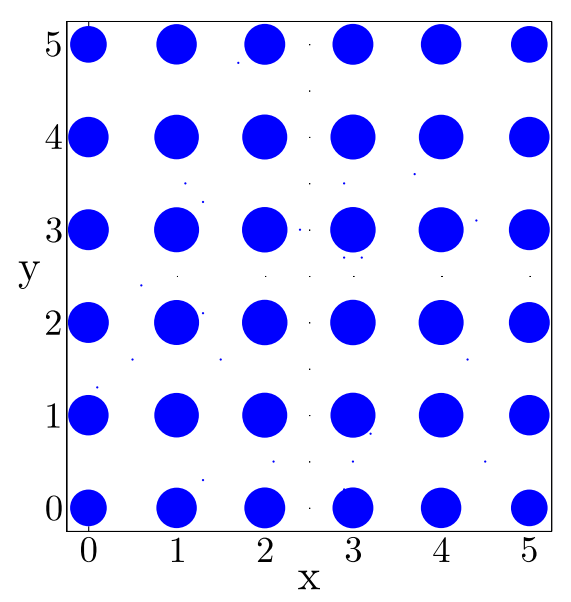

(a)

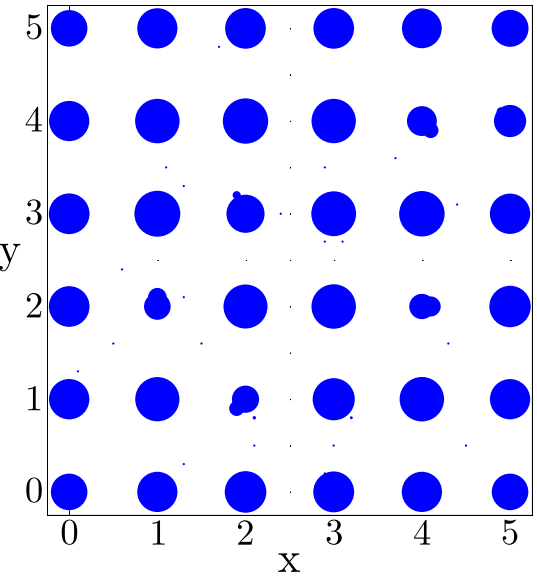

(b)

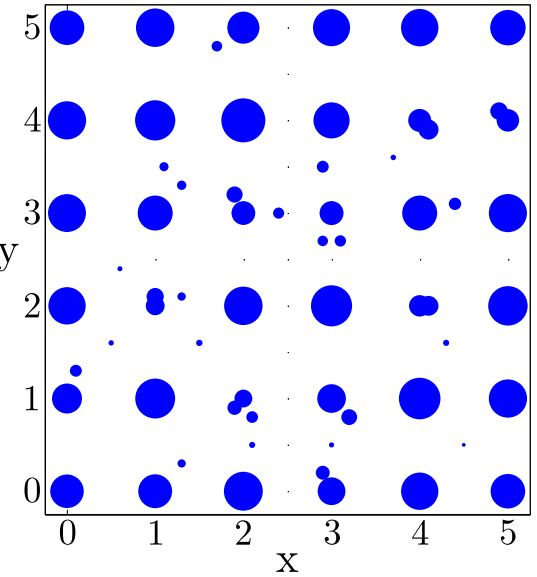

(c)

Figure 4.3: Example for the sensor power allocation in a WSN with $I=64$ sensors, Gaussian channels, and equal measurement noise $\sigma_{v}^{2}$. The plots display the sensor node positions over the region $\mathcal{A}$ for (a) optimal power allocation for the first group of 36 sensor nodes and low transmit sum power $P_{T}=10$; $(b)$ optimal allocation for low transmit sum power $P_{T}=10$, and (c) optimal allocation for high transmit sum power $P_{T}=100$, where the size of the circles marking the sensor node position corresponds to the fraction of power $\alpha_{i}$ assigned to the sensor nodes. As all sensor nodes experience the same measurement noise variance $\sigma_{v}^{2}$, the power allocation is only based on the sensor nodes' positions.

\subsubsection{Minimization of Transmit Power}

We next compare the optimal power allocation schemes minimizing the transmit power derived in Section 4.5. We consider setups with

(a) low normalized MSE target $\frac{\varepsilon_{\max }}{J|\mathcal{A}|}=0.01$ and with

(b) high normalized MSE target $\frac{\varepsilon_{\max }}{J|\mathcal{A}|}=0.1$.

Using the parameters specified above, our simulations compare three sensor node power allocation schemes:

(i) the numerically evaluated optimal power allocation scheme according to (4.15);

(ii) power allocation according to the closed-form expression (4.17) for the first 36 sensor nodes and zero power for the remaining sensor nodes;

(iii) a scheme with uniform power allocation for the first 36 sensor nodes and zero power for the remaining sensor nodes according to (4.19).

Figure 4.4 displays the optimal transmit power versus the number of sensor nodes $I$ for both MSE targets. It is seen that the optimal power allocation performs best for all numbers of sensor nodes and that its performance advantage over the other two schemes rises with increasing $I$. The performance of the power allocation (4.12) is independent of $I$ since only the first $J=36$ sensor nodes have non-zero power, i.e., the remaining sensor nodes stay silent. For Gaussian channels, this power allocation performs identical to uniform power allocation but tends to be superior to uniform allocation for the case of Rayleigh fading channels. 


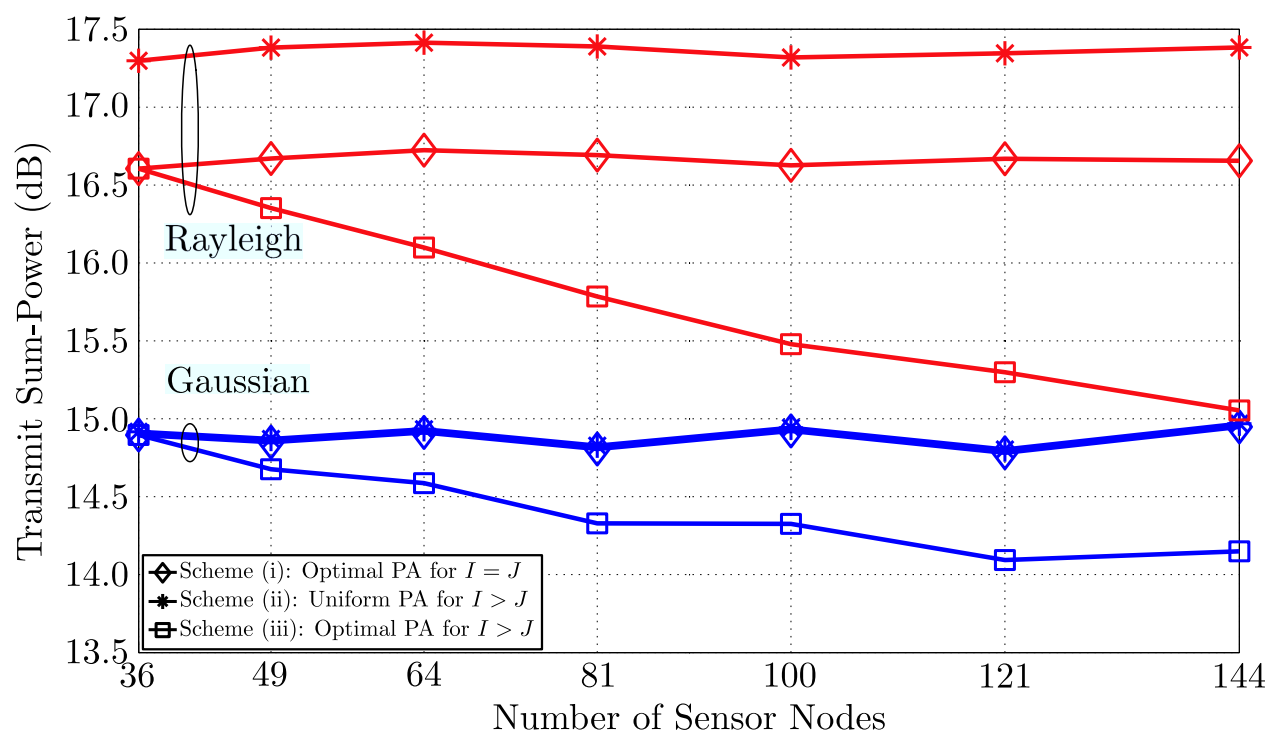

(a)

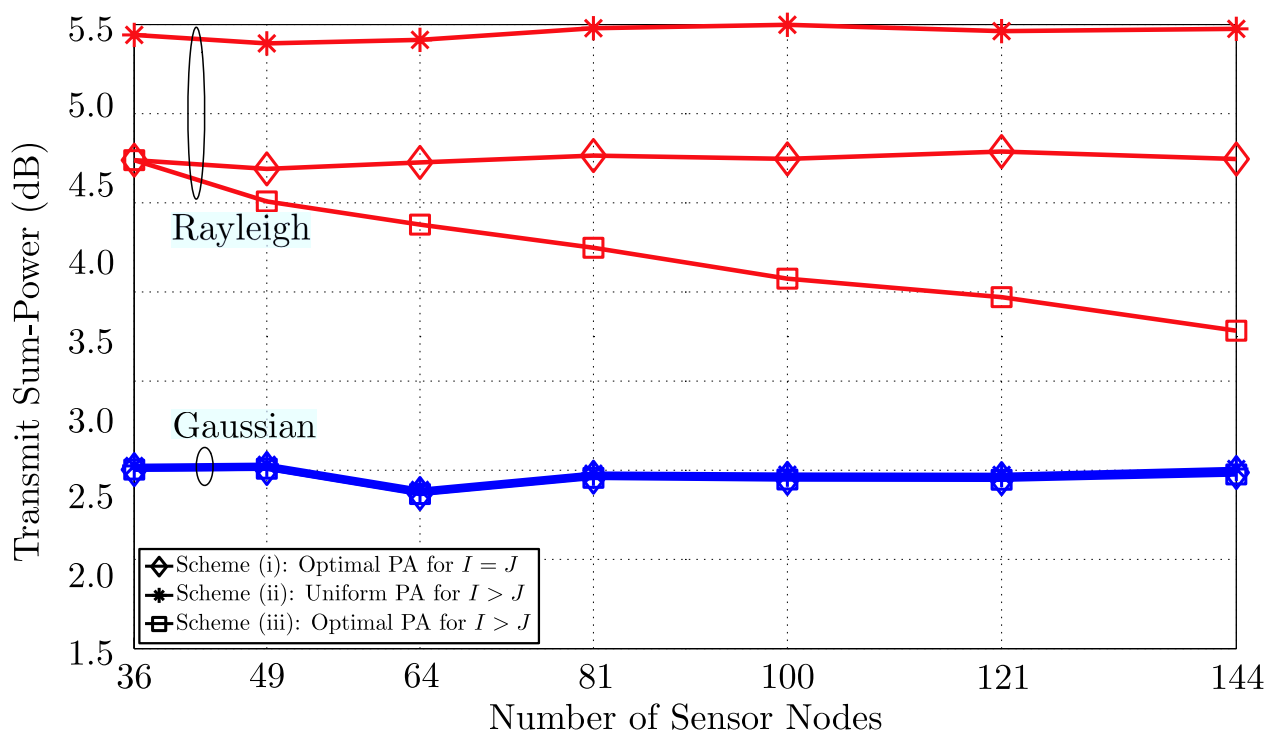

(b)

Figure 4.4: Comparison of the reconstruction performance for various sensor power allocation schemes in terms of transmit sum power $P_{T}$ versus numbers of sensor nodes $I$ in Gaussian and Rayleigh fading channels: (a) small normalized MSE target $\frac{\varepsilon_{\max }}{J|\mathcal{A}|}=0.01$, (b) large normalized MSE target $\frac{\varepsilon_{\max }}{J|\mathcal{A}|}=0.1$.

Figure 4.5 plots the relation between the minimal transmit sum power over the MSE target for the derived power allocation schemes for $I=100$ sensor nodes. For Gaussian channels and large MSE target, all power allocation schemes perform equally and optimally. For small MSE target the performance advantage of the optimal power allocation scheme for all sensor nodes over the other allocation schemes increases with decreasing MSE target. For Rayleigh fading channels and all MSE targets, the optimal power allocation for all sensor nodes outperforms the optimal power allocation for critical sampling. Uniform power allocation shows the weakest performance and requires up to $3 \mathrm{~dB}$ more power compared to the optimal power allocation. 


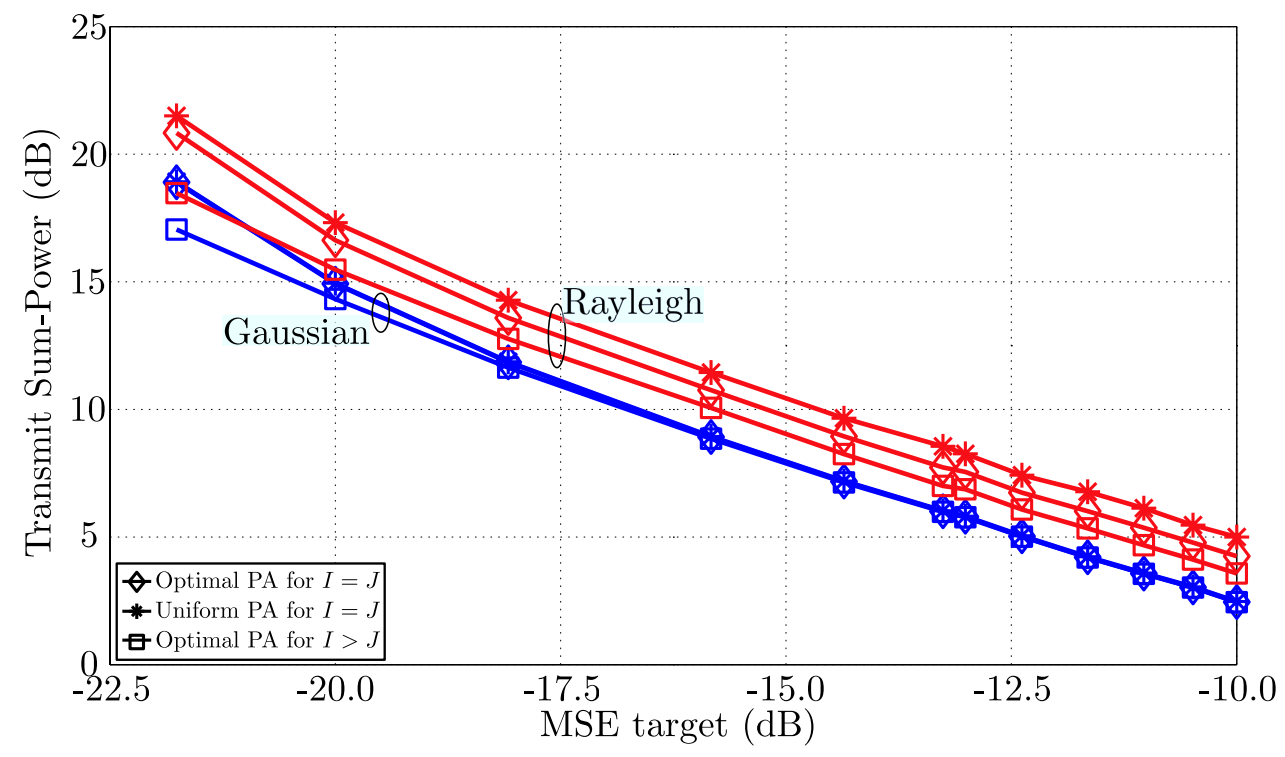

Figure 4.5: Minimal transmit sum power versus MSE target for $I=100$ sensor nodes for Gaussian and Rayleigh fading channels.

\subsection{Conclusions}

We considered field reconstruction in WSN based on shift-invariant spaces and an AF protocol for the transmission of sensor node measurements to the fusion center. We derived the MSE achieved by this scheme and developed both an MSE-optimal and a transmit sum-power-optimal scheme for power allocation to the sensor nodes. For the case of critical sampling, the optimal power allocation was expressed in closed form. Numerical simulations for Gaussian and Rayleigh fading channels demonstrated how power is allocated to the sensor nodes and how this impacts the overall field reconstruction quality.

Applying the optimal power allocation scheme for the critically sampled case by using only $J \leq I$ sensor nodes is clearly suboptimal since many sensor node measurements remain unused. This drawback can be circumvented by splitting the WSN into sub-WSNs of size $J$, i.e., considering stable sampling subsets of the $I$ sensor nodes and applying the power allocation for critically sampling to each sub-WSNs. One option then is to impose a round-robin (serial) scheduling where the sub-WSNs are alternately used for reconstruction. This reduces the energy consumption because inactive sensor nodes can temporarily be switched off while it is assured that over time all sensor nodes are used in the reconstruction process. The second option is to use a parallel scheduling, i.e., the sub-WSNs simultaneously contribute to the reconstruction by allocating the power to the sensor nodes of the sub-WSN according to (4.12) and by averaging the results obtained by the different sub-WSNs (see [4]). That way, all sensor nodes are active all the time, achieving a better reconstruction quality at the expense of larger energy consumption. Serial and parallel schedules could as well be combined to achieve a trade-off between energy consumption and reconstruction quality. 


\section{A Optimization Problem Revisited}

The matrix $\mathbf{G}_{\mathbf{g}}$ is positive definite and can hence be factorized as

$$
\mathbf{G}_{\mathrm{g}}=\mathbf{G}_{\mathrm{g}}^{1 / 2} \mathbf{G}_{\mathrm{g}}^{1 / 2}
$$

where $\mathbf{G}_{\mathrm{g}}^{1 / 2}$ is again positive definite and hence invertible [64]. The MSE of the reconstructed field in (4.8) can thus be rewritten as

$$
\begin{aligned}
\varepsilon & =\operatorname{tr}\left\{\left(\mathbf{G}^{T} \mathbf{D} \mathbf{G}\right)^{-1} \mathbf{G}_{\mathbf{g}}\right\}=\operatorname{tr}\left\{\left(\mathbf{G}^{T} \mathbf{D} \mathbf{G}\right)^{-1} \mathbf{G}_{\mathbf{g}}^{1 / 2} \mathbf{G}_{\mathbf{g}}^{1 / 2}\right\} \\
& =\operatorname{tr}\left\{\mathbf{G}_{\mathbf{g}}^{1 / 2}\left(\mathbf{G}^{T} \mathbf{D} \mathbf{G}\right)^{-1} \mathbf{G}_{\mathbf{g}}^{1 / 2}\right\}=\operatorname{tr}\left\{\left(\mathbf{G}_{\mathbf{g}}^{-1 / 2} \mathbf{G}^{T} \mathbf{D} \mathbf{G} \mathbf{G}_{\mathbf{g}}^{-1 / 2}\right)^{-1}\right\} \\
& =\operatorname{tr}\left\{\left(\sum_{i=1}^{I} d_{i} \mathbf{G}_{\mathbf{g}}^{-1 / 2} \mathbf{g}_{i} \mathbf{g}_{i}^{T} \mathbf{G}_{\mathbf{g}}^{-1 / 2}\right)^{-1}\right\}=\operatorname{tr}\left\{\left(\sum_{i=1}^{I} d_{i} \mathbf{u}_{i} \mathbf{u}_{i}^{T}\right)^{-1}\right\}
\end{aligned}
$$

where $\mathbf{g}_{i}$ denotes the columns of $\mathbf{G}^{T}$ and $\mathbf{u}_{i}=\mathbf{G}_{\mathbf{g}}^{-1 / 2} \mathbf{g}_{i}$. Further using the one-to-one correspondence

$$
p_{i}=\frac{\sigma_{w}^{2} d_{i}}{\gamma_{i}\left(1-\sigma_{v_{i}}^{2} d_{i}\right)}
$$

and introducing the slack variable

$$
\mathbf{s}=\left(s_{1} \ldots s_{J}\right)^{T}
$$

the problem in (4.9) can be cast as [10]

$$
\begin{array}{ll}
\underset{\mathbf{s}, \mathbf{d}}{\operatorname{minimize}} & \mathbf{1}^{\mathbf{T}} \mathbf{s} \\
\text { subject to } & {\left[\begin{array}{cc}
\sum_{i}^{I} d_{i} \mathbf{u}_{i} \mathbf{u}_{i}^{T} & \mathbf{e}_{p} \\
\mathbf{e}_{p}^{T} & s_{p}
\end{array}\right] \succeq 0, \quad p=1, \ldots, J} \\
& \mathbf{d} \succeq 0, \\
& \sigma_{w}^{2} \sum_{i=1}^{I} \frac{d_{i}\left(\sigma_{h}^{2}+\sigma_{v_{i}}^{2}\right)}{\gamma_{i}\left(1-\sigma_{v_{i}}^{2} d_{i}\right)} \leq P_{\mathrm{T}}, \\
& d_{i}-\frac{1}{\sigma_{v_{i}}^{2}} \leq 0 .
\end{array}
$$

Here $\mathbf{e}_{p}$ is the $p$ th unit vector and the last inequality ensures $p_{i} \geq 0$. 


\section{5}

\section{Conclusions and Outlook}

$\mathrm{W}$

E finally summarize the most important aspects and results of our work, present some conclusions, and provide ideas and suggestions for further research.

In this dissertation we used shift-invariant spaces to model physical fields and presented a scheme for field reconstruction from irregular samples based on this model. Our scheme allows the reconstruction of bandlimited fields as well as non-bandlimited fields, depending on the generator functions used. We used generator functions of compact support, in particular Basis-splines (B-splines), that provide excellent interpolation properties and allow us to reduce the computational overhead of the reconstruction. Moreover, they enable to partition the sensor nodes of the Wireless Sensor Network (WSN) into clusters that reconstruct smaller parts of the field independently. We introduced hybrid shift-invariant spaces that allow (compared to conventional shift-invariant spaces) better adaption to local smoothness properties of the field to be reconstructed by using different orders of B-splines within the various sensor node clusters. For the reconstruction of time-varying fields, we used iterative solvers in the field reconstruction scheme to further reduce the computational complexity. For clusters with corresponding overlapping subregions, we showed that averaging of the results of the field reconstruction leads to better reconstruction quality. To study the impact of imperfect sensor locations on the field reconstruction quality we analytically derived the influence of sensor localization errors on the Mean Square Error (MSE) of the reconstructed field and compared it for several B-spline orders.

For the system model used in the field reconstruction scheme we introduced an Amplify-and-Forward (AF) scheme for the transmission of sensor node measurements to the fusion center. Based on that model, we derived the optimal power allocation for minimal MSE and minimal transmit sum power, respectively. In both cases the problem of power allocation can be posed as convex optimization problems which can be solved numerically. For the special case of critical sampling we derived closed-form solutions for these convex problems. In numerical simulations we compared the performance of the optimal power allocation schemes, the optimal schemes for the case of critical sampling, and uniform power allocation. 


\subsection{Conclusions}

Based on the results in the previous chapters, we can draw the following conclusions.

- The field reconstruction scheme based on shift-invariant spaces introduced in this dissertation allows reconstruction of both bandlimited and non-bandlimited functions while other schemes assume or require either (strict) band limitation or significant oversampling of the function to be reconstructed. The field model can be adapted to the underlying function by smartly choosing the generator functions that span the shift-invariant space.

- The use of generator functions with compact support, in our case B-splines, renders the reconstruction problem sparse, hence leading to a reduced computational complexity of the reconstruction scheme.

- Compact support of the generator functions allows flexible partitioning of the WSN into sensor node clusters that can in parallel and independently reconstruct smaller parts of the field. This leads to a reduced communication overhead and a more robust reconstruction.

- Hybrid shift-invariant spaces are a generalization of conventional shift-invariant spaces. In a clustered WSN, they can be used to better adapt to local smoothness properties of the field to be reconstructed and yields better reconstruction quality.

- The MSE of the reconstructed field depends on the correlation matrix of the estimation error of the field coefficients and the Gramian of the generator functions. For an AF transmission strategy the correlation matrix of the estimation error of the field coefficients depends on the sensor node positions, the sensor measurement noise, the receiver noise, the transmission channel coefficients, and the power scaling factors.

- We analyzed the influence of sensor localization errors on the MSE of the reconstructed field and compared it for different orders of B-splines and for bandlimited reconstruction, i.e., a sinc-type generator function. Reconstruction using B-splines is less sensitive to errors in the sensor position estimates than reconstruction using the sinc-type generator function. For zero-order B-splines, there is literally no influence on the reconstruction quality, as long as the magnitude of the localization error is less than half the support of the zero-order B-spline. Among all B-spline generator functions, the order $N=1$ shows the maximal influence on the localization error which decreases monotonically for increasing B-spline order. The reason for that behaviour is the curvature of the particular generator functions around the zero point.

- For time-varying fields, it is advantageous to use iterative algorithms to solve the system of linear equations underlying the field reconstruction scheme. Using the results of previous time-slots for initialization, iterative solvers allow vast savings in the computational complexity of our field reconstruction scheme.

- Within overlapping subregions corresponding to neighboring sensor node clusters, the quality of the reconstructed field can be enhanced by appropriately averaging the estimates for the field coefficients. 
- The optimal sensor node power allocation for an AF transmission scheme depends on the sensor measurement noise, the receiver noise, the transmission channel coefficients, the power scaling factors, and the sensor node positions. This position reflects the sensor node's importance to render the sampling set stable.

- For our field reconstruction scheme, the constrained MSE-optimal and the transmit sum-power optimal power allocation schemes for AF transmission are convex problems that have no closed-form solution, but can be efficiently solved numerically.

- For the special case of critical sampling, the convex problems for the constrained MSE-optimal and the transmit sum-power optimal power allocation schemes for AF transmission allow to calculate closedform solutions. These solutions can also be used for the non-critical sampling case, where they provide good approximations for the optimal solution, but the quality of the approximation decays with increasing number of sensor nodes.

\subsection{Outlook}

There remain several open points and possible extensions for further research. We provide a brief and by no means exhaustive discussion in the following.

- Optimal B-spline order: In order to adapt the field model to the physical field to be reconstructed, the order of the B-splines used within each sensor node cluster has to be selected carefully. We only compared different orders and empirically determined the optimal one. Our field reconstruction scheme lacks an algorithm for the automatic B-spline order selection based on the properties of the underlying field. Such a scheme could be based on using a large fraction (e.g., 95 percent) of the sensor nodes for the reconstruction and the rest for assessment of the reconstruction quality.

- Extensions of the field model: It is possible to extend the field model in several ways. For example, a spatial scaling of the generator functions, e.g., inversely proportional to the sensor node density, could be incorporated. Moreover, the shift-invariant space model could not only be used for the spatial domain but also be extended to the time domain to appropriately model the temporal field evolution.

- Adaptive clustering algorithm: We assumed appropriate partitioning of the WSN into clusters that perform field reconstruction independently, where we performed clustering only manually. A suitable, self-organizing algorithm for clustering has to be robust against sensor node failures, has to balance communication and computation load among the sensor nodes, and, most importantly, establish stable sets of sampling within the clusters as this is necessary for successful reconstruction. Existing approaches for WSN presented in [16] could be modified to incorporate the requirement for a stable sampling set.

- Distributed computation of coefficients: Our scheme allows distribution of the field reconstruction problem to the sensor nodes only on the basis of clusters, where the field is reconstructed by the cluster head of each cluster. For a complete distribution of the reconstruction task among all sensor nodes of the 
WSN, message-passing algorithms, such as belief propagation or gossip algorithms, could be properly adapted for the computation of the field coefficients.

- Trade-off between number of sensor nodes and number of temporal snapshots: The idea for this trade-off is motivated by the bit-conservation principle presented in [32]. The possibility to trade off between the Analog-to-Digital (A/D) quantization precision per sensor node sample and the average sampling rate allows to flexibly assign a certain bit budget per Nyquist-period to either one of these domains. With respect to the reconstruction quality, a similar trade-off can be expected between the number of sensor nodes and the number of temporal snapshots, i.e., between the spatial and temporal sampling density.

- Transmission model: In addition to the analog AF transmission model in Chapter 4 the resource allocation problem for a Compress-and-Forward (CF) transmission model can be derived. In a CF model, the sensor node measurement data are quantized and digital messages are transmitted to the fusion center that uses these messages for field reconstruction. A resource allocation scheme based on this digital transmission model would assign a bit budget for the messages transmitted by each sensor node as a function its measurement quality and location in the WSN.

- Algorithms and performance limits: Motivated by [65], one could investigate further algorithms and their performance limits for cooperative multi-terminal inference, e.g., two terminals measure distributed but correlated data and cooperate to transmit information to a fusion center which detects or estimates parameters. Note that the setup for cooperative multi-terminal inference is different to the setups treated in [65], in that we neither aim to recover data symbols like in classical cooperative communications nor do we want to reproduce source signals like in distributed source coding. Moreover, it is different from classical multi-terminal inference where cooperation seems not to have been considered up to now.

- Secrecy aspects: In a WSN without a fusion center, utilizing completely distributed algorithms, we might be interested in the protection of secret information. Two correlated parts of the data may then be coded in a way that allows transmission of the one part to the receiver within a prescribed distortion tolerance while keeping the other part as secret as possible from the receiver or wiretappers. For this purpose, the ideas of [66], where this source coding problem with multiple constraints was studied for a one-way communication system, can be extended to the multi-terminal communication case prevailing in WSN. 


$\begin{array}{ll}\text { 1-D } & \text { One-Dimensional } \\ \text { 2-D } & \text { Two-Dimensional } \\ \text { A/D } & \text { Analog-to-Digital } \\ \text { AF } & \text { Amplify-and-Forward } \\ \text { AOA } & \text { Angle of Arrival } \\ \text { ASIC } & \text { Application Specific Integrated Circuit } \\ \text { B-spline } & \text { Basis-spline } \\ \text { BLUE } & \text { Best Linear Unbiased Estimator } \\ \text { CDMA } & \text { Code Division Multiple Access } \\ \text { CF } & \text { Compress-and-Forward } \\ \text { CPU } & \text { Central Processing Unit } \\ \text { CRLB } & \text { Cramér-Rao Lower Bound } \\ \text { DES } & \text { Decentralized Estimation Scheme } \\ \text { ESPRIT } & \text { Estimation of Signal Parameters by Rotational Invariance Techniques } \\ \text { FDMA } & \text { Frequency Division Multiple Access } \\ \text { FPGA } & \text { Field Programmable Gate Array } \\ \text { GMRES } & \text { Generalized Minimal RESidual method } \\ \text { GNSS } & \text { Global Navigation Satellite System } \\ \text { GPS } & \text { Global Positioning System } \\ \text { KKT } & \text { Karush-Kuhn-Tucker } \\ \text { LMMSE } & \text { Linear Minimum Mean Square Error } \\ \text { LOS } & \text { Line-Of-Sight } \\ \text { LS } & \text { Least Squares } \\ \text { MAC } & \text { Multiple-Access Channel } \\ \text { MAP } & \text { Maximum A Posteriori } \\ \text { ML } & \text { Maximum Likelihood } \\ \text { MMSE } & \text { Mean Square Error } \\ \text { MSE } & \end{array}$




$\begin{array}{ll}\text { MUSIC } & \text { Multiple Signal Classification } \\ \text { MVU } & \text { Minimum Variance Unbiased } \\ \text { NLOS } & \text { Non-Line-Of-Sight } \\ \text { pdf } & \text { Probability Density Function } \\ \text { QAM } & \text { Quadrature Amplitude Modulation } \\ \text { RSS } & \text { Received Signal Strength } \\ \text { SNR } & \text { Signal-to-Noise Ratio } \\ \text { TDMA } & \text { Time Division Multiple Access } \\ \text { TDOA } & \text { Time Difference Of Arrival } \\ \text { TOA } & \text { Time Of Arrival } \\ \text { UWB } & \text { Ultra Wide Band } \\ \text { WLAN } & \text { Wireless Local Area Network } \\ \text { WLS } & \text { Weighted Least Squares } \\ \text { WSN } & \text { Wireless Sensor Network }\end{array}$


The following notation is used throughout the thesis. Scalars are denoted by italic letters, e.g., $x$ or $X$. Boldface letters are used for vectors and matrices, e.g., $\mathbf{x}$ and $\mathbf{X}$, respectively. Moreover, $[\mathbf{x}]_{m}$ denotes the $m$ th element of the vector $\mathbf{x}$ and $[\mathbf{X}]_{m, l}$ denotes the element in row $m$ and column $l$ of the matrix $\mathbf{X}$. Calligraphic upper-case letters, e.g., $\mathcal{A}$, refer to sets. Furthermore, no notational distinction is made between random variables and their realizations. Additional notational conventions are:

$\begin{array}{ll}\mathbf{I} & \text { Identity matrix, unit matrix } \\ g(x, y) & \text { Generator function } \\ g_{\mathrm{BL}}(x, y) & \text { Bandlimited sinc-type generator function } \\ b_{N}(x, y) & \text { Two-Dimensional (2-D) Order N B-spline } \\ \tilde{b}_{N}(x) & \text { One-Dimensional (1-D) Order N B-spline } \\ h(x, y ; t) & \text { Physical field } \\ \mathcal{A} & \text { Spatial region } \\ \mathcal{A}_{m} & \text { Spatial subregion } \\ \mathcal{C}_{m} & \text { Sensor node cluster } \\ c_{k, l} & \text { Field coefficient } \\ \hat{c}_{k, l} & \text { Field coefficient estimate } \\ I & \text { Number of sensors } \\ M & \text { Number of subregions/clusters } \\ \mathcal{S} & \text { Support } \\ I_{m}=\left|\mathcal{C}_{m}\right| & \text { Number of sensors in cluster } \mathcal{C}_{m} \\ J_{m} & \text { Number of coefficients } c_{k, l} \text { in } \mathcal{A}_{m} \\ \mathbb{Z} & \text { Set of integer numbers } \\ \mathbb{A}=\mathbb{Z}^{2} \cap\left(\mathcal{A}_{m}+\mathcal{S}\right) & \text { Set of integer numbers in region } \mathcal{A} \\ \mathbb{R} & \text { Set of real numbers } \\ \mathbb{R}+ & \text { Set of positive real numbers } \\ (x, y) & \text { Spatial Coordinates } \\ \left(x_{i}, y_{i}\right) & \text { Position of sensor node } i \\ t & \text { Time (continuous case) } \\ & \end{array}$




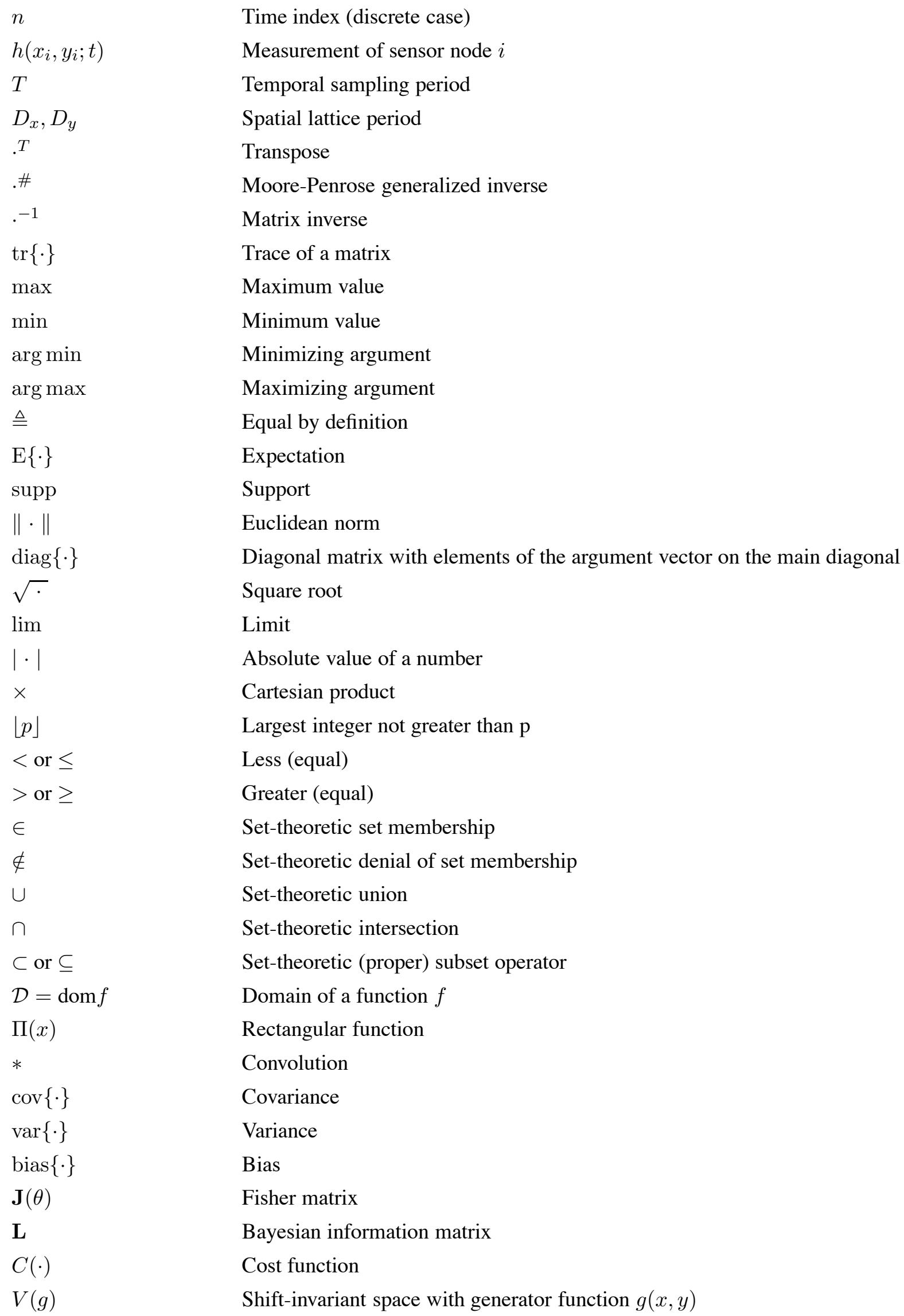

$n$

$h\left(x_{i}, y_{i} ; t\right)$

T

$D_{x}, D_{y}$

.T

.\#

.-1

$\operatorname{tr}\{\cdot\}$

$\max$

$\min$

$\arg \min$

$\arg \max$

$\triangleq$

$\mathrm{E}\{\cdot\}$

supp

\|.

$\operatorname{diag}\{\cdot\}$

$\sqrt{\cdot}$

$\lim$

$|\cdot|$

$\times$

$\lfloor p\rfloor$

$<$ or $\leq$

$>$ or $\geq$

$\epsilon$

$\notin$

$\cup$

$\cap$

$\subset$ or $\subseteq$

$\mathcal{D}=\operatorname{dom} f$

$\Pi(x)$

$*$

$\operatorname{cov}\{\cdot\}$

$\operatorname{var}\{\cdot\}$

$\operatorname{bias}\{\cdot\}$

$\mathbf{J}(\theta)$

L

$C(\cdot)$

$V(g)$

Time index (discrete case)

Measurement of sensor node $i$

Temporal sampling period

Spatial lattice period

Transpose

Moore-Penrose generalized inverse

Matrix inverse

Trace of a matrix

Maximum value

Minimum value

Minimizing argument

Maximizing argument

Equal by definition

Expectation

Support

Euclidean norm

Diagonal matrix with elements of the argument vector on the main diagonal

Square root

Limit

Absolute value of a number

Cartesian product

Largest integer not greater than $\mathrm{p}$

Less (equal)

Greater (equal)

Set-theoretic set membership

Set-theoretic denial of set membership

Set-theoretic union

Set-theoretic intersection

Set-theoretic (proper) subset operator

Domain of a function $f$

Rectangular function

Convolution

Covariance

Variance

Bias

Fisher matrix

Bayesian information matrix

Cost function

Shift-invariant space with generator function $g(x, y)$ 
$\lambda_{x}, \lambda_{y}$

$N$

$N_{\max }$
Sensor node position jitter

B-spline order

Maximum B-spline order 


\section{Bibliography}

[1] G. Reise and G. Matz, "Distributed sampling and reconstruction of non-bandlimited fields in sensor networks based on shift invariant spaces," in Proc. ICASSP 2009, (Taipeh, Taiwan), pp. 2061-2064, Apr. 2009.

[2] G. Reise and G. Matz, "Clustered wireless sensor networks for robust distributed field reconstruction based on hybrid shift-invariant spaces," in Proc. SPAWC 2009, (Perugia, Italy), pp. 66-70, June 2009.

[3] G. Reise and G. Matz, "Reconstruction of time-varying fields in wireless sensor networks using shiftinvariant spaces: iterative algorithms and impact of sensor localization errors," in Proc. SPAWC 2010, (Marrakech, Morocco), pp. 1-5, June 2010. Proc. SPAWC 2010.

[4] G. Reise, G. Matz, and K. Gröchenig, "Distributed field reconstruction in wireless sensor networks based on hybrid shift-invariant spaces," submitted to IEEE Trans. Signal Process., 2011.

[5] G. Reise, J. Matamoros, C. Antón-Haro, and G. Matz, "MSE-optimal power allocation in wireless sensor networks for field reconstruction based on shift-invariant spaces," in Proc. 45th Asilomar Conf. Signals, Systems, Computers, (Pacific Grove, CA, USA), Nov. 2011.

[6] G. Reise and G. Matz, "Optimal allocation of transmit power in wireless sensor networks for field reconstruction based on shift-invariant spaces.” submitted to ICASSP 2012, Mar. 2012.

[7] G. Reise, J. Matamoros, C. Antón-Haro, and G. Matz, "Optimal power allocation in wireless sensor networks for field reconstruction based on shift-invariant spaces," to be submitted to IEEE Trans. Signal Process.

[8] A. Aldroubi and K. Gröchenig, "Nonuniform sampling and reconstruction in shift-invariant spaces," SIAM Review, vol. 43, no. 4, pp. 585-620, 2001.

[9] K. Gröchenig and H. Schwab, "Fast local reconstruction methods for nonuniform sampling in shiftinvariant spaces," SIAM J. Matrix Anal. Appl., vol. 24, pp. 899-913, April 2003.

[10] S. Boyd and L. Vandenberghe, Convex Optimization. Cambridge University Press, 2004. 
[11] H. Labiod, ed., Wireless Ad-Hoc and Sensor Networks. Wiley \& Sons, 2008.

[12] I. F. Akyildiz and M. C. Vuran, Wireless Sensor Networks. Wiley \& Sons, 2010.

[13] I. F. Akyildiz, W. Su, Y. Sankarasubramaniam, and E. Cayirci, "Wireless sensor networks: A survey," Computer Networks, vol. 38, pp. 393 - 422, August 2002.

[14] H. Karl and A. Willig, Protocols and Architectures for Wireless Sensor Networks. Wiley \& Sons, 2005.

[15] J. N. Al-Karaki and A. E. Kamal, "Routing techniques in wireless sensor networks: A survey," IEEE Wireless Communications, vol. 11, pp. 6-28, Dec. 2004.

[16] A. A. Abbasi and M. Younis, "A survey on clustering algorithms for wireless sensor networks," Computer Communications, vol. 30, pp. 2828-2841, Oct. 2007.

[17] O. Younis, M. Krunz, and S. Ramasubramanian, "Node clustering in wireless sensor networks: Recent developments and deployment challenges," IEEE Netw., vol. 20, pp. 20-25, May-June 2006.

[18] G. Mao, B. Fidan, and B. D. O. Anderson, "Wireless sensor network localization techniques," Computer Networks: The International Journal of Computer and Telecommunications Networking, vol. 51, no. 10, pp. 2529-2553, 2007.

[19] L. Hu and D. Evans, "Localization for mobile sensor networks," in Proc. Tenth Annual International Conference on Mobile Computing and Networking, 2004.

[20] J. Hightower and G. Borriello, "A survey and taxonomy of location systems for ubiquitous computing," Tech. Rep. UW-CSE-01-08-03, University of Washington, Computer Science and Engineering, 2001.

[21] S. Haykin and K. J. Ray Liu, eds., Handbook on Array Processing and Sensor Networks. Wiley \& Sons, 2009.

[22] K. Langendoen and N. Reijers, "Distributed localization in wireless sensor networks: A quantitative comparison," Computer Networks: The International Journal of Computer and Telecommunications Networking, vol. 43, no. 4, pp. 499-518, 2003.

[23] J. Hightower and G. Borriello, "Location systems for ubiquitous computing," IEEE Computer, vol. 34, pp. 57-66, Aug. 2001.

[24] J. Hightower and G. Borriello, "Location sensing techniques," Tech. Rep. UW-CSE-01-07-01, University of Washington, Computer Science and Engineering, 2001.

[25] S. Gezici, Z. Tian, G. B. Giannakis, H. Kobayashi, A. F. Molisch, H. V. Poor, and Z. Sahinoglu, "Localization via ulta-wideband radios," IEEE Signal Processing Magazine, vol. 22, pp. 70-84, July 2005.

[26] Z. Sahinoglu, S. Gezici, and I. Güvenc, Ultra-wideband Positioning Systems: Theoretical Limits, Ranging Algorithms, and Protocols. Cambridge University Press, 2008. 
[27] Y.-C. Wu, Q. Chadhari, and E. Serpedin, "Clock synchronization of wireless sensor networks," IEEE Signal Processing Magazine, vol. 28, pp. 124-138, Jan. 2011.

[28] G. Sun, J. Chen, and W. Guo, "Signal processing techniques in network-aided positioning," IEEE Signal Processing Magazine, vol. 22, pp. 12-23, July 2005.

[29] S. M. Kay, Fundamentals of Statistical Signal Processing: Estimation Theory. Prentice Hall, Inc., 1993.

[30] H. L. Van Trees, Detection, Estimation, and Modulation Theory. Part I. John Wiley \& Sons, 1968.

[31] J. C. Dattorro, Convex Optimization \& Euclidean Distance Geometry. Meboo Publishing, 2005.

[32] P. Ishwar, A. Kumar, and K. Ramchandran, "Distributed sampling in dense sensor networks: a 'bitconservation principle"," in Proc. IEEE Int. Symp. Information Processing in Sensor Networks, (Palo Alto, CA), pp. 17-31, Apr. 2003.

[33] A. Nordio, C. F. Chiasserini, and E. Viterbo, "Performance of linear field reconstruction techniques with noise and uncertain sensor locations," IEEE Trans. Signal Processing, vol. 56, pp. 3535-3547, Aug. 2008.

[34] A. B. Gershman and V. I. Turchin, "Nonwave field processing using sensor array approach," Signal Processing, vol. 44, pp. 197-210, 1995.

[35] A. Kumar, P. Ishwar, and K. Ramchandran, "On distributed sampling of smooth non-bandlimited fields," in Proc. IEEE Int. Symp. Information Processing in Sensor Networks, (Berkeley, CA), pp. 89-98, Apr. 2004.

[36] F. S. Cattivelli, C. G. Lopes, and A. H. Sayed, "Diffusion recursive least-squares for distributed estimation over adaptive networks,” IEEE Trans. Signal Process., vol. 56, pp. 1865-1877, Jan. 2008.

[37] S. Boyd, A. Ghosh, B. Prabhakar, and D. Shah, "Randomized gossip algorithms," IEEE Trans. Inf. Theory, vol. 52, pp. 2508-2530, June 2006.

[38] C. C. Moallemi and B. V. Roy, “Consensus propagation," IEEE Trans. Inf. Theory, vol. 52, pp. 4753-4766, Nov. 2006.

[39] R. Olfati-Saber, J. Fax, and R. Murray, "Consensus and cooperation in networked multi-agent systems," Proc. IEEE, vol. 95, pp. 215-233, Jan. 2007.

[40] F. A. Marvasti, ed., Nonuniform Sampling: Theory and Practice. New York, USA: Kluwer Academic Publishers, 2001.

[41] Q. Sun, "Frames in spaces with finite rate of innocation," Advances in Computational Mathematics, vol. 28, no. 301-329, 2008.

[42] O. Christensen, An introduction to Frames and Riesz bases. Birkhäuser, 2003. 
[43] J. H. Ahlberg, E. N. Nilson, and J. L. Walsh, The Theory of Splines and Their Application, vol. 38. Academic Press Inc., 1967.

[44] C. de Boor, A Practical Guide to Splines. Springer Verlag, 2 ed., 2001.

[45] G. Golub and C. V. Loan, Matrix Computations. Johns Hopkins University Press, 3 ed., 1996.

[46] A. Edelman, Eigenvalues and Condition Numbers of Random Matrices. PhD thesis, Massachusetts Institute of Technology, Cambridge (MA), 1989.

[47] I. S. Duff, A. M. Erisman, and J. K. Reid, Direct Methods for Sparse Matrices. Oxford University Press, England, 1986.

[48] N. I. M. Gould, J. A. Scott, and Y. Hu, "A numerical evaluation of sparse direct solvers for the solution of large sparse symmetric linear systems of equations," ACM Trans. Math. Softw., vol. 33, June 2007.

[49] Y. Saad and M. Schultz, "GMRES: A generalized minimal residual algorithm for solving nonsymmetric linear systems.," SIAM J. Sci. Stat. Comput., vol. 7, pp. 856-869, July 1986.

[50] C. C. Paige and M. A. Saunders, "LSQR: An algorithm for sparse linear equations and sparse least squares," ACM Trans. on Mathematical Software, vol. 8, pp. 43-71, Mar. 1982.

[51] A. Aldroubi and K. Gröchenig, "Beurling-landau-type theorems for non-uniform sampling in shift invariant spline spaces," Journal of Fourier Analysis and Applications, vol. 6, pp. 93-103, Jan. 2000.

[52] A. Aldroubi and H. G. Feichtinger, "Exact iterative reconstruction algorithm for multivariate irregularly sampled functions in spline-like spaces: the $L^{p}$-theory," Proc. Amer. Math. Soc., vol. 126, no. 9, pp. $2677-$ 2686, 1998.

[53] R. F. Bass and K. Gröchenig, "Random sampling of multivariate trigonometric polynomials," SIAM J. Matrix Anal. Appl., vol. 36, no. 3, pp. 779-795 (electronic), 2004/05.

[54] M. G. Cox, "The numerical evaluation of B-splines," J. Inst. Maths. Applics., vol. 10, pp. 134-149, August 1972.

[55] M. Gastpar and M. Vetterli, "Source-channel communication in sensor networks," in Information Processing in Sensor Networks (F. Zhao and L. Guibas, eds.), vol. 2634 of Lecture Notes in Computer Science, pp. 553-553, Springer Berlin / Heidelberg, 2003.

[56] T. M. Cover and J. A. Thomas, Elements of Information Theory. Wiley \& Sons, 2 ed., 2006.

[57] J.-J. Xiao and Z.-Q. Luo, "Decentralized estimation in an inhomogeneous sensing environment," IEEE Trans. Inf. Theory, vol. 51, pp. 3564-3575, Oct. 2005.

[58] J.-J. Xiao, S. Cui, Z.-Q. Luo, and A. Goldsmith, "Power scheduling of universal decentralized estimation in sensor networks," IEEE Trans. Signal Process., vol. 54, pp. 413-422, Feb. 2006. 
[59] S. Cui, J.-J. Xiao, A. J. Goldsmith, Z.-Q. Luo, and H. V. Poor, "Energy-efficient joint estimation in sensor networks: Analog vs. digital," in Proc. ICASSP 2005, 2005.

[60] S. Cui, J.-J. Xiao, A. Goldsmith, Z.-Q. Luo, and H. V. Poor, "Estimation diversity and energy efficiency in distributed sensing," IEEE Trans. Signal Process., vol. 55, pp. 4683-4695, Sept. 2007.

[61] I. Bahceci and A. Khandani, "Linear estimation of correlated data in wireless sensor networks with optimum power allocation and analog modulation," IEEE Trans. Commun., vol. 56, pp. 1146-1156, July 2008.

[62] J. Fang and H. Li, "Power constrained distributed estimation with correlated sensor data," IEEE Trans. Signal Process., vol. 57, pp. 3292-3297, August 2009.

[63] G. Kramer, I. Marić, and R. D. Yates, "Cooperative communications," Found. Trends Netw., vol. 1, pp. 271-425, Aug. 2006.

[64] R. A. Horn and C. R. Johnson, Matrix Analysis. Cambridge University Press, 1990.

[65] T. S. Han and S.-I. Amari, "Statistical inference under multiterminal data compression," IEEE Trans. Inf. Theory, vol. 44, pp. 2300-2324, Oct. 1998.

[66] H. Yamamoto, "A source coding problem for sources with additional outputs to keep secret from receiver or wiretappers," IEEE Trans. Inf. Theory, vol. 29, pp. 918-923, Nov. 1983. 